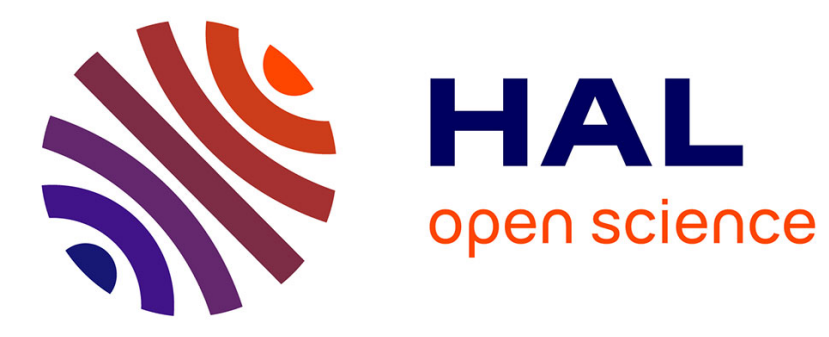

\title{
Reducing Inequalities among Unequals
}

Mathieu Faure, Nicolas Gravel

\section{To cite this version:}

Mathieu Faure, Nicolas Gravel. Reducing Inequalities among Unequals. International Economic Review, 2021, 62 (1), pp.357 - 404. 10.1111/iere.12490 . hal-03002498

\section{HAL Id: hal-03002498 \\ https://hal-amu.archives-ouvertes.fr/hal-03002498}

Submitted on 10 Feb 2021

HAL is a multi-disciplinary open access archive for the deposit and dissemination of scientific research documents, whether they are published or not. The documents may come from teaching and research institutions in France or abroad, or from public or private research centers.
L'archive ouverte pluridisciplinaire HAL, est destinée au dépôt et à la diffusion de documents scientifiques de niveau recherche, publiés ou non, émanant des établissements d'enseignement et de recherche français ou étrangers, des laboratoires publics ou privés. 


\title{
REDUCING INEQUALITIES AMONG UNEQUALS
}

\author{
By Mathieu Faure and Nicolas Gravel ${ }^{1}$ \\ Centre de Sciences Humaines (Delhi) \& Aix-Marseille University, CNRS, AMSE
}

\begin{abstract}
This article establishes an equivalence between four incomplete rankings of distributions of income among agents who are vertically differentiated with respect to some nonincome characteristic (health, household size, etc.). The first ranking is the possibility of going from one distribution to the other by a finite sequence of income transfers from richer and more highly ranked agents to poorer and less highly ranked ones. The second ranking is the unanimity among utilitarian planners who assume that agents' marginal utility of income is decreasing with respect to both income and the source of vertical differentiation. The third ranking is the Bourguignon (Journal of Econometrics, 42 (1989), 67-80) Ordered Poverty Gap dominance criterion. The fourth ranking is a new dominance criterion based on cumulative lowest incomes.
\end{abstract}

\section{INTRODUCTION}

When can a distribution of income among a group of homogeneous agents be considered more equal than another? An important achievement of the modern theory of inequality measurement is the demonstration made by Hardy et al. (1952) —and popularized among economists by Dasgupta et al. (1973), Kolm (1969), Sen (1973), and Fields and Fei (1978)_ that the following four answers to this question are equivalent.

(1) $A$ is more equal than $B$ if it can be obtained from $B$ by means of a finite sequence of bilateral Pigou-Dalton transfers.

(2) $A$ is more equal than $B$ if all utilitarian ethical observers who assume that individuals convert income into well-being by the same concave utility function so agree.

(3) $A$ is more equal than $B$ if poverty, as measured by the poverty gap, is lower in $A$ than in $B$ for every definition of the poverty line.

(4) $A$ is more equal than $B$ if the $k$ poorest agents have a larger cumulated income in $A$ than in $B$ whatever $k$ is (i.e., if the distribution of income in $A$ Lorenz dominates that in $B$ ).

Any of these answers provides an incomplete, but yet very robust, answer to the basic question. The first answer identifies an elementary transformation of the distribution that intuitively captures in a crisp fashion the very notion of inequality reduction-Pigou-Dalton here-that is sought. The second answer links inequality measurement to a set of explicit normative principles and seeks consensus among them. Although answer (2) is formulated in terms of the ethically contentious doctrine of utilitarianism, it can actually be shown (see,

\footnotetext{
${ }^{1}$ With the usual disclaiming qualification, we are indebted to Sebastian Bervoets, Yann Bramoullé, Alain Chateauneuf, and, especially, Patrick Moyes for many insightful comments and discussions. We have also received extremely valuable comments and suggestions from Masaki Aoyagi and three referees from this journal. Finally, we gratefully acknowledge the financial support of the French Agence Nationale de la Recherche (ANR) through four contracts: Measurement of Ordinal and Multidimensional Inequalities (ANR-16-CE41-0005), Challenging Inequalities: A Indo-European perspective (ANR-18-EQUI-0003), Communication and Information in Games on Networks (ANR-15-CE38-0007), and The European University of Research AMSE (ANR-17-EURE-020). Please address correspondence to: Nicolas Gravel, Centre de Sciences Humaines, 2, Dr APJ Abdul Kalam Road, 110011 Delhi, India; and Aix-Marseille University, CNRS, AMSE. Phone: +911130410090. E-mail: nicolas.gravel@univ-amu.fr.
} 
e.g., Gravel and Moyes, 2013) to hold for the much larger class of aggregations of concave utilities by increasing and utility-inequality-averse ethical observers. Moreover, even the welfarist or utilitarian interpretation underlying answer (2) is not required. The utility function can also be interpreted as reflecting the "social value" attached by the ethical observer to the income received by the agent. This social value may not be related to the agent's personal welfare or happiness. Finally, the third and fourth answers provide empirically implementable tests - poverty gap or Lorenz dominance-to determine whether or not one distribution is more equal than another. Comparing income distributions by means of Lorenz dominance has become a routine practice followed by thousands of researchers all over the world. Many sophisticated inference techniques-see, for example, Anderson (1996), Beach and Davidson (1983), Bishop et al. (1989a), Bishop and Formby (1999), and Davidson and Duclos (2000) - have also been proposed to assess the robustness of Lorenz dominance or stochastic dominance comparisons when applied to samples instead of the whole population of interest. Moreover, despite their possible incompleteness, these criteria have been shown in empirical applications - see for example Bishop et al. (1989b), Duclos et al. (2006), Gravel et al. (2009), or Gravel and Mukhopadhyay (2010)—-to rank conclusively a significant fraction of all the possible pairs of distributions. When such conclusive rankings cannot be obtained, and the Lorenz curves associated to the two distributions cross, it is common to compare distributions using (much) more ethically demanding inequality indices. In such a case, the requirement that the ranking provided by the inequality indices be compatible with any of those four answers is considered to be very important (see, e.g., Foster, 1985).

Remarkable and foundational to inequality measurement as it is, this equivalence only concerns distributions of income, or any other cardinally measurable attribute, between otherwise perfectly homogeneous agents. Yet, income is not the only ethically relevant source of differentiation between economic agents. If these agents are collectivities such as households or jurisdictions, they differ not only by their total income but also by the number of members among whom the income must be shared. If the agents are individuals, they may also differ by nonincome characteristics such as age, health, education, or effort. What does "reducing inequalities" in one characteristic mean when applied to agents who are differentiated with respect to another characteristic? In short, how can one define reducing inequality among unequals? This is the basic question addressed in this article.

Specifically, we establish an equivalence between four notions of inequality reduction among unequals, each of which being analogous in nature to one of the above four notions of inequality reduction among equals. The elementary transformation that we propose to capture inequality reduction among unequals is like a Pigou-Dalton transfer, but with the stipulation that the donor must be both richer and more highly ranked than the receiver. Moreover, contrary to what is usually required in a Pigou-Dalton transfer-see however Atkinson (1987), Chakravarty and Muliere (2003), or Zheng (2007) for alternative formulations-we do not restrict the transfer to being lower than half the income difference between the giver and the receiver. The quantity transferred can be as large as the full income difference. The normative principles that we examine are those generated by comparisons of distributions by a utilitarian ethical observer who assumes that agents convert income into utility by the same function exhibiting a marginal utility of income that is decreasing with respect to both income and the source of vertical differentiation. The empirically implementable criterion that we consider is the Bourguignon (1989) Ordered Poverty Gap (OPG) dominance criterion. This criterion requires that poverty, measured by the income poverty gap, be smaller in the dominating distribution than in the dominated distribution for any collection of poverty lines that are decreasing with respect to the agent's vertical standing. We finally introduce a "cumulated lowest incomes" dominance criterion-which generalizes Lorenz dominance to our settingand prove its equivalence with OPG dominance.

This article can clearly be seen as a contribution to the multidimensional-in fact twodimensional-inequality measurement literature, which has emerged in the last 40 years or so. To the best of our knowledge, no contribution to this literature has succeeded in establishing 
a foundational equivalence between an empirically implementable criterion (such as Lorenz or poverty gap dominance), a welfarist (or otherwise) unanimity over a class of functions that transform the attributes into achievement and an elementary operation that captures in an intuitive way the nature of the equalization sought.

For instance, Atkinson and Bourguignon (1982) (and before them Hadar and Russell (1974)) show that first- and second-order multidimensional stochastic dominance imply utilitarian dominance over a class of individual utility functions that is specific to the order of dominance. They also suggest (without providing proof) that there could be an equivalence between their multidimensional stochastic dominance criteria and utilitarian unanimity over their class of individual utility functions. But they do not identify an elementary operation that can be implied by their criteria or that can imply them. Atkinson and Bourguignon (1987) propose a nice interpretation of one of the Atkinson and Bourguignon (1982) stochastic dominance criteria in the specific case of two attributes, one of which interpreted as an ordinal index of needs (such as household size). Yet, they do not identify the elementary operation that, when performed a finite number of times, would coincide with the criterion. It is in the very same setting that Bourguignon (1989) introduces his OPG criterion. Bourguignon (1989) also identifies the class of utility functions over which utilitarian unanimity is equivalent to his criterion. However, he does not identify the elementary operation that is equivalent to it.

Elementary transformations believed to lie behind the criteria proposed by Atkinson and Bourguignon (1982), Atkinson and Bourguignon (1987), and Bourguignon (1989) have been discussed by various authors, including Atkinson and Bourguignon (1982) themselves, Ebert (1997), Fleurbaey et al. (2003), and Moyes (2012). Yet, none of these papers demonstrates that performing these elementary operations a finite number of times is equivalent to the implementable criteria. In a related vein, Muller and Scarsini (2012) establish an equivalence between a class of elementary transformations-multidimensional transfers and correlationreducing permutations, to be discussed below-and utilitarian unanimity over the class of increasing and submodular utility functions. ${ }^{2}$ However, they do not provide an implementable test—such as Lorenz or poverty gap dominance-that coincides with either their elementary transformations or the utilitarian unanimity over their class of utility functions. Another attempt to propose an elementary transformation that would capture a plausible notion of inequality reduction in a multidimensional context has been made by Kolm (1977). This author defines equalization by the fact of transferring from one agent to another an identical fraction of both income and the source of vertical differentiation. Making sense of this notion of equalization obviously requires that this source of vertical differentiation be cardinally measurable. Although this measurability may be conceivable in some context (e.g., when the agents are workers vertically differentiated by their number of hours worked), it is less so in others (e.g., households differentiated by the number of their members). Kolm (1977) proves that obtaining one distribution from another by means of a finite sequence of such transformations is equivalent to having the two distributions ranked by all utilitarian ethical observers who evaluate the agents' well-being by means of the same concave utility function. However, Kolm (1977) does not identify an empirically implementable criterion that is equivalent to his notion of equalization.

Another approach to multidimensional equalization is followed by Koshevoy (1995) who suggests that distributions of several attributes be compared on the basis of the inclusion of their Lorenz zonotope. This Lorenz zonotope inclusion criterion is a clear generalization, to any number of dimensions, of the usual unidimensional Lorenz dominance. It is a (relatively) easy-to-check criterion that is applicable to any two distributions of several attributes. Koshevoy (1995) proves that performing a finite number of times the elementary transformation proposed by Kolm (1977) is a sufficient condition for obtaining Lorenz zonotope inclusion. However, this does not tell us much about the implicit equalization process embedded in this criterion because the converse statement does not hold.

\footnotetext{
${ }^{2}$ See, for example, Marinacci and Montrucchio (2005) for a definition of these properties.
} 
Progress toward establishing equivalence between an empirically implementable criterion, a utilitarian unanimity over a suitable class of individual utility functions and a finite sequence of elementary transformations has been made in two streams of the literature. One of them, initiated by Epstein and Tanny (1980) (see also Tchen, 1980), and significantly generalized by Decancq (2012), considers first-order stochastic dominance rankings of multivariate distributions in the context of decision making under uncertainty. In this setting, Decancq (2012) establishes an equivalence between first-order dominance among multivariate distributions with the same marginals and the possibility of going from the dominated to the dominating distribution by a finite sequence of Frechet rearrangements. By significantly generalizing results from Quirk and Saposnik (1962) and Levhari et al. (1975), Osterdal (2010) also establishes an equivalence between utilitarian unanimity over the class of all increasing utility functions, the possibility of going from one distribution to another by a finite sequence of improving mass transfers, and a specific first-order stochastic dominance test that is less discriminant than the usual multivariate one considered in Hadar and Russell (1974) and Atkinson and Bourguignon (1982). None of these results, however, sheds light on the meaning of reducing income inequality between heterogenous agents.

An attempt in this direction has been made by Gravel and Moyes (2012), who establish a form of equivalence between the three following answers to the question of when a distribution $A$ of income between vertically differentiated agents is normatively better than another distribution $B$ :

(a) When $A$ could be obtained from $B$ by performing a finite sequence of either PigouDalton transfers of income between agents of the same type or correlation-reducing permutations.

(b) When $A$ is considered better than $B$ by all utilitarian planners who assume that vertically differentiated agents convert income into well-being by the same utility function whose marginal utility of income is decreasing with respect to both income and the source of vertical differentiation.

(c) When $A$ dominates $B$ by the OPG criterion.

Answer (a) combines two elementary operations. The first is the standard Pigou-Dalton transfer performed between agents of the same "type." The second is a correlation-reducing income permutation between two agents, one of them being both richer and more highly ranked than the other. A correlation-reducing permutation is an operation closely related to the notion of Frechet rearrangement used by Decancq (2012); see also Tsui (1999), Atkinson and Bourguignon (1982), and Epstein and Tanny (1980). Answers (b) and (c) are of course those considered here. However, Gravel and Moyes (2012) have not succeeded in proving that answer (c) (or answer (b)) implies answer (a) (clearly answer (a) implies answer (b), which in turn implies answer (c)). What they prove is that if distribution $A$ dominates distribution $B$ for the OPG criterion, then it is possible to add dummy individuals-or phantoms-to both distributions $A$ and $B$ in such a way as to be able to go from phantom-augmented distribution $B$ to phantom-augmented distribution $A$.

In this article, we prove the equivalence between the above answers (b) and (c) and the possibility of going from dominated to dominating distributions by a finite sequence of elementary transfers of income from richer and more highly ranked agents to poorer and less highly ranked agents. We do so without resorting to phantoms. Our notion of transfer contains as particular cases both the correlation- reducing permutation and the within-type transfer considered in Gravel and Moyes (2012). An additional contribution of this article is to establish an equivalence between the OPG criterion and a Lorenz-like dominance criterion based on partial sums of incomes of the poorest agents. A difficulty involved in the latter criterion is identifying the poorest agents when these agents are differentiated by a nonincome characteristic. Our main result shows that this criterion is equivalent to the OPG criterion. We therefore view this article as providing what is to our knowledge the first theoretical foundation to the measurement of inequality among heterogeneous agents. 
The remainder of the article is organized as follows. In the next section, we introduce notations and provide definitions of the main criteria and elementary transformations considered. The main results are stated and discussed in Section 3. Section 4 indicates how the results of Section 3 extend to the case where the number of agents and/or the total income to be distributed vary across distributions, and Section 5 concludes.

\section{THE FORMAL SETTING}

2.1. Notations. We consider a finite population of $n$ agents who are vertically differentiated into $k$ categories or types, indexed by $h$. Agents in lower categories are assumed to be "more needy" ceteris paribus than agents in higher categories. These categories may refer to any nonpecuniary source of agents' differentiation, such as health, number of members, education level, labor effort. We do not assume at this stage (see however the generalization of the analysis in Section 4) that the ordering of the agents in terms of those categories reflects these agents' well-being. For example, a single adult may admittedly be considered less needy than a couple who earns the same income. However, this does not mean that the single adult is "happier" or "better off" than (either member of) this couple. We emphasize also that we do not require the source of vertical differentiation to be discrete. It could be continuous (as would, e.g., be the number of hours worked). However, for any finite population of agents of size $n$, the set of possible values taken by the nonpecuniary source of differentiation in that population will be finite. It is therefore not restrictive to view these different values as underlying the categories. In the current as well as the next sections, we consider the case where both the total number of agents and their distributions in the $k$ different categories is fixed. In this case, for any category $h$, we denote by $\mathcal{N}(h)$ the set of agents in $h$ and by $n(h)=\# \mathcal{N}(h)$ the number of those agents.

Our objective is to provide a ranking of alternative distributions of income (or any other cardinally meaningful variable) between these differentiated agents on the basis of equality. Any such income distribution, $\mathbf{x}$ say, is depicted as a collection of $k$ vectors $\left(x_{1}^{h}, \ldots, x_{n(h)}^{h}\right)$ $\in \mathbb{R}^{n(h)}$ (for $h=1, \ldots, k$ ). The criteria used in this article for comparing alternative distributions are all anonymous conditional on the agent's type. Because of this, we find convenient to index the agents in category $h$ (for $h=1, \ldots, k$ ) according to their income and to assume that $x_{i}^{h} \leq x_{i+1}^{h}$ for $i=1, \ldots, n(h)-1$. More compactly, we write a distribution $\mathbf{x}$ as $\mathbf{x}=\left\{\left(x_{1}^{h}, \ldots, x_{n(h)}^{h}\right)\right\}_{h=1}^{k}$. Since we focus on pure equality considerations in this section and the next one, we restrict our attention to income distributions $\mathbf{x}$ such that

$$
\sum_{h=1}^{k} \sum_{i=1}^{n(h)} x_{i}^{h}=I \text { for some real number } I .
$$

We let $\mathcal{D}(I)$ denote the set of all such income distributions for any given $I$. Given two distributions $\mathbf{x}, \mathbf{y} \in \mathcal{D}(I)$, we finally denote by $\underline{v}(\mathbf{x}, \mathbf{y})$ and $\bar{v}(\mathbf{x}, \mathbf{y})$ their lowest and highest income, respectively.

We find useful to illustrate the concepts and definitions proposed herein by means of the following simple examples of distributions of 6,000 dollars (say) of income among four households belonging to three different categories: couples with one child (two households), couples without children (one household), and single persons (one household). We consider specifically the three distributions $\mathbf{x}, \mathbf{y}$, and $\mathbf{z}$ described in Table 1. Applying the above notation to the distribution $\mathbf{x}$ of this table, we would have $x_{1}^{1}=1,500, x_{2}^{1}=1,600, x_{1}^{2}=1,900$, and $x_{1}^{3}=$ 1,000 .

We now introduce the main concepts between which an equivalence will be established. 
TABLE 1

THREE DISTRIBUTIONS OF INCOME BETWEEN FOUR HOUSEHOLDS BELONGING TO THREE CATEGORIES

\begin{tabular}{lccc}
\hline Distribution & Couple, One Child & Couple, No Children & Single Person \\
\hline $\mathbf{x}$ & $1,500,1,600$ & 1,900 & 1,000 \\
$\mathbf{y}$ & $1,300,1,700$ & 1,000 & 2,000 \\
$\mathbf{z}$ & $1,200,1,400$ & 1,800 & 1,600 \\
\hline
\end{tabular}

2.2. Elementary Transformations. The main elementary transformation considered is the Between-Type progressive transfer discussed in many papers, including Ebert (1997), Atkinson and Bourguignon (1982), Fleurbaey et al. (2003), Muller and Scarsini (2012), and Gravel and Moyes (2012). In order to simplify the discussion, we provide in what follows an imperfectly rigorous definition of this elementary transformation that implicitly rules out the possibility for agents not involved in the transfer to have income or categories that lie in between those of the involved agents. The Appendix provides a rigorously corre4ct-but considerably heavier-definition that allows for this possibility.

Definition 1 (Between Type Progressive Income Transfer). For any two distributions $\mathbf{x}$ and $\mathbf{y}$ in $\mathcal{D}(I)$, we say that $\mathbf{x}$ is obtained from $\mathbf{y}$ by means of a Between-Type Progressive Income Transfer (BTPIT) if there are categories $g$ and $h$ for which $g \leq h$, two agents $i^{g} \in \mathcal{N}(g)$ and $i^{h} \in \mathcal{N}(h)$ for which $y_{i^{h}}^{h}>y_{i^{g}}^{g}$ and a number $\left.\left.\alpha \in\right] 0, \frac{y_{i^{h}}^{h}-y_{i g}^{g}}{2}\right]$ such that:

(i) $x_{i^{g}}^{g}=y_{i^{g}}^{g}+\alpha$.

(ii) $x_{i^{h}}^{h}=y_{i^{h}}^{h}-\alpha$.

(iii) $x_{i}^{l}=y_{i}^{l}$ for any other pair $(i, l)$ where $l \in\{1, \ldots, k\}$ and $i \in \mathcal{N}(l)$.

A BTPIT resembles a standard one-dimensional Pigou-Dalton transfer. There is however a major difference: the beneficiary of the transfer must have both a lower income and a (weakly) lower status than the donor.

This kind of transfer is a particular case of the equalizing transformation considered by Muller and Scarsini (2012), where the transfers can be made in all dimensions. In the current setting, it would not make much sense to transfer the (ordinal) nonpecuniary variable by which agents differentiate themselves. A BTPIT is illustrated in Figure 1. Note that our definition of a BTPIT allows the donor to be of the same type as the receiver. Hence, the standard one-dimensional Pigou-Dalton transfer (conditional on type) is a particular case of BTPIT. Note also that our definition of BTPIT rules out the possibility of the amount transferred being more than half the income difference between giver and receiver.

This restriction can be eliminated by considering the following additional elementary transformation, called Favorable Income Permutation (FIP) in Gravel and Moyes (2012). ${ }^{3}$

Definition 2 (FIP). For any two distributions $\mathbf{x}$ and $\mathbf{y}$ in $\mathcal{D}(I)$, we say that $\mathbf{x}$ is obtained from $\mathbf{y}$ by means of an FIP if there are categories $g$ and $h$ for which $g<h$ and two agents $i^{g} \in \mathcal{N}(g)$ and $i^{h} \in \mathcal{N}(h)$ for which $y_{i^{h}}^{h}>y_{i^{g}}^{g}$ such that:

(i) $x_{i^{g}}^{g}=y_{i^{h}}^{h}$.

(ii) $x_{i^{h}}^{h}=y_{i^{3}}^{g}$.

(iii) $x_{i}^{l}=y_{i}^{l}$ for any other pair $(i, l)$ where $l \in\{1, \ldots, k\}$ and $i \in \mathcal{N}(l)$.

\footnotetext{
${ }^{3}$ Again, the definition provided here rules out the possibility that the transformation performed between two agents modifies the indices assigned to other agents. The proper definition that allows for this possibility is provided in the Appendix.
} 


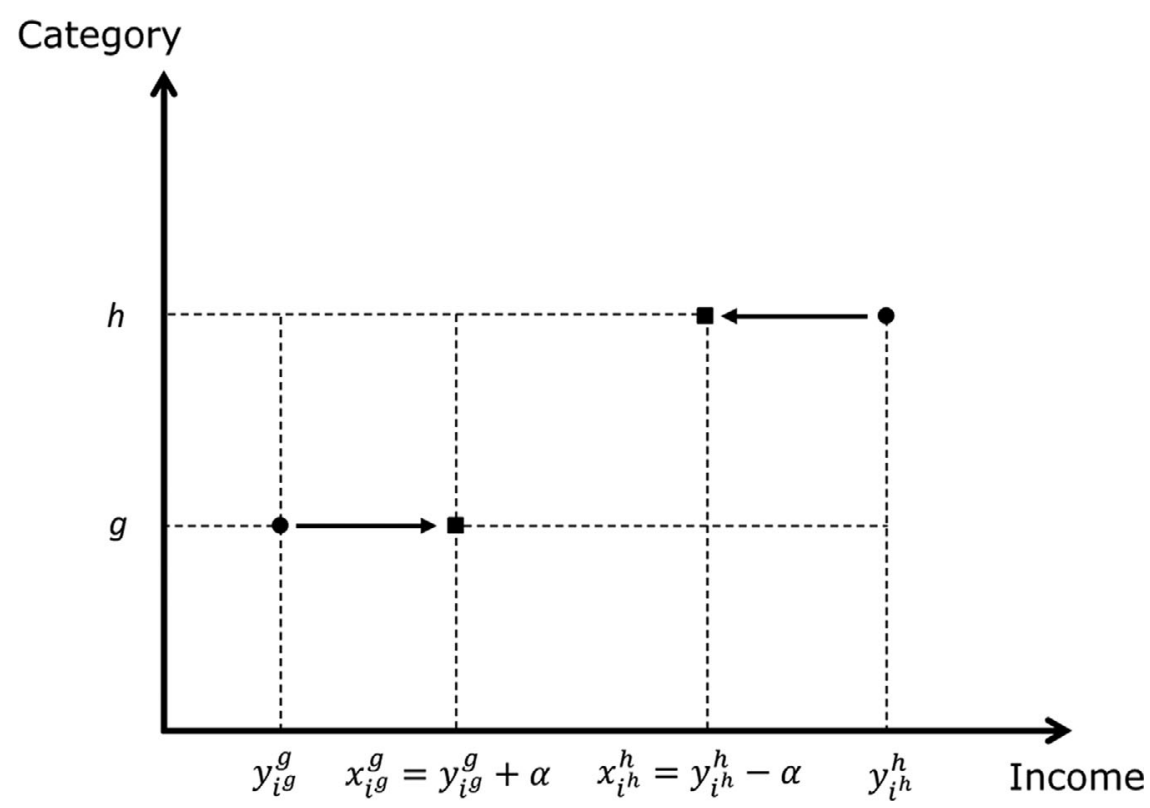

FIGURE 1

A BTPIT

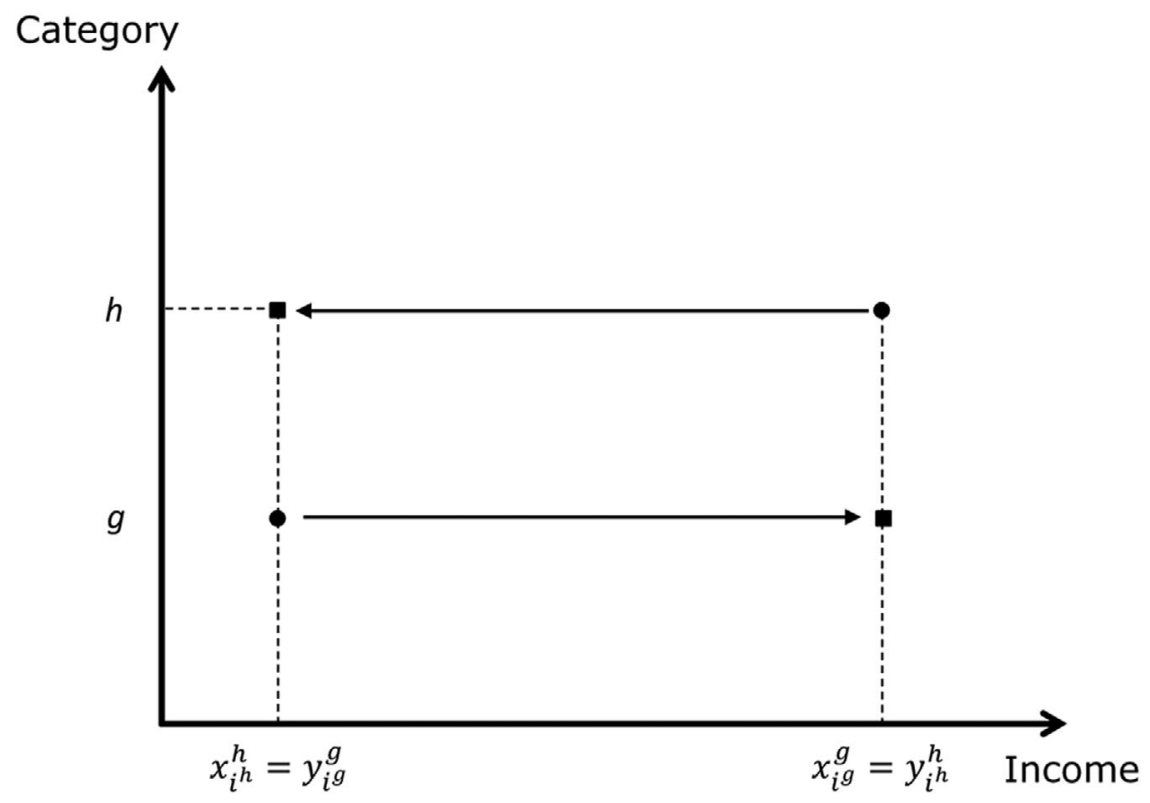

FIGURE 2

A FIP

In words, a FIP (illustrated on Figure 2) is a swap of income between a (relatively) rich agent belonging to a (relatively) high category and a poorer agent belonging to a lower category. It can thus be viewed as an extreme form of BTPIT in which the total income difference between the two agents is transferred. We emphasize however that transferring the total income difference between two agents belonging to two distinct categories is viewed 
here as a strictly equalizing transformation. This is in sharp contrast with the traditional onedimensionnal inequality measurement setting where transferring the totality of the income difference between two agents-allowed for in the definition of a Pigou-Dalton transfer by some authors, Atkinson (1987), Chakravarty and Muliere (2003), or Zheng (2007)—is only a matter of indifference.

Gravel and Moyes (2012) have shown that a BTPIT can always be decomposed into a (within-type) conventional Pigou-Dalton transfer followed by an FIP provided that a phantom agent is added. This phantom agent must be endowed with the income of the beneficiary and the category of the donor prior to the transfer.

A way to see these elementary transformations at work is to consider distributions $\mathbf{x}$ and $\mathbf{y}$ in Table 1. One can easily check that one can go from $\mathbf{y}$ to $\mathbf{x}$ by means of the following sequence of BTPITs and FIPs:

(1) Transfer 100 dollars from the single person to the poorest of the two couples with one child.

(2) Transfer 100 dollars from the richest couple with one child to the poorest such couple.

(3) Makes an FIP between the couple without children and the single person.

2.3. Utilitarian Dominance. This notion of dominance rides on the assumption that all agents of a given type transform their income into some type-dependent, ethically meaningful achievement (well-being, happiness, freedom, etc.) by means of the same (utility) function satisfying some minimal property. Specifically, the utility achieved by agent $i$ of type $h$ in distribution $\mathbf{x}$ is indicated by $U^{h}\left(x_{i}^{h}\right)$, where $U^{h}: \mathbb{R} \rightarrow \mathbb{R}$. The utilitarian rule ranks the distributions on the basis of the sum of the utilities they generate. More precisely, the utilitarian rule considers distribution $\mathbf{x}$ to be no worse than distribution $\mathbf{y}$ if and only if

$$
\sum_{h=1}^{k} \sum_{i=1}^{n(h)} U^{h}\left(x_{i}^{h}\right) \geqq \sum_{h=1}^{k} \sum_{i=1}^{n(h)} U^{h}\left(y_{i}^{h}\right) .
$$

The list of type-dependent utility functions $U^{1}, \ldots, U^{k}$ used by the utilitarian rule reflects some normative evaluation of the contribution of income to every agent's achievement, conditional on the agent's type. For the sake of robust normative evaluation, the dominance approach commonly requires a consensus among a relatively large class, $\mathcal{U}^{*}$ say, of such lists of utility functions. This gives rise to the following general notion of utilitarian dominance.

Definition 3. (Utilitarian Dominance). For any two distributions $\mathbf{x}$ and $\mathbf{y}$ in $\mathcal{D}(I)$, we say that $\mathbf{x}$ utilitarian dominates $\mathbf{y}$ for the class $\mathcal{U}^{*}$ of collections of $k$ utility functions if and only if

$$
\sum_{h=1}^{k} \sum_{i=1}^{n(h)} U^{h}\left(x_{i}^{h}\right) \geqq \sum_{h=1}^{k} \sum_{i=1}^{n(h)} U^{h}\left(y_{i}^{h}\right), \forall\left(U^{1}, \ldots, U^{k}\right) \in \mathcal{U}^{*}
$$

In this article, we specifically consider the class $\mathcal{U}^{*}$ of list of type-dependent utility functions $U^{1}, \ldots, U^{k}$ that satisfy:

$$
U^{h}(w+a)-U^{h}(w) \geq U^{h^{\prime}}\left(w^{\prime}+a\right)-U^{h^{\prime}}\left(w^{\prime}\right)
$$

for any nonnegative real number $a$, any categories $h, h^{\prime} \in\{1, \ldots, k\}$ with $h \leq h^{\prime}$, and any income pair $\left(w, w^{\prime}\right)$ such that $w \leq w^{\prime}$. In words, $\mathcal{U}^{*}$ is the class of collections of utility functions $U^{h}$ (for $h=1, \ldots, k$ ) with the property that the contribution of an additional unit of income to the individual's advantage (as measured by the function $U^{h}$ ) is decreasing with respect to both income and type. The requirement that the marginal utility of income is decreasing with 
income for any type is standard, and reflects inequality aversion. The requirement that the marginal utility of income is also decreasing with respect to the type-given income-reflects the idea that the types are ordered by their needs. With the same income, a needier agent makes a better use of an additional unit of income than a less needy agent. Observe that typedependent functions in the class $\mathcal{U}^{*}$ are neither assumed to be increasing with respect to income nor with respect to type. In particular an agent of higher type is not necessarily "betteroff" than an agent of a lower type with the same income.

In order to illustrate this notion of utilitarian dominance, consider the distributions $\mathbf{x}$ and $\mathbf{z}$ of Table 1 , and the list of three-type dependent utility functions $\left(U^{1}, U^{2}, U^{3}\right)$ defined by $U^{2}(x)=U^{3}(x)=1,500, U^{1}(x)=\min \{x, 1,500\}$. It is clear that this list belongs to $\mathcal{U}^{*}$. Observe that one has:

$$
\begin{array}{r}
\sum_{h=1}^{3} \sum_{i=1}^{n(h)} U^{h}\left(x_{i}^{h}\right)=1500+1500+1500+1500=6000 \\
\text { and } \sum_{h=1}^{3} \sum_{i=1}^{n(h)} U^{h}\left(z_{i}^{h}\right)=1200+1400+1500+1500=5600
\end{array}
$$

so that $\sum_{h=1}^{3} \sum_{i=1}^{n(h)} U^{h}\left(x_{i}^{h}\right)>\sum_{h=1}^{3} \sum_{i=1}^{n(h)} U^{h}\left(z_{i}^{h}\right)$. However, if one considers the collection $\left(V^{1}, V^{2}, V^{3}\right)$ of utility functions defined by $V^{1}(x)=V^{2}(x)=V^{3}(x)=\min \{x, 1200\}$ (that also trivially belong to $\left.\mathcal{U}^{*}\right)$, then one has the reverse inequality:

$$
\sum_{h=1}^{3} \sum_{i=1}^{n(h)} V^{h}\left(x_{i}^{h}\right)=4600<\sum_{h=1}^{3} \sum_{i=1}^{n(h)} V^{h}\left(z_{i}^{h}\right)=4800 .
$$

Hence, $\mathbf{x}$ and $\mathbf{z}$ are not comparable for the utilitarian dominance criterion applied to the class of lists of utility functions satisfying (3).

2.4. OPG Dominance. The OPG criterion has been proposed by Bourguignon (1989) for comparing income distributions between households of differing sizes. In order to discuss this criterion in the current context, we first define the set $\mathcal{V} \subset \mathbb{R}^{k}$ by:

$$
\mathcal{V}=\left\{\left(v_{1}, \ldots, v_{k}\right) \in \mathbb{R}^{k}: v_{1} \geq v_{2} \geq \ldots \geq v_{k}\right\}
$$

Set $\mathcal{V}$ comprises all combinations of poverty lines (one such line for every type) that are weakly decreasing with respect to type. Given this set, we define the OPG dominance criterion as follows.

Definition 4. (OPG Dominance). For any two distributions $\mathbf{x}$ and $\mathbf{y}$ in $\mathcal{D}(I)$, we say that $\mathbf{x}$ dominates $\mathbf{y}$ for the OPG criterion, denoted by $\mathbf{x} \succsim^{O P G} \mathbf{y}$, if the following holds:

$$
P^{\mathbf{x}}\left(v_{1}, \ldots, v_{k}\right) \leq P^{\mathbf{y}}\left(v_{1}, \ldots, v_{k}\right), \forall\left(v_{1}, \ldots, v_{k}\right) \in \mathcal{V},
$$

where $P^{\mathbf{x}}\left(v_{1}, \ldots, v_{k}\right):=\sum_{h=1}^{k} \sum_{i=1}^{n(h)} \max \left(v_{h}-x_{i}^{h}, 0\right)$.

In words, $\mathbf{x}$ dominates $\mathbf{y}$ for the OPG criterion if, for all possible poverty lines that are nonincreasing with respect to agent's type, the minimum income required to eliminate poverty defined by these lines is lower in $\mathbf{x}$ than in $\mathbf{y}$. The requirement for the poverty lines to be nonincreasing with the type is quite intuitive. It simply says that with a given income, an agent is (weakly) more likely to be considered poor when his/her type is low than when it is high. In 
order to illustrate OPG dominance, consider distributions $\mathbf{y}$ and $\mathbf{z}$ of Table 1 . Choosing first the three nonincreasing poverty lines $\left(v_{1}, v_{2}, v_{3}\right)=(1300,1000,1000)$, we observe that:

$$
P^{\mathbf{y}}\left(v_{1}, v_{2}, v_{3}\right)=0
$$

whereas:

$$
P^{\mathbf{z}}\left(v_{1}, v_{2}, v_{3}\right)=100
$$

Hence, $\mathbf{z}$ does not dominate $\mathbf{y}$ as per the OPG criterion. However, if one chooses instead the three nonincreasing poverty lines $\left(v_{1}^{\prime}, v_{2}^{\prime}, v_{3}^{\prime}\right)=(1200,1200,1200)$, one has:

$$
P^{\mathbf{y}}\left(v_{1}^{\prime}, v_{2}^{\prime}, v_{3}^{\prime}\right)=200
$$

whereas:

$$
P^{\mathrm{z}}\left(v_{1}^{\prime}, v_{2}^{\prime}, v_{3}^{\prime}\right)=0
$$

so that $\mathbf{y}$ does not dominate $\mathbf{z}$ either. Hence $\mathbf{y}$ and $\mathbf{z}$ are not comparable by OPG dominance.

Although OPG dominance requires a comparison of the poverty gap between two distributions for all lists of ordered poverty lines in $\mathcal{V}$ (a noncountable set), it is nonetheless easily implementable; see, for example, Decoster and Ooghe (2006) or Gravel et al. (2009). One way of implementation is to restrict attention, as just done for the distributions $\mathbf{y}$ and $\mathbf{z}$ of Table 1 , to the (finite) subset of lists of poverty lines in $\mathcal{V}$ that are actually observed in the two distributions under comparison. Another is to use the ingenious alternative formulation of the OPG dominance criterion proposed by (Bourguignon, 1989, p. 74, equation (12)) via an iterative procedure based on the largest difference in poverty gap between two distributions for all poverty lines above any arbitrary threshold. In the next subsection, we introduce an alternative dominance criterion that is somewhat analogous to Lorenz dominance and that is also easily implementable.

2.5. Cumulative Lowest Incomes (CLI) Dominance. In the classical case of income distributions among homogeneous agents, it is well known that poverty gap dominance is equivalent to the requirement, known as Lorenz dominance, that the sum of income of the $m$ poorest agents is larger in the dominating than in the dominated distribution no matter what $m$ is (see Berge, 1959, for instance). In this subsection, we introduce a similar dominance criterion based on the sum of incomes of the $m$ poorest agents when those agents are differentiated by a non-income characteristic. This is challenging because there is no obvious way to define who the $m$ poorest agents are in such a case. For example, is a couple without children earning a weekly income of $\$ 350$ really poorer than a couple with one child earning $\$ 500$ ?

Any definition of the $m$ poorest agents rides on a ranking of the agents in terms of poverty. Once a poverty ranking of the agents is defined, a set of the $m$ poorest agents is simply a collection of $m$ agents such that if an agent is in the collection and if another agent is poorer than this agent as per the ranking, then this other agent must also be in the collection. When agents are homogenous in all other dimensions than income, the poverty ranking of the agents is clear and complete: Agent $A$ is poorer than Agent $B$ if and only if $A$ has a lower income than $B$. Under this poverty ranking, there can be only one set of $m$ poorest agents: it is the set of agents who have the $m$-lowest incomes.

In the current setting where agents are vertically differentiated with respect to a nonincome characteristic, we propose to consider agent $A$ to be (weakly) poorer than agent $B$ if and only if $A$ both has a (weakly) lower income and belongs to a (weakly) lower category than $B$. This poverty ranking is clearly incomplete. As a result, it will typically generate several sets of the $m$-poorest agents satisfying the requirement that whenever they contain an 
agent, they also contain all agents who are poorer than this agent as per the ranking. Any such set of $m$-poorest agents can therefore be represented as a $k$-tuple of ranks $\left(r_{1}, \ldots, r_{k}\right)$, below which agents of the different categories are considered to be poor. This leads us to define, for any given $m \leq n$, the family $\Pi(\mathbf{x}, m)$ of admissible sets of $m$-poorest agents in distribution $\mathbf{x} \in$ $\mathcal{D}(I)$ as follows:

$$
\Pi(\mathbf{x}, m)=\left\{\left(r_{1}, \ldots, r_{k}\right): r_{h} \in\{0, \ldots, n(h)\}, \sum_{h=1}^{k} r_{h}=m, x_{r_{h}+1}^{h}>x_{r_{h^{\prime}}}^{h^{\prime}} \forall h^{\prime}>h\right\}
$$

with the convention that $x_{0}^{h}=\underline{v}(\mathbf{x}, \mathbf{y})$ and $x_{n_{h}+1}^{h}=\bar{v}(\mathbf{x}, \mathbf{y})$ for every $h$. In words, $\Pi(\mathbf{x}, m)$ is the family of all combinations $\left(r_{1}, \ldots, r_{k}\right)$ of type-dependent poverty ranks of agents that have two characteristics. First, the ranks must sum to $m$ so as to identify $m$ "poorest" agents in the whole distribution. Second, the ranks must be such that if they define an agent as poor, they must also consider as poor any agent in a lower category who has a lower income.

Observe that the set of $m$-poorest agents in the usual sense-that is the agents who earn the $m$ lowest incomes irrespective of their type-will always be $a$ set of $m$-poorest agents as per the definition of the family $\Pi(\mathbf{x}, m)$. Because of this, the family $\Pi(\mathbf{x}, m)$ is never empty. However, and contrary to the usual definition of the set of the $m$-poorest agents, there will typically be many lists of type-dependent ranks in $\Pi(\mathbf{x}, m)$. Moreover, these lists may vary across distributions. In order to illustrate this, consider distributions $\mathbf{x}$ and $\mathbf{z}$ in Table 1. There are two ways of defining the poorest household as per П(.) in $\mathbf{x}$. One is of course to consider the absolutely poorest household-who is the single person in $\mathbf{x}$-as the poorest. This would correspond to the list of ranks $(0,0,1)$. The only other possibility compatible with the second inequality of Expression (6) is to consider that the poorest of the two couples with one child is the poorest household. This would correspond to the list of ranks $(1,0,0)$. The possibility of considering the couple without children as the poorest, which would correspond to the list of ranks $(0,1,0)$, is ruled out by the definition of $\Pi($.$) because both couples with one child (who$ would be above their assigned rank and therefore not poor under this specification) have a lower income than the couple without children. We thus have $\Pi(\mathbf{x}, 1)=\{(1,0,0),(0,0,1)\}$. However there is only one way to define the poorest household in distribution $\mathbf{z}$. It is the household with the lowest income, here the poorest of the two couples with one child. Any other specification of the ranks that sum to one would indeed violate the second inequality of Expression (6). Hence, we have $\Pi(\mathbf{z}, 1)=\{(1,0,0)\}$.

Using this definition of the set $\Pi(., m)$ of $m$ poorest agents, we propose the following notion of CLI dominance.

Definition 5. For any two distributions $\mathbf{x}$ and $\mathbf{y}$ in $\mathcal{D}(I)$, we say that $\mathbf{x}$ dominates $\mathbf{y}$ for the CLI criterion, which we denote as $\mathbf{x} \succsim^{C L I} \mathbf{y}$ if, for any $m \in\{1, \ldots, n\}$, and any $\left(r_{1}, \ldots, r_{k}\right) \in$ $\Pi(\mathbf{x}, m)$, there exists $\left(r_{1}^{\prime}, \ldots, r_{k}^{\prime}\right) \in \Pi(\mathbf{y}, m)$ such that

$$
\sum_{h=1}^{k} \sum_{i \leq r_{h}} x_{i}^{h} \geq \sum_{h=1}^{k} \sum_{i \leq r_{h}^{\prime}} y_{i}^{h}
$$

and

$$
\sum_{g=1}^{h} r_{g}^{\prime} \geq \sum_{g=1}^{h} r_{g}
$$

for any $h=1, \ldots, k$. 
In words, $\mathbf{x}$ dominates $\mathbf{y}$ for the CLI criterion if, for any $m$, and any admissible set of $m$ poorest agents in $\mathbf{x}$, there is an admissible set of $m$ poorest agents in $\mathbf{y}$ who have a lower sum of income than the $m$ poorest agents in $\mathbf{x}$ (Condition (7)) and who are such that, for every category $h$, the sum, over all categories below $h$, of the category-specific ranks below which agents are considered poor is larger in $\mathbf{y}$ than in $\mathbf{x}$ (Condition (8)). In essence, this latter condition requires the definition of the poorest agents to be such that the number of agents considered poor in the bottom $h$ categories is larger in the dominated than in the dominating distribution for all $h$.

In order to illustrate this latter condition, which does not appear in the definition of standard Lorenz dominance, consider again distributions $\mathbf{x}$ and $\mathbf{z}$ in Table 1 . It is easy to see that these two distributions cannot be compared by the CLI criterion. Indeed, we first observe that $\mathbf{x}$ does not dominate $\mathbf{z}$. Such a dominance would make it possible to find, for any $m$ and any admissible definition of the $m$ poorest agents in $\mathbf{x}$, an admissible collection of the $m$ poorest agents in $\mathbf{z}$ with respect to which the two inequalities (8) and (7) hold. But this is not possible, even for $m=1$. If we take the single person as the poorest agent in $\mathbf{x}$ (corresponding to the list of ranks $(0,0,1)$ in $\Pi(\mathbf{x}, 1))$, we observe that this single person has a lower income than that of the poorest of the two couples with one child in $\mathbf{z}$, who is as we know the only admissible poorest agent in this distribution. Hence, Inequality (7) would be violated in that case. To see that $\mathbf{z}$ does not dominate $\mathbf{x}$ either, we again only consider the case $m=1$ and the only admissible definition of the poorest agent in $\mathbf{z}$ (the poorest of the two couples with one child). Only one of two admissible poorest agents in $\mathbf{x}$-the single person-has a lower income than the poorest of the two couples with one child in $\mathbf{z}$. However the list of ranks that would make the single person the poorest household in $\mathbf{x}$-that is, $(0,0,1)$-violates Inequality (8) as compared to $(1,0,0)$. In fact, defining the single person as the poorest household in $\mathbf{x}$ and the poorest of the two couples with one child as the poorest household in $\mathbf{z}$, amounts to assuming that there are more poor agents in in the bottom category (couples with one child) in $\mathbf{z}$ than in $\mathbf{x}$, in violation to (8).

The CLI dominance criterion is no doubt more difficult to implement empirically than its unidimensional Lorenz dominance cousin. For one thing, the criterion cannot be checked by simply comparing two curves. Yet the CLI dominance criterion is easy to implement through the following procedure, suggested by the fact that having Lorenz dominance between two distributions is a necessary condition for CLI dominance. Check first for standard Lorenz dominance. If there is no such dominance, then there is no CLI dominance and the procedure stops. If, however, there is Lorenz dominance, then the Lorenz dominating distribution could be a CLI dominant distribution. Start therefore with the Lorenz dominating distribution and, for any $m=1, \ldots, n$, identify all admissible (under Condition (6)) combinations of ranks that sum to $m$ in that distribution. For any such combination, find all admissible (under Condition (6)) combinations of ranks that sum to $m$ in the Lorenz dominated distribution and that satisfy inequality (8). If there are no such combinations, then there is no dominance and the procedure stops. If there are such combinations, then select from them the combination of ranks associated with the smallest sum of incomes, and compare this smallest sum of incomes with that associated with the initial combination of ranks in the potentially dominating distribution. If the comparison of the two sums violates Condition (7), then there is no dominance and the procedure stops. Otherwise, the procedure continues for all $m$, in which case we conclude that there is CLI dominance.

\section{MAIN RESULT}

The main theorem proved in this article is the following. 
(i) It is possible to go from $\mathbf{y}$ to $\mathbf{x}$ by a finite sequence of BTPIT and/or FIP.

(ii) $\mathbf{x}$ utilitarian dominates $\mathbf{y}$ for the class $\mathcal{U}^{*}$.

(iii) $\mathbf{x} \succsim^{O P G} \mathbf{y}$.

(iv) $\mathbf{x} \succsim^{C L I} \mathbf{y}$.

The proof of this theorem proceeds in several steps. The first, establishing that $(i)$ implies (ii) and that (ii) implies (iii), is easy and quite well-known; see, for example, Ebert (1997) or Gravel and Moyes (2012). It is formally stated as Proposition A.1 in Subsection A.2 in the Appendix, where it is also proved for the sake of completeness. In Subsection A.3 in the Appendix we also state and prove the equivalence between (iii) and (iv).

The most difficult part of the proof is, however, the implication that statement (iii) implies statement $(i)$. This proof is lengthy and has been for the most part developed in the Appendix. We find nonetheless useful to provide in this section an overall picture of the proof strategy. Proving that statement (iii) implies statement $(i)$ amounts in effect to constructing an algorithm for going from a distribution $\mathbf{y}$ to a distribution $\mathbf{x}$ by a finite sequence of either BTPIT and/or FIP that is based solely on the information that $\mathbf{x} \succsim^{O P G} \mathbf{y}$. The difficulty of the construction rides on the fact that, in every step of the algorithm, either a BTPIT or an FIP must be performed in such a way that the result of the elementary transformation remains dominated by distribution $\mathbf{x}$. In so doing, it is important to identify, at each step of the algorithm, which of the two transformations can be made. The main contribution of our proof strategy is to propose a diagnostic tool, introduced in Subsection 3.1, that can be used for that purpose. In a nutshell, our diagnostic tool checks whether there is some margin to make a transfer whose beneficiary is the poorest individual in the lowest category. If the margin is there, then a further refinement of the diagnostic tool enables us to choose to perform either a transfer, or a permutation (see Propositions 1 and 2). If the margin is not there, then a permutation is absolutely needed (see Proposition 3). Finally, in Subsection 3.2, we sketch how our algorithm works and prove its finiteness.

3.1. Identifying Which Elementary Operation Is Possible: A Diagnostic Tool. Our diagnostic tool is based on the critical value $v_{1}^{c}$ defined as follows:

$$
v_{1}^{c}:=\inf \left\{v_{1}>y_{1}^{1}: \exists v_{2}, \ldots, v_{k} \text { s.t. } \mathbf{v}=\left(v_{1}, \ldots, v_{k}\right) \in \mathcal{V} \text { and } P^{\mathbf{x}}(\mathbf{v})=P^{\mathbf{y}}(\mathbf{v})\right\} .
$$

In words, $v_{1}^{c}$ is the lowest poverty threshold above the smallest income in the lowest category in the dominated distribution $\mathbf{y}$ that can be part of a collection of ordered poverty thresholds for which the OPG in distributions $\mathbf{x}$ and $\mathbf{y}$ is the same. The quantity $v_{1}^{c}$ is well-defined because the set:

$$
\left\{v_{1}>y_{1}^{1}: \exists v_{2}, \ldots, v_{k} \text { s.t. } \mathbf{v}=\left(v_{1}, \ldots, v_{k}\right) \in \mathcal{V} \text { and } P^{\mathbf{x}}(\mathbf{v})=P^{\mathbf{y}}(\mathbf{v})\right\}
$$

is not empty (it contains $\bar{v}(\mathbf{x}, \mathbf{y})$ ) and is also bounded from below (by $\underline{v}(\mathbf{x}, \mathbf{y})$ ). Two mutually exclusive cases are possible:

(A) $v_{1}^{c}>y_{1}^{1}$

(B) $v_{1}^{c}=y_{1}^{1}$.

As will be shown, if case $(A)$ holds, there is some margin to make a strict BTPIT to the poorest agent in category 1 (endowed with $y_{1}^{1}$ ) in such a way that the after-transfer distribution remains dominated by $\mathbf{x}$ as per the OPG criterion. This however does not rule out the possibility that an FIP involving an agent in category 1 can also be performed while preserving the OPG dominance by $\mathbf{x}$. If both an FIP and a BTPIT are possible, then our algorithmic procedure will always choose to perform the FIP. ${ }^{4}$ If on the other hand case $(B)$ holds, then we show the

\footnotetext{
${ }^{4}$ We explain in Subsection 3.2 why we make this choice.
} 
possibility of involving the poorest agent of the lowest category in a FIP while preserving the OPG dominance of $\mathbf{x}$ over the newly obtained distribution. Starting from $v_{1}^{c}$, one can then recursively define the quantities $v_{h}^{c}$, for $h=2, \ldots, k$ as

(10) $v_{h}^{c}=\min \left\{v_{h} \geq \underline{v}(\mathbf{x}, \mathbf{y}): \exists v_{h+1}, \ldots, v_{k}\right.$ s.t. $\left.\mathbf{v}=\left(v_{1}^{c}, . ., v_{h-1}^{c}, v_{h}, . ., v_{k}\right) \in \mathcal{V}, P^{\mathbf{x}}(\mathbf{v})=P^{\mathbf{y}}(\mathbf{v})\right\}$.

We refer to vector $\mathbf{v}^{c}:=\left(v_{1}^{c}, \ldots, v_{k}^{c}\right) \in \mathcal{V}$ as the critical vector. This vector can be thought of as the lowest "relevant" ordered list of poverty lines that yield the same poverty in the two distributions. The critical value $v_{1}^{c}$, as well as the associated critical vector $\mathbf{v}^{c}$, are mathematical diagnostic tools enabling us to identify the elementary transformation that can be performed at every step of the algorithm. Given two distributions, it is easy to find this vector. Consider for instance the distributions $\mathbf{x}$ and $\mathbf{y}$ in Table 1 . Here $y_{1}^{1}=1300$. We have:

$$
P^{\mathbf{y}}(\mathbf{v})-P^{\mathbf{x}}(\mathbf{v})=\left\{\begin{array}{cll}
v_{2}-v_{3}+v_{1}-1300>0 & \text { if } & \left.\left.v_{1} \in\right] 1300,1500\right] \\
v_{2}-v_{3}+200 \geq 200 & \text { if } & v_{1} \in[1500,1600] \\
v_{2}+v_{3}+1800-v_{1} \geq 100 & \text { if } & v_{1} \in[1600,1700] \\
v_{2}-v_{3}+100 \geq 100 & \text { if } & v_{1} \in[1700,1900] \\
v_{2}-v_{3}+100-\left(v_{2}-1900\right)_{+} & \text {if } & v_{1} \in[1900,2000]
\end{array}\right.
$$

Hence, in the relevant set $\left\{\left(v_{1}, v_{2}, v_{3}\right) \in \mathcal{V}: v_{1}>y_{1}^{1}, v_{1} \leq \bar{v}(\mathbf{x}, \mathbf{y})\right\}$ the poverty difference is equal to zero if and only if $v_{1}=v_{2}=v_{3}=2000.2000$ is therefore the only value of $v_{1}$ which is both strictly larger than $y_{1}^{1}$ and part of a poverty line vector $\mathbf{v}=\left(v_{1}, v_{2}, v_{3}\right)$ for which the poverty gap is the same in the two distributions. Consequently $v_{1}^{c}=2000$, and $\mathbf{v}^{c}=$ $(2000,2000,2000)$. An example where the critical value $v_{1}^{c}$ is equal to $y_{1}^{1}$ is provided later on, see Figure 7.

3.1.1. Case $(A): v_{1}^{c}>y_{1}^{1}$. We establish in this case the following proposition which says that, if $\mathbf{x} \succsim^{O P G} \mathbf{y}$ holds, then it is possible to find a distribution $\widehat{\mathbf{x}}$ that is OPG dominated by $\mathbf{x}$ and that is obtained from $\mathbf{y}$ by a BTPIT.

Proposition 1. Let $\mathbf{x}$ and $\mathbf{y}$ be two distributions in $\mathcal{D}(I)$ such that $\mathbf{x} \succsim^{O P G} \mathbf{y}$. Suppose that $v_{1}^{c}>y_{1}^{1}$. Then, there exists a distribution $\widehat{\mathbf{x}} \in \mathcal{D}(I)$ such that:

- $\mathbf{x} \succsim O P G \widehat{\mathbf{x}}$,

- $\widehat{\mathbf{x}}$ is obtained from $\mathbf{y}$ by a BTPIT involving some agent $i^{j} \in \mathcal{N}(j)$ for some category $j \in$ $\{1, \ldots, k\}$ and agent $1 \in \mathcal{N}(1)$.

This proposition (proved in the Appendix, Subsection A.5) identifies a particular category $j \geq 1$ and a particular agent in that category (labeled $i^{j}$ ) that can transfer a strictly positive quantity of income to the poorest agent in category 1 . Since we establish this proposition with the objective of constructing a finite sequence of such transfers, it is important for the sequence not to be unduly long and, therefore, for each transfer in the sequence to be as large as possible. This leads to the following notion of a maximal transfer that is exploited crucially in the argument.

Definition 6. Let $\mathbf{x}$ and $\mathbf{y}$ be two distributions in $\mathcal{D}(I)$ such that $\mathbf{x} \succsim^{O P G} \mathbf{y}$. Suppose that $\hat{\mathbf{x}}$ is obtained from $\mathbf{y}$ by means of a BTPIT where agent $i^{h} \in \mathcal{N}(h)$ transfers $\alpha_{0} \geq 0$ to agent $i^{g} \in$ $\mathcal{N}(g)(g \leq h)$ and that $\hat{\mathbf{x}} \precsim O P G \mathbf{x}$. We say that this transfer is maximal (with respect to $\mathbf{x}$ ) if any of the following conditions holds:

(MT1) Equalizing transfer: there exist $i, i^{\prime} \in \mathcal{N}(h)$ such that $\widehat{x}_{i^{\prime}}^{h}=x_{i}^{h}$ or there exists some $i, i^{\prime} \in \mathcal{N}(g)$ such that $\hat{x}_{i^{\prime}}^{g}=x_{i}^{g}$ (i.e., one of the two agents involved in the transfer obtains the income that they will have in final distribution $\mathbf{x}$ ). 


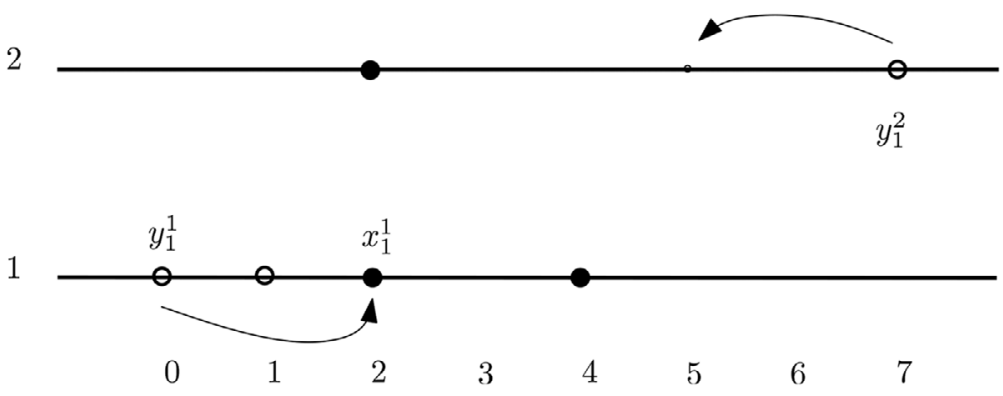

FIGURE 3

EQUALIZING TRANSFER

(MT2) Breaking transfer: for any $\alpha<\alpha_{0}$, the transfer of amount $\alpha$ from agent $i^{h} \in \mathcal{N}(h)$ to agent $i^{g} \in \mathcal{N}(g)$ is not equalizing. Additionally for any $\alpha>\alpha_{0}$ the distribution obtained by making a transfer of amount $\alpha$ from agent $i_{h}$ to agent $i_{g}$ is not dominated by $\mathbf{x}$ as per the OPG dominance criterion.

(MT3) Half transfer: $\alpha_{0}=\left(y_{i^{h}}^{h}-y_{i^{g}}^{g}\right) / 2$ and, for any $\alpha<\alpha_{0}$, the transfer of amount $\alpha$ from agents $i^{h} \in \mathcal{N}(h)$ to agent $i^{g} \in \mathcal{N}(g)$ is not equalizing.

Observe that a maximal transfer in the sense of this definition is uniquely defined. We now illustrate these three possible types of maximal transfers in a simple setting where $k=2$.

Example 1 (Equalizing Transfer). Consider the distributions where $\mathcal{N}(1)=\{1,2\}$ and $\mathcal{N}(2)=\{1\}$ and where:

$$
\begin{gathered}
y_{1}^{1}=0, y_{2}^{1}=1, y_{1}^{2}=7, \\
x_{1}^{1}=2, x_{2}^{1}=4, \text { and } x_{1}^{2}=2 .
\end{gathered}
$$

It can be checked that $\mathbf{x} \succsim^{O P G} \mathbf{y}$ and that $v_{1}^{c}=7>y_{1}^{1}$.

One can see that it is possible for the unique agent in $\mathcal{N}(2)$ to transfer 2 units of income to agent 1 without breaking any OPG inequalities. Such a transfer, depicted on Figure 3, is equalizing. Observe that it could have been possible to transfer even more than 2 without breaking the OPG inequality. Yet doing so in the algorithm would be unnecessary because after receiving 2 units of income, agent 1 of category 1 has the income of the target distribution $\mathbf{x}$.

Example 2 (Breaking Transfer). Consider the distributions where $\mathcal{N}(1)=\{1,2\}=\mathcal{N}(2)$ and where:

$$
\begin{gathered}
y_{1}^{1}=0=y_{1}^{2}, y_{2}^{1}=7=y_{2}^{2}, \\
x_{1}^{1}=5, x_{2}^{1}=6, x_{1}^{2}=1 \text { and } x_{2}^{2}=2 .
\end{gathered}
$$

We have $\mathbf{x} \succsim^{O P G} \mathbf{y}$ and, again, it turns out that $v_{1}^{c}=7>y_{1}^{1}$. It is possible for agent 2 in category 2 to transfer 3 units of income to agent 1 in category 1 . Making this transfer changes the distribution from $\mathbf{y}$ to $\widehat{\mathbf{x}}$, where $\widehat{\mathbf{x}}$ is defined by:

$$
\widehat{x}_{1}^{1}=3, \widehat{x}_{2}^{1}=7, \widehat{x}_{1}^{2}=0 \text {, and } \widehat{x}_{2}^{2}=4 \text {. }
$$




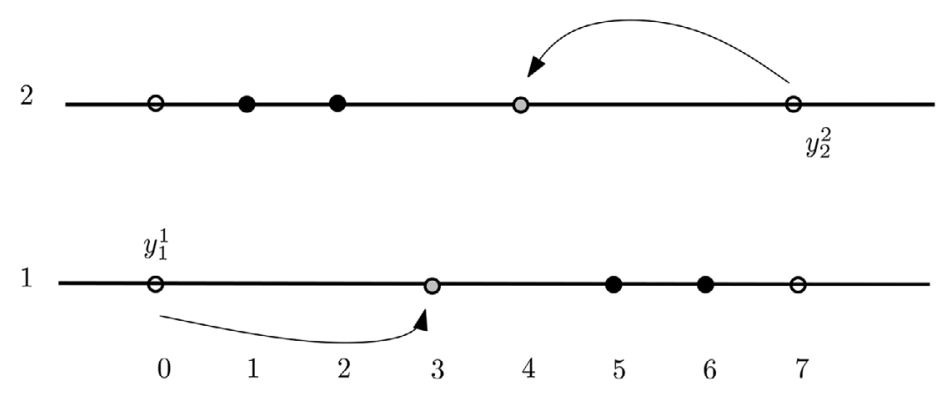

FIGURE 4

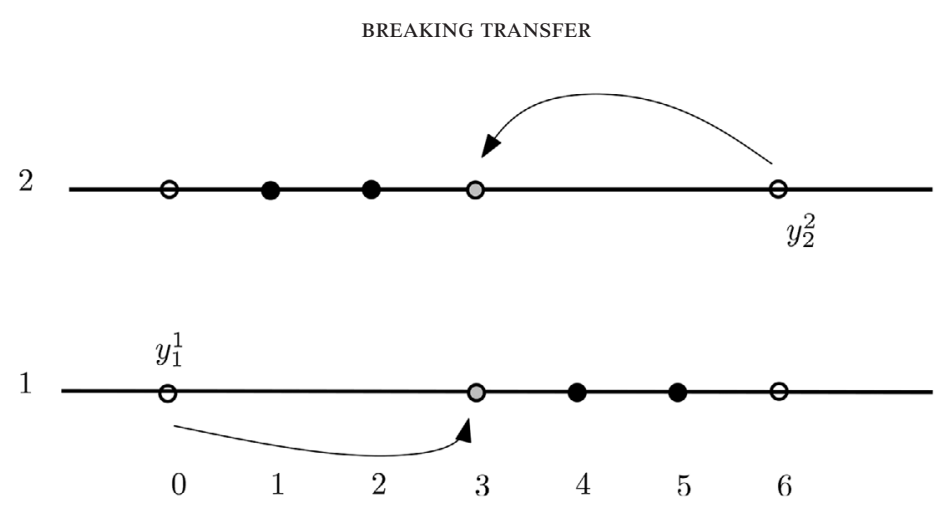

Figure 5

HALF TRANSFER

As can be seen, $\mathbf{x} \succsim^{O P G} \widehat{\mathbf{x}}$. Yet, as shown in the proof provided in the Appendix, transferring $3+\varepsilon$ (for any $\varepsilon \in] 0,1 / 2]$ ) would destroy this OPG dominance relation. The breaking transfer of this example is depicted in Figure 4.

Example 3 (Half Transfer). (see Figure 5 for an graphical depiction). Consider the distributions where $\mathcal{N}(1)=\{1,2\}=\mathcal{N}(2)$ and:

$$
\begin{gathered}
y_{1}^{1}=0=y_{1}^{2}, y_{2}^{1}=6=y_{2}^{2}, \\
x_{1}^{1}=4, x_{2}^{1}=5, x_{1}^{2}=1, \text { and } x_{2}^{2}=2 .
\end{gathered}
$$

We have $\mathbf{x} \succsim^{O P G} \mathbf{y}$ and $v_{1}^{c}=6>x_{1}^{1}$. It is possible for agent 2 in category 2 to transfer 3 to the poorest agent in category 1 -which is precisely half of their income difference-without breaking any of the inequalities that define OPG dominance.

Proposition 1 shows that a BTPIT benefiting the poorest agent in category 1 in the dominated distribution $\mathbf{y}$ can be performed in such a way that the distribution obtained after the transfer remains dominated by $\mathbf{x}$ as per the OPG criterion. However this proposition does not rule out the alternative possibility of performing an FIP between two individuals in such a way as to preserve the OPG dominance of the distribution obtained after performing this operation by $\mathbf{x}$. In the next proposition, we identify a circumstance which also entails the possibility of performing an FIP. 


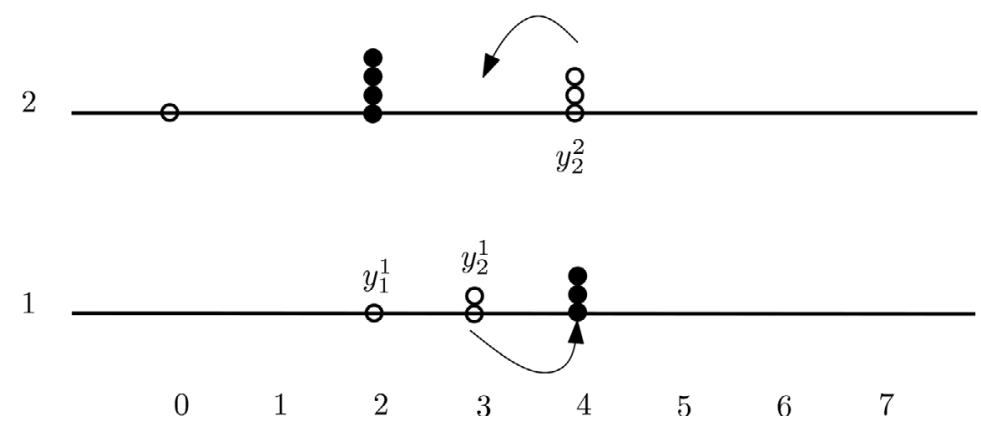

FIGURE 6

AGENT 2 IN CATEGORY 2 CAN EXCHANGE INCOME WITH AGENT 2 IN CATEGORY 1

Proposition 2. Let $\mathbf{x}$ and $\mathbf{y}$ be two distributions in $\mathcal{D}(I)$ such that $\mathbf{x} \succsim^{O P G} \mathbf{y}$. Suppose that $v_{1}^{c}>y_{1}^{1}$. Let $h_{0} \in\{1, \ldots, k\}$ be a category such that $v_{h_{0}+1}^{c}<v_{h_{0}}^{c}=v_{1}^{c}$ (with the convention that $\left.v_{k+1}^{c}=\underline{v}(\mathbf{x}, \mathbf{y})\right)$. Suppose also that

- $\forall i \in \mathcal{N}(1), x_{i}^{1}>v_{1}^{c}$

- For any category $h$ such that $h_{0} \geq h \geq 2$, we have $y_{i}^{h} \neq v_{1}^{c}$ for every $i$; $\widehat{\mathbf{x}}$.

Then there exists a distribution $\widehat{\mathbf{x}} \in \mathcal{D}(I)$ such that $\widehat{\mathbf{x}}$ is obtained from $\mathbf{y}$ by an FIP and $\mathbf{x} \succsim^{O P G}$

This proposition, proved in Subsection A.5 of the Appendix, rides heavily on the technical lemma A.3, stated and proved in the very same section of the Appendix, that provides general conditions on distributions $\mathbf{x}$ and $\mathbf{y}$ under which an FIP can be done without breaking the OPG dominance. A careful look at the proof of the proposition shows that the beneficiary of the FIP in Proposition 2 is not the poorest individual in category 1. It is another agent in category 1 whose income is equal to $v_{1}^{c}$.

In order to illustrate the possibility, identified by Proposition 2, where both a BTPIT and an FIP are possible when $v_{1}^{c}>y_{1}^{1}$, consider the case where $k=2, \mathcal{N}(1)=\{1,2,3\}, \mathcal{N}(2)=$ $\{1,2,3,4\}$ and $\mathbf{y}$ and $\mathbf{x}$ are defined by:

$$
y_{1}^{1}=2, y_{2}^{1}=y_{3}^{1}=3, y_{1}^{2}=0, y_{2}^{2}=y_{3}^{2}=y_{4}^{2}=4
$$

and:

$$
x_{1}^{1}=x_{2}^{1}=x_{3}^{1}=4, x_{1}^{2}=x_{2}^{2}=x_{3}^{2}=x_{4}^{2}=2 .
$$

As can be seen, $\mathbf{x} \succsim^{O P G} \mathbf{y}, v_{1}^{c}=v_{2}^{c}=3$, and $y_{1}^{1}=2$. According to Proposition 1 , agent 2 in category 2 can transfer some income to agent 1 in category 1 . However, Proposition 2 states that an FIP is also possible. Indeed the conditions of this proposition are satisfied since $x_{i}^{1}=4>v_{1}^{c}$ for all $i \in \mathcal{N}(1)$. We also have that $y_{i}^{2} \neq 3$ for all $i \in \mathcal{N}(2)$. By virtue of Proposition 2, the distribution obtained after exchanging income 4 of agent 2 in category 2 with income 3 of agent 2 in category 1 remains dominated by distribution $\mathbf{x}$. The situation is illustrated in Figure 6.

3.1.2. Case $(B): v_{1}^{c}=y_{1}^{1}$. Using crucially Lemmas 3 and 7 of the Appendix, we now establish, in the following proposition also proved in Subsection A.6 of the Appendix, the possibility of performing an FIP on the initial distribution $\mathbf{y}$ while maintaining OPG dominance of the newly created distribution by $\mathbf{x}$. 
2
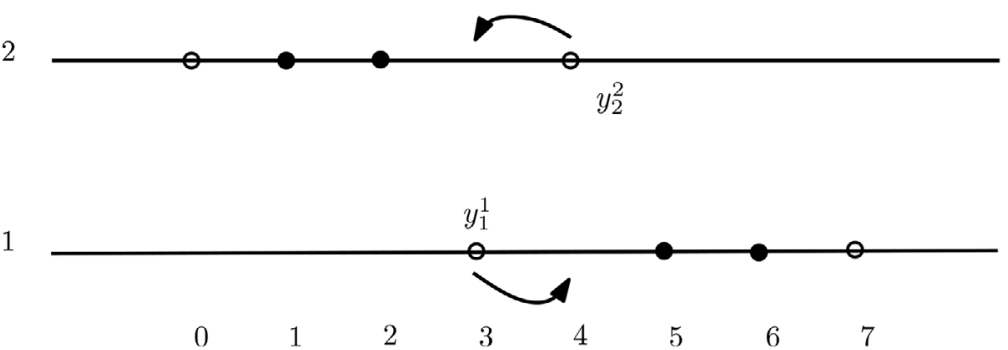

FIGURE 7

$v_{1}^{c}=y_{1}^{1} ;$ AN FIP IS POSSIBLE

Proposition 3. Let $\mathbf{x}$ and $\mathbf{y}$ be two distributions in $\mathcal{D}(I)$ such that $\mathbf{x} \succsim^{O P G} \mathbf{y}$. Suppose that $v_{1}^{c}=y_{1}^{1}$. Then there exists a distribution $\widehat{\mathbf{x}} \in \mathcal{D}(I)$ such that $\widehat{\mathbf{x}}$ is obtained from $\mathbf{y}$ by an FIP and $\mathbf{x} \succsim^{O P G} \widehat{\mathbf{x}}$.

An illustration of Proposition 3 can be provided in the case where $k=2$ and $\mathcal{N}(1)=\mathcal{N}(2)=$ $\{1,2\}$ by considering the distributions $\mathbf{y}$ and $\mathbf{x}$ defined, respectively, by:

$$
y_{1}^{1}=3, y_{2}^{1}=7 y_{1}^{2}=0, y_{2}^{2}=4
$$

and:

$$
x_{1}^{1}=5, x_{2}^{1}=6, x_{1}^{2}=1, x_{2}^{2}=2
$$

It can be verified that $\mathbf{x} \succsim^{O P G} \mathbf{y}$ and $v_{1}^{c}=3=y_{1}^{1}$. One can see (Figure 7) that agent $i_{2}=2$ can exchange his income with agent 1 in category 1 without breaking any of the OPG inequalities.

3.2. Proof of the Main Result (iii) $\Rightarrow(i)$. We now use the diagnostic tool introduced in the preceding subsection to prove the last implication of Theorem 1. For this sake, we let $\mathbf{x}$ and $\mathbf{y}$ be two distributions that are as specified in Theorem 1. By a recursive argument on the finite set of agents, proving the main implication of Theorem 1 amounts to showing that $\mathbf{x} \succsim^{O P G} \mathbf{y}$ implies the possibility of going from $\mathbf{y}$ to some distribution $\overline{\mathbf{x}} \in \mathcal{D}(I)$ by a finite sequence of BTPIT and/or FIP in such a way that:

- $\mathbf{x}{ }^{O P G} \overline{\mathbf{x}}$,

- there exists $h \in\{1, \ldots, k\}$ for which $x_{i}^{h}=\bar{x}_{\bar{i}}^{h}$ for some $i$ and $\bar{i} \in \mathcal{N}(h)$.

Indeed, whenever one agent in one category has reached the income level that an agent of this category has in final distribution $\mathbf{x}$, we can remove that agent from that category and restart the procedure. Since the numbers of agents and categories are finite, this completes the proof. In order to construct our algorithm for moving from $\mathbf{y}$ to some distribution $\overline{\mathbf{x}}$ as described above by a finite sequence of BTPIT and/or FIP, we first set $\mathbf{x}(0):=\mathbf{y}$ and we recursively define $\mathbf{x}(n+1)$ from $\mathbf{x}(n)$ through the following procedure, after defining $v_{1}^{c}(n)$ to be the critical value defined as per (9) but applied to $\mathbf{x}(n)$ instead of to $\mathbf{y}$.

$(P 1)$ If $v_{1}^{c}(n)=x_{1}^{1}(n)$ then perform an FIP, which is possible according to Proposition 3 .

$(P 2)$ If $v_{1}^{c}(n)>x_{1}^{1}(n)$ and if $\forall i \in \mathcal{N}(1), x_{i}^{1}>v_{1}^{c}(n)$ and for any category $h$ such that $h_{0} \geq$ $h \geq 2$, we have $x_{i}^{h}(n) \neq v_{1}^{c}(n)$ where $h_{0}$ is the category such that $v_{1}^{c}(n)=v_{h_{0}}^{c}(n)>$ $v_{h_{0}+1}^{c}(n)$, then perform an FIP, as described by Proposition 2 (remembering that the recipient of such an FIP is not the poorest individual in category 1 in that case).

$(M T)$ Otherwise perform the maximal transfer defined by Proposition 1 and Definition 6. 
By construction, $\mathbf{x} \succsim^{O P G} \mathbf{x}(n)$ for any $n$. If there exists some $n^{*} \in \mathbb{N}_{+}$such that, for some category $h \in\{1, \ldots, k\}$, we have:

$$
x_{i}^{h}\left(n^{*}\right)=x_{j}^{h}
$$

for some $i, j \in \mathcal{N}(h)$ then the algorithm ends and is said to be finite. If it does not end, then the algorithm generates an infinite (nonstationary) sequence. The only thing that remains to be proved (Subsection 6.7 of the Appendix) is that the latter is impossible and that the algorithm is indeed finite.

The danger of falling into an endless sequence of transfers starting from an OPGdominated distribution $\mathbf{y}$ and going to a dominating distribution $\mathbf{x}$ that we exclude by our algorithmic procedure is a real one. It is the concern for avoiding this danger that makes us choose an FIP in any step of the algorithm labeled as $(P 2)$ above, even though performing a BTPIT transfer would also be possible in that case under Proposition 1. In order to illustrate the danger, consider again the example discussed after Proposition 2 where $k=2, \mathcal{N}(1)=$ $\{1,2,3\}, \mathcal{N}(2)=\{1,2,3,4\}$ and $\mathbf{y}$ and $\mathbf{x}$ are defined by:

$$
y_{1}^{1}=2, y_{2}^{1}=y_{3}^{1}=3, y_{1}^{2}=0, y_{2}^{2}=y_{3}^{2}=y_{4}^{2}=4
$$

and:

$$
x_{1}^{1}=x_{2}^{1}=x_{3}^{1}=4, x_{1}^{2}=x_{2}^{2}=x_{3}^{2}=x_{4}^{2}=2 .
$$

If a BTPIT instead of an FIP were performed in this case, the maximal transfer would clearly be a half transfer of $1 / 2$. Performing this transfer would yield the distribution $\widehat{\mathbf{x}}$ defined by:

$$
\widehat{x}_{1}^{1}=\widehat{x}_{2}^{1}=5 / 2, \widehat{x}_{3}^{1}=3, \widehat{x}_{1}^{2}=0, \widehat{x}_{2}^{2}=\widehat{x}_{3}^{2}=\widehat{x}_{4}^{2}=4 .
$$

Note that the critical value $v_{1}^{c}(\widehat{\mathbf{x}})$ associated with $\widehat{\mathbf{x}}$ is still $3>\widehat{x}_{1}^{1}=5 / 2$. Proposition 1 indicates that agent 3 in category 1 can make a transfer to one of the two poorest agents in that same category. The maximal transfer that agent 3 of category 1 can transfer to either one of the two poorest agents of category 1 is a half transfer of $1 / 4$. If this transfer is performed, then distribution $\widehat{\widehat{\mathbf{x}}}$ is obtained, with $\widehat{\widehat{\mathbf{x}}}$ defined by:

$$
\widehat{\widehat{x}}_{1}^{1}=5 / 2, \widehat{\hat{x}}_{2}^{1}=\widehat{\hat{x}}_{3}^{1}=11 / 4, \widehat{\widehat{x}}_{1}^{2}=0, \widehat{\widehat{x}}_{2}^{2}=\widehat{\widehat{x}}_{3}^{2}=\widehat{\widehat{x}}_{4}^{2}=4
$$

However, from this distribution $\widehat{\widehat{\mathbf{x}}}$, the critical value $v_{1}^{c}(\widehat{\mathbf{x}})$ is $11 / 4$ and this would have allowed a half transfer of $1 / 8$ between either agent 2 or 3 in category 1 and the poorest agent 1 in this category and so on. Systematically resorting to the transfer allowed by Proposition 1 in that case would generate an infinite sequence of half transfers (with the "half" becoming smaller and smaller). Choosing an FIP instead of a BTPIT in the situation described by Proposition 2 enables us to avoid falling into the trap of an infinite sequence of shrinking transfers.

\section{EXTENSIONS}

The analysis of the two previous sections was restricted to distributions with the same number of agents in every category and the same total income. This restriction was motivated by our objective of defining pure inequality reduction among unequals. We now indicate how the analysis can be extended when the restrictions are removed. We first discuss the case where the number of agents in each category is allowed to vary across distributions, keeping constant both the total number of agents, $n$, and the total income to be distributed. We then indicate how these two last restrictions can also be removed. 
Denote by $\mathcal{N}_{\mathbf{x}}(h)$ the (now possibly empty) set of agents in category $h$ for distribution $\mathbf{x}$ and by $n_{\mathbf{x}}(h)=\# \mathcal{N}_{\mathbf{x}}(h)$ the number of those agents. We write $\mathbf{x}=\left\{\left(x_{1}^{h}, \ldots, x_{n_{\mathbf{x}}(h)}^{h}\right)\right\}_{h=1}^{k}$ with the convention that, for every $h,\left\{\left(x_{1}^{h}, \ldots, x_{n_{\mathbf{x}}(h)}^{h}\right)\right\}=\varnothing$ if $n_{\mathbf{x}}(h)=0$. As mentioned, we maintain for the moment the assumption that:

$$
\sum_{h=1}^{k} n_{\mathbf{x}}(h)=n ; \sum_{h=1}^{k} \sum_{i=1}^{n_{\mathbf{x}}(h)} x_{i}^{h}=I
$$

We call $\overline{\mathcal{D}}(I)$ the set of all such distributions. In order to allow for the number of agents in a given category to vary across distributions, we introduce the following elementary operation, that will be added to the FIP and BTPIT discussed earlier.

Definition 7 (CATEgorical increments). Let $\mathbf{x}$ and $\mathbf{y}$ be two distributions in $\overline{\mathcal{D}}(I)$. We say that $\mathbf{x}$ is obtained from $\mathbf{y}$ by means of a categorical increment if there are categories $g$ and $h$ for which $g<h, n_{\mathbf{x}}(g)=n_{\mathbf{y}}(g)-1, n_{\mathbf{x}}(h)=n_{\mathbf{y}}(h)+1$, as well as $i^{g} \in \mathcal{N}_{\mathbf{y}}(g)$ and $i^{h} \in \mathcal{N}_{\mathbf{x}}(h)$ such that:

(i) $x_{i}^{g}=y_{i+1}^{g}$ for all $i \geq i^{g}$ (if any).

(ii) $x_{i h}^{h}=y_{i g}^{g}$.

(iii) $x_{i}^{h}=y_{i-1}^{h}$ for all $i \geq i^{h}+1$ (if any)

(iv) $x_{i}^{l}=y_{i}^{l}$ for any other pair $(i, l)$.

In words, $\mathbf{x}$ is obtained from $\mathbf{y}$ by means of a categorical increment if the only difference between $\mathbf{x}$ and $\mathbf{y}$ is that one agent in some category in $\mathbf{y}$ moves up a higher category in $\mathbf{x}$ while keeping his/her income. The following result extends Theorem 1 to this new setting. This extension requires some modification of the notations and the definitions. In particular, the class of utility functions over which utilitarian unanimity is now sought has to be restricted to those utility functions that are increasing with respect to the agent's type. This restricted class is denoted by $\mathcal{U}^{* *}$ and contains all lists of type-dependent $\left(U^{1}, \ldots, U^{k}\right) \in \mathcal{U}^{*}$ that satisfy, in addition, the property that:

$$
U^{h^{\prime}}(w) \geq U^{h}(w) \forall h^{\prime} \geq h,
$$

and for every $w$. We can now establish the following Theorem.

Theorem 2. Let $\mathbf{x}$ and $\mathbf{y}$ be two distributions in $\overline{\mathcal{D}}(I)$. Then the following four statements are equivalent:

(i) It is possible to go from $\mathbf{y}$ to $\mathbf{x}$ by a finite sequence of categorical increments, BTPIT and/or FIP,

(ii) $\mathbf{x}$ utilitarian dominates $\mathbf{y}$ for the class $\mathcal{U}^{* *}$,

(iii) $\mathbf{x} \succsim^{O P G} \mathbf{y}$ :

$$
\sum_{h=1}^{k} \sum_{i=1}^{n_{\mathbf{x}}(h)} \max \left(v_{h}-x_{i}^{h}, 0\right) \leq \sum_{h=1}^{k} \sum_{i=1}^{n_{\mathbf{y}}(h)} \max \left(v_{h}-y_{i}^{h}, 0\right), \quad \forall\left(v_{1}, \ldots, v_{k}\right) \in \mathcal{V}
$$

(iv) $\mathbf{x} \succsim^{C L I} \mathbf{y}$, where the set of eligible definitions of the $m$ poorest agents now writes

$$
\Pi(\mathbf{x}, m)=\left\{\left(r_{1}, \ldots, r_{k}\right): 0 \leq r_{h} \leq n_{\mathbf{x}}(h), \sum_{h=1}^{k} r_{h}=m, x_{r_{h}+1}^{h}>x_{r_{h^{\prime}}}^{h^{\prime}} \forall h^{\prime}>h\right\}
$$


REMARK 1. If $\mathbf{x}$ dominates $\mathbf{y}$ with respect to any of the four equivalent statements of Theorem 2 , then we necessarily have

$$
\sum_{h=1}^{g} n_{\mathbf{y}}(h) \geq \sum_{h=1}^{g} n_{\mathbf{x}}(h), \quad \forall g=1, \ldots, k
$$

Hence, first-order stochastic dominance of its marginal distribution of needs is a necessary condition for $\mathbf{x}$ to dominate $\mathbf{y}$ for any of the four equivalent statements of Theorem 2 .

REMARK 2. When the number of agents in the different categories is allowed to vary across distributions, OPG dominance is not equivalent to inequality (12) for lists of ordered poverty lines of the subset $\mathcal{V}^{\prime}$ defined in (A.17). Consider the following simple example involving 2 categories and 2 agents (all in category 1 in distribution $\mathbf{x}$ and split between the two categories in distribution $\mathbf{y}$ ). Incomes are: $x_{1}^{1}=x_{2}^{1}=1$ and $y_{1}^{1}=0, y_{1}^{2}=2$. Then it is easy to check that $\mathbf{x}$ OPG-dominates $\mathbf{y}$ if we restrict the poverty lines to $2 \geq v_{1} \geq v_{2} \geq 0$. Nevertheless $\mathbf{x}$ does not dominate $\mathbf{y}$ for the criteria $(i),(i i)$ and (iv). This nondominance can be seen by considering, say, a poverty line of 3 in category 1 and 0 in category 2 .

We stress that although Theorem 2 extends Theorem 1 to distributions with varying distributions of $n$ agents in the $k$ categories, it does so only by recognizing the categorical increment as an appealing elementary operation. Although this intuitive appeal may be obvious in contexts where the nonpecuniary source of differentiation of the agents is a clear positive contributor to the agent's welfare, such as health or education, it may not be in others. As mentioned earlier, if agents are households without children that are divided between single persons and couples, not everyone would consider a reduction in the fraction of couples in the population as a significant social improvement. Furthermore, a categorical increment can hardly be considered to be an "equalizing operation." It is more about improving efficiency than improving equality.

We now indicate how the results can be extended to the case where $n$ (the total number of agents) is the same but where the total income $I$ to be distributed varies. To do so, we let $\overline{\mathcal{D}}=\bigcup_{I \in \mathbb{R}++} \overline{\mathcal{D}}(I)$ denote the set of all income distributions $\mathbf{x}=\left\{\left(x_{1}^{h}, \ldots, x_{n_{\mathbf{x}}(h)}^{h}\right)\right\}_{h=1}^{k}$ such that $\sum_{h=1}^{k} \sum_{i=1}^{n_{\mathbf{x}}(h)} x_{i}^{h}=I_{\mathbf{x}}$ for some strictly positive real number $I_{\mathbf{x}}$ and list of integers $\left(n_{\mathbf{x}}(1), \ldots, n_{\mathbf{x}}(k)\right) \in$ $\{0, \ldots, n\}^{k}$ (both depending upon the distribution) satisfying $n_{\mathbf{x}}(1)+\cdots+n_{\mathbf{x}}(k)=n$.

There are at least two ways of extending Theorems 1 and 2 to this domain of distributions with varying total income.

The first way would be to consider, in line with the literature on the measurement of relative inequality, a "scale-independance" property (see e.g., Cowell (2011)), that makes the multiplication of the $n$ agents' incomes by any common "scaling" factor a matter of indifference. Such a property could be stated formally as follows.

Axiom 1 (Scale Independence). If $\mathbf{x}$ and $\mathbf{y}$ are two income distributions in $\overline{\mathcal{D}}$ such that, for all $h=1, \ldots, k,\left(x_{1}^{h}, \ldots, x_{n_{\mathbf{x}}(h)}^{h}\right)=\left(\lambda y_{1}^{h}, \ldots, \lambda y_{n_{y}(h)}^{h}\right)$ for some strictly positive real number $\lambda$, then $\mathbf{x}$ and $\mathbf{y}$ are indistinguishable from the viewpoint of inequality.

If one accepts such an axiom-there are many reasons not to-then one can take any income distribution $\mathbf{x}$ in $\overline{\mathcal{D}}$ and make it indistinguishable, from the viewpoint of inequality, from its total income share equivalent $\mathbf{x}^{s} \in \overline{\mathcal{D}}(1)$ defined by:

$$
\left(x_{1}^{h}, \ldots, x_{n_{\mathbf{x}}(h)}^{h}\right)=\left(x_{1}^{h} / I_{\mathbf{x}}, \ldots, y_{n_{x}(h)}^{h} / I_{\mathbf{x}}\right)
$$


for all $h=1, \ldots, k$ and for $\sum_{h=1}^{k} \sum_{i=1}^{n_{\mathbf{x}}(h)} x_{i}^{h}=I_{\mathbf{x}}$. It then becomes possible to rank any two distributions $\mathbf{x}$ and $\mathbf{y}$ in $\overline{\mathcal{D}}$ as per the ranking of their total income share equivalent $\mathbf{x}^{s}$ and $\mathbf{y}^{s}$ obtained on the basis of applying either Theorem 1 to the set $\mathcal{D}(1)$ (in the case where the set of agents belonging to every category is the same in $\mathbf{x}$ and $\mathbf{y}$ ) or Theorem 2 to the set $\overline{\mathcal{D}}(1)$ (in the other case).

Another approach to comparing distributions with a variable total income would be to follow Shorrocks (1983) and to recognize the social value of increasing the income of some, or all, agents. The standard way of doing this is to introduce another elementary transformation, which we call income increment and which we define as follows:

Definition 8 (InCOME InCREMENTs). Let $\mathbf{x}$ and $\mathbf{y}$ be two distributions in $\overline{\mathcal{D}}$. We say that $\mathbf{x}$ is obtained from $\mathbf{y}$ by means of an income increment if $\mathcal{N}_{\mathbf{x}}(g)=\mathcal{N}_{\mathbf{y}}(g)$ for any $g$, and there exists a category $h, i^{h} \leq j^{h} \in \mathcal{N}(h)$ and $a>0$ such that:

(i) $x_{i}^{h}=y_{i+1}^{h}$ for $i=i^{h} \ldots j^{h}-1$ (if any).

(ii) $x_{j_{h}}^{h}=y_{i_{h}}^{h}+a$.

(iii) $x_{i}^{l}=y_{i}^{l}$ for any other pair $(i, l)$.

In words, $\mathbf{x}$ is obtained from $\mathbf{y}$ by means of an income increment if $\mathbf{x}$ and $\mathbf{y}$ only differ by the fact that one agent in some category has an higher income in distribution $\mathbf{x}$ than in distribution $\mathbf{y}$. Recognizing the value of increasing the income of some agent by $a$ dollars in a utilitarian (or even more general welfarist) perspective obviously requires that the set of type-dependent utility functions considered by this perspective be restricted to those lists $\left(U^{1}, \ldots, U^{k}\right) \in \mathcal{U}^{* *}$ satisfying the following additional property:

$$
U^{h}(w+a) \geq U^{h}(w) \forall h, \forall a \geq 0 .
$$

Denote by $\mathcal{U}^{* * *}$ this set. We can then state the following theorem whose proof can easily be worked out using standard results in one-dimensional dominance analysis-for instance, by combining Quirk and Saposnik (1962) and Shorrocks (1983) — and the fact that the proof of the equivalence between (iii) and (iv) in Theorem 1 does not assume anything about the sum of income that is distributed. The proof is omitted here.

TheOrem 3. Let $\mathbf{x}$ and $\mathbf{y}$ be two distributions in $\overline{\mathcal{D}}$. Then the following four statements are equivalent.

(i) It is possible to go from $\mathbf{y}$ to $\mathbf{x}$ by a finite sequence of income increments, categorical increments, BTPIT and/or FIP.

(ii) $\mathbf{x}$ utilitarian dominates $\mathbf{y}$ for the class $\mathcal{U}^{* * *}$.

(iii) $\mathbf{x} \succsim^{O P G} \mathbf{y}$.

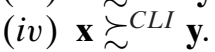

Of course, we could provide a similar theorem for the case where the two distributions of Theorem 3 have the same set of agents in every category by simply removing the categorical increments from the list of elementary transformations in Statement (i), and by considering instead of $\mathcal{U}^{* * *}$ in Statement (ii) the set of lists of type-dependent utility functions in $\left(U^{1}, \ldots, U^{k}\right) \in \mathcal{U}^{*}$ that satisfy Inequality (13).

Finally, the case where the total number of agents varies across distributions is handled by applying the so-called Dalton principle of population replication (see, e.g., Dalton, 1920). In the present setting, this principle says that replicating any finite number of times a distribution of income among agents in different categories is a matter of social indifference. 
Formally, consider a distribution $\mathbf{x}=\left\{\left(x_{1}^{h}, \ldots, x_{n_{\mathbf{x}}(h)}^{h}\right)\right\}_{h=1}^{k}$ with $\sum_{h=1}^{k} \sum_{i=1}^{n_{\mathbf{x}}(h)} x_{i}^{h}=I_{\mathbf{x}}$ for some strictly positive real number $I_{\mathbf{x}}$ and list of integers $\left(n_{\mathbf{x}}(1), \ldots, n_{\mathbf{x}}(k)\right) \in\{0, \ldots, n\}^{k}$ satisfying $n_{\mathbf{x}}(1)+$ $\cdots+n_{\mathbf{x}}(k)=n_{\mathbf{x}}$ for some strictly positive (but now distribution-dependent) integer $n_{\mathbf{x}}$. For any strictly positive integer $m$, one can then define the $m$-replicate of distribution $\mathbf{x}$, denoted $\mathbf{x}^{m}$, by:

$$
\mathbf{x}^{m}=\left\{(\underbrace{x_{1}^{h}, x_{1}^{h}, \ldots, x_{1}^{h}}_{\mathrm{m} \text {-times }}, \ldots, \underbrace{x_{n_{\mathbf{x}}(h)}^{h}, x_{n_{\mathbf{x}}(h)}^{h}, \ldots, x_{n_{\mathbf{x}}(h)}^{h}}_{\mathrm{m} \text {-times }})\right\}_{h=1}^{k} .
$$

Observe that the $m$-replicate of distribution $\mathbf{x}$ in which there are $n$ agents is a distribution in which there are $n m$ agents. If one adopts the principle that any distribution $\mathbf{x}$ is indistinguishable, from the viewpoint of inequality, from its $m$-replicate $\mathbf{x}^{m}$ for any $m$, then one can compare the distribution $\mathbf{x}$ with $n$ agents to the distribution $\mathbf{y}$ with $m$ agents exactly in the same way as one would compare the $m$-replicate of $\mathbf{x}$ to the $n$-replicate of $\mathbf{y}$ The comparison of the two distributions $\mathbf{x}^{m}$ and $\mathbf{y}^{n}$ (both with the same number $n m$ of agents) could then be made on the basis of any of the criteria indicated in Theorems 1-3.

\section{CONCLUSION}

In this article, we provide a workable definition of "income equalization" between agents who are vertically differentiated with respect to some other characteristic. Such equalization is defined as the transfer from a richer and more highly ranked agent to a poorer and less highly ranked agent of an amount of income that does not exceed the income difference between the two agents. If the transfer does not exceed half the income difference between the donor and the receiver, it is called a BTPIT. If the transfer is larger than half the income difference, it can be viewed as a combination of a BTPIT of less than half the income difference and an FIP. The article has identified the normative foundations of this notion of equalization. Specifically, it has shown that the smallest transitive ranking of distributions consistent with this notion of equalization is the unanimity of all utilitarian planners' rankings considering that the marginal utility of income for every agent is decreasing with respect to both income and type. The article has also identified two empirically implementable criteria-the OPG criterion, and the CLI criterion - that are both equivalent to this notion of equalization. Although Gravel and Moyes (2012) showed that the OPG dominance of one distribution over another is equivalent to the possibility of going from a phantom-augmented dominated distribution to a phantomaugmented dominating one by a finite sequence of Pigou-Dalton transfers (between agents of a given type) and/or FIP, these authors could not establish the equivalence without resorting to dummy or phantom agents. The present article is therefore, to the best of our knowledge, the first to provide an equivalence between a notion of normative dominance, an elementary notion of equalization, and two empirically implementable criteria that applies to distributions of a cardinally meaningful attribute among vertically differentiated agents.

There are at least three directions in which the analysis of this article could be extended. One of them concerns empirical applications of the dominance criteria. The OPG dominance criterion has not been widely used in empirical analysis, even though some authors (e.g., Gravel et al., 2009) have shown that it is amenable to statistical inference techniques of the kind suggested by Davidson and Duclos (2000). It is our hope that the arguments provided in its - or in that of its CLI counterpart - will encourage its use in empirical work. Another direction for future research would be to better appraise the performance of the two implementable criteria - OPG and CLI dominance-in terms of computational complexity. The third direction for future research would be to identify inequality indices compatible with the incomplete ranking of distribution characterized herein. 


\section{A APPENDIX}

A.1 Formal Definitions of Section 2. We here provide the rigorously perfect definitions of BTPIT and FIP that allow the rank of the agents in their categories' income distributions to be affected by the elementary transformations.

BTPIT: A rigorous definition. Let $\mathbf{x}$ and $\mathbf{y}$ be two distributions in $\mathcal{D}(I)$. We say that $\mathbf{x}$ is obtained from $\mathbf{y}$ by means of a BTPIT if there are categories $g$ and $h$ for which $g \leq h$, two agents $i^{g} \in \mathcal{N}(g)$ and $i^{h} \in \mathcal{N}(h)$ for which $y_{i^{h}}^{h}>y_{i^{g}}^{g}$ and a number $\left.\left.\alpha \in\right] 0, \frac{y_{h^{h}}^{h}-y_{i^{g}}^{g}}{2}\right]$ such that:

(i) $x_{i}^{g}=y_{i+1}^{g}$ for all $i \in \mathcal{N}(g)$ such that $i^{g} \leq i<r_{+}^{g}(\alpha)$ (if any).

(ii) $x_{r_{+}^{g}(\alpha)}^{g}=y_{i^{g}}^{g}+\alpha$.

(iii) $x_{i}^{h}=y_{i-1}^{h}$ for all $i \in \mathcal{N}(h)$ such that $r_{-}^{h}(\alpha)<i \leq i^{h}$ (if any)

(iv) $x_{r_{-}^{h}(\alpha)}^{h}=y_{i^{h}}^{h}-\alpha$.

(v) $x_{i}^{l}=y_{i}^{l}$ for any other pair $(i, l)$ where $l \in\{1, \ldots, k\}$ and $i \in \mathcal{N}(l)$,

where $r_{+}^{g}(\alpha):=\max \left\{i \in \mathcal{N}(g): y_{i}^{g}<y_{i^{g}}^{g}+\alpha\right\}, r_{-}^{h}(\alpha):=\min \left\{i \in \mathcal{N}(h): y_{i}^{h}>y_{i^{h}}^{h}-\alpha\right\}$.

FIP: A rigorous definition. Let $\mathbf{x}$ and $\mathbf{y}$ be two distributions in $\mathcal{D}(I)$. We say that $\mathbf{x}$ is obtained from $\mathbf{y}$ by means of an FIP if there are categories $g$ and $h$ for which $g<h$ and two agents $i^{g} \in \mathcal{N}(g)$ and $i^{h} \in \mathcal{N}(h)$ for which $y_{i^{h}}^{h}>y_{i^{g}}^{g}$ such that:

(i) $x_{i}^{g}=y_{i+1}^{g}$ for all $i \in \mathcal{N}(g)$ such that $i^{g} \leq i<r^{g}\left(i^{h}\right)$ (if any).

(ii) $x_{r^{g}\left(i^{h}\right)}^{g}=y_{i^{h}}^{h}$.

(iii) $x_{i}^{h}=y_{i-1}^{h}$ for all $i \in \mathcal{N}(h)$ such that $r^{h}\left(i^{g}\right)<i \leq i^{h}$ (if any).

(iv) $x_{r^{h}\left(i^{g}\right)}^{h}=y_{i^{\circ}}^{g}$.

(v) $x_{i}^{l}=y_{i}^{l}$ for any other pair $(i, l)$ where $l \in\{1, \ldots, k\}$ and $i \in \mathcal{N}(l)$,

where $r^{g}\left(i^{h}\right):=\max \left\{i \in \mathcal{N}(g): y_{i}^{g}<y_{i^{h}}^{h}\right\}$ and $r^{h}\left(i^{g}\right):=\min \left\{i \in \mathcal{N}(h): y_{i}^{h}>y_{i^{g}}^{g}\right\}$.

\section{A.2 Proof of $(i) \Rightarrow(i i) \Rightarrow($ iii $)$.}

Proposition A.1. Let $\mathbf{x}$ and $\mathbf{y}$ be two distributions in $\mathcal{D}(I)$. Then, in Theorem 1, Statement (i) implies statement (ii) and statement (ii) implies statement (iii).

Proof. $(i) \Rightarrow$ (ii) We must prove that both BTPIT and FIP increase the sum of utilities for any collection of utility functions $\left\{U^{h}\right\}_{h=1}^{k} \in \mathcal{U}^{*}$.

BTPIT: Assume that $\mathbf{x}$ is obtained from $\mathbf{y}$ by a BTPIT. Then, using Definition 1, there are categories $g$ and $h$ satisfying $g \leq h$, agents $i^{g} \in \mathcal{N}(g)$ and $i^{h} \in \mathcal{N}(h)$ satisfying $y_{i^{g}}^{g}<y_{i^{h}}^{h}$ and a number $\alpha \in\left[0,\left(y_{i^{h}}^{h}-y_{i^{g}}^{g}\right) / 2\right]$ for which we have:

$$
\begin{aligned}
& \sum_{j=1}^{k} \sum_{i=1}^{n(j)}\left(U^{j}\left(x_{i}^{j}\right)-U^{j}\left(y_{i}^{j}\right)\right) \\
= & U^{g}\left(x_{r_{+}^{g}(\alpha)}^{g}\right)-U^{g}\left(y_{i^{g}}^{g}\right)+U^{h}\left(x_{r_{-}^{h}(\alpha)}^{h}\right)-U^{h}\left(y_{i^{h}}^{h}\right) \\
= & U^{g}\left(y_{i^{g}}^{g}+\alpha\right)-U^{g}\left(y_{i^{g}}^{g}\right)-\left[U^{h}\left(y_{i^{h}}^{h}\right)-U^{h}\left(y_{i^{h}}^{h}-\alpha\right)\right] \\
\geq & \left.0 \text { (if the functions } U^{1}, \ldots, U^{k} \text { belong to } \mathcal{U}^{*}\right) .
\end{aligned}
$$

FIP: Assume that $\mathbf{x}$ is obtained from $\mathbf{y}$ by an FIP. Then, using Definition 2, there are categories $g$ and $h$ satisfying $g<h$, agents $i^{g} \in \mathcal{N}(g)$ and $i^{h} \in \mathcal{N}(h)$ satisfying $y_{i^{g}}^{g}<y_{i^{h}}^{h}$ for which we 
have:

$$
\begin{aligned}
\sum_{j=1}^{k} \sum_{i=1}^{n(j)}\left(U^{j}\left(x_{i}^{j}\right)-U^{j}\left(y_{i}^{j}\right)\right) & =U^{g}\left(x_{r^{g}\left(i^{h}\right)}^{g}\right)-U^{g}\left(y_{i^{g}}^{g}\right)+U^{h}\left(x_{r^{h}\left(i^{g}\right)}^{h}\right)-U^{h}\left(y_{i^{h}}^{h}\right) \\
& =U^{g}\left(y_{i^{h}}^{h}\right)-U^{g}\left(y_{i^{g}}^{g}\right)+U^{h}\left(y_{i^{g}}^{g}\right)-U^{h}\left(y_{i^{h}}^{h}\right) \\
& =U^{g}\left(y_{i^{h}}^{h}\right)-U^{g}\left(y_{i^{g}}^{g}\right)-\left[U^{h}\left(y_{i^{h}}^{h}\right)-U^{h}\left(y_{i^{g}}^{g}\right)\right] \\
& \left.\geq 0 \text { if the functions } U^{1}, \ldots, U^{k} \text { belong to } \mathcal{U}^{*}\right) .
\end{aligned}
$$

Repeating the arguments (for the FIP and/or the BTPIT) for any finite sequence of distributions of income completes the proof of the first implication for the theorem.

$(i i) \Rightarrow(i i i)$. Let $\mathbf{x}$ and $\mathbf{y}$ be two distributions in $\mathcal{D}(I)$ for which the inequality:

$$
\sum_{h=1}^{k} \sum_{i=1}^{n(t)} U^{h}\left(x_{i}^{h}\right)-\sum_{h=1}^{k} \sum_{i=1}^{n(t)} U^{h}\left(y_{i}^{h}\right) \geq 0
$$

holds for all lists of utility functions $\left\{U^{h}\right\}_{h=1}^{k}$ in $\mathcal{U}^{*}$. Choose any vector $\mathbf{v}=\left(v_{1}, \ldots, v_{k}\right)$ in the set $\mathcal{V}$ and define the $k$ functions $U^{h}: \mathbb{R} \longrightarrow \mathbb{R}$ (for $h=1, \ldots, k$ ) by $U^{h}(w)=\min \left[w-v_{h}, 0\right]$. Let us show that the collection of $k$ functions $\left\{U^{h}\right\}_{h=1, \ldots, k}$ satisfies inequality (3) for any vector $\mathbf{v}=\left(v_{1}, \ldots, v_{k}\right)$ in $\mathcal{V}$, and therefore belongs to $\mathcal{U}^{*}$. Consider any $u \geq 0, w \leq w^{\prime}$ and $h \leq h^{\prime}$. First note that the quantities $U^{h}(w+u)-U^{h}(w)$ and $U^{h^{\prime}}\left(w^{\prime}+u\right)-U^{h^{\prime}}\left(w^{\prime}\right)$ belong to [0, $u$.

If $w \geq v_{h}$ then $w+u \geq v_{h}$ and $w^{\prime}+u \geq w^{\prime} \geq v_{h^{\prime}}$. Thus, (3) holds with both sides equal to zero.

If $w \leq v_{h}$ then $U^{h}(w)=w-v_{h}$ and $U^{h}(w+u)-U^{h}(w)=\min \left(u, v_{h}-w\right)$. Note also that:

$$
U^{h^{\prime}}\left(w^{\prime}+u\right)-U^{h^{\prime}}\left(w^{\prime}\right) \leq-U^{h^{\prime}}\left(w^{\prime}\right) \leq v_{h^{\prime}}-w^{\prime} \leq v_{h}-w .
$$

Hence, $U^{h^{\prime}}\left(w^{\prime}+u\right)-U^{h^{\prime}}\left(w^{\prime}\right) \leq \min \left(u, v_{h}-w\right)$ and inequality (3) holds for that case also. We have therefore proved that the collection of functions $\left\{U^{h}\right\}_{h=1, \ldots, k}$ belongs to the class $\mathcal{U}^{*}$ for all $\mathbf{v}=\left(v_{1}, \ldots, v_{k}\right) \in \mathcal{V}$. Since inequality (A.1) holds for all such functions so that we have:

$$
\begin{aligned}
\sum_{h=1}^{k} \sum_{i=1}^{n(h)} \min \left[x_{i}^{h}-v_{h}, 0\right] & \geq \sum_{h=1}^{k} \sum_{i=1}^{n(h)} \min \left[y_{i}^{h}-v_{h}, 0\right] \\
& \Longleftrightarrow \\
\sum_{h=1}^{k} \sum_{i=1}^{n(h)} \max \left[v_{h}-x_{i}^{h}, 0\right] & \leq \sum_{h=1}^{k} \sum_{i=1}^{n(h)} \max \left[v_{h}-y_{i}^{h}, 0\right]
\end{aligned}
$$

for all $\mathbf{v}=\left(v_{1}, \ldots, v_{k}\right) \in \mathcal{V}$, as required by the OPG criterion.

A.3 Proof of $($ iii $) \Leftrightarrow(i v)$.

Proposition A.2. Let $\mathbf{x}$ and $\mathbf{y}$ be two distributions in $\mathcal{D}(I)$. Then $\mathbf{x} \succsim^{O P G} \mathbf{y}$ if and only if $\mathbf{x} \succsim^{C L I} \mathbf{y}$.

Proof. Assume first that $\mathbf{x} \succsim^{C L I} \mathbf{y}$, and consider any $\mathbf{v} \in \mathcal{V}$. We must show that $P^{\mathbf{x}}(\mathbf{v}) \leq$ $P^{\mathbf{y}}(\mathbf{v})$. Let the vector of ranks $\left(\widetilde{r_{1}}, \ldots, r_{k}\right)$ be defined, for any $h \in\{1, \ldots, k\}$ by:

$$
\begin{aligned}
r_{h} & =\max \left\{i \in \mathcal{N}(h): x_{i}^{h} \leq v_{h}\right\}, \text { if }\left\{i \in \mathcal{N}(h): x_{i}^{h} \leq v_{h}\right\} \neq \varnothing \\
& =0 \text { otherwise. }
\end{aligned}
$$


Clearly, $r_{h} \in\left\{0, \ldots, n_{h}\right\}$ for any $h$. Let $m=\sum_{h} r_{h}$. We first show that $\left(r_{1}, \ldots, r_{k}\right) \in \Pi(\mathbf{x}, m)$. By contradiction, assume that $\left(r_{1}, \ldots, r_{k}\right) \notin \Pi(\mathbf{x}, m)$. By definition of $\Pi(\mathbf{x}, m)$ (Expression (6)), there exist categories $h$ and $h^{\prime}$ satisfying $h^{\prime}>h$ such that $x_{r_{h}+1}^{h} \leq x_{r_{h^{\prime}}}^{h^{\prime}}$. By definition of $r_{h}$, we have that $x_{r_{h^{\prime}}}^{h^{\prime}} \geq x_{r_{h}+1}^{h}>x_{r_{h}}^{h} \geq \underline{v}(\mathbf{x}, \mathbf{y})$ (at least under the convention that $x_{0}^{h}=\underline{v}(\mathbf{x}, \mathbf{y})$ ). This means that $x_{r_{h^{\prime}}}^{h^{\prime}}=v^{h^{\prime}}>v^{h}=x_{r_{h}}^{h}$ (if $r_{h} \geq 1$ ) and $x_{r_{h^{\prime}}}^{h^{\prime}}=v^{h^{\prime}}>v^{h}$ (if $r_{h}=0$ and $v^{h}<x_{1}^{h}$ ). But this contradicts the fact that $\mathbf{v} \in \mathcal{V}$. Observe that:

$$
P^{\mathbf{x}}(\mathbf{v})=\sum_{h=1}^{k} r_{h} v_{h}-\sum_{h=1}^{k} \sum_{i \leq r_{h}} x_{i}^{h}
$$

Let $\left(r_{1}^{\prime}, \ldots, r_{k}^{\prime}\right) \in \Pi(\mathbf{y}, m)$ be any vector of ranks such that $\sum_{g=1}^{h} r_{g}^{\prime} \geq \sum_{g=1}^{h} r_{g}$ for any $h$ and $\sum_{h=1}^{k} \sum_{i \leq r_{h}} x_{i}^{h} \geq \sum_{h=1}^{k} \sum_{i \leq r_{h}^{\prime}} y_{i}^{h}$. Such a vector of ranks exists because $\mathbf{x} \succsim^{C L I} \mathbf{y}$. We then have:

$$
\begin{aligned}
P^{\mathbf{y}}(\mathbf{v}) & =\sum_{h=1}^{k} \sum_{i=1}^{n(h)} \max \left\{0, v_{h}-y_{i}^{h}\right\} \geq \sum_{h=1}^{k} \sum_{i \leq r_{h}^{\prime}} \max \left\{0, v_{h}-y_{i}^{h}\right\} \\
& \geq \sum_{h=1}^{k} \sum_{i \leq r_{h}^{\prime}}\left(v_{h}-y_{i}^{h}\right)=\sum_{h=1}^{k} r_{h}^{\prime} v_{h}-\sum_{h=1}^{k} \sum_{i \leq r_{h}^{\prime}} y_{i}^{h} .
\end{aligned}
$$

Note that

$$
\sum_{h=1}^{k} r_{h}^{\prime} v_{h}=\sum_{h=1}^{k} r_{h} v_{h}+\sum_{h=1}^{k-1}\left(v_{h}-v_{h+1}\right)\left(\sum_{g=1}^{h} r_{g}^{\prime}-\sum_{g=1}^{h} r_{g}\right) \geq \sum_{h=1}^{k} r_{h} v_{h}
$$

(because $\sum_{g=1}^{h} r_{g}^{\prime} \geq \sum_{g=1}^{h} r_{g}$ and $\left(v_{h}-v_{h+1}\right) \geq 0$ for any $\left.h\right)$. It follows that $P^{\mathbf{y}}(\mathbf{v}) \geq P^{\mathbf{x}}(\mathbf{v})$, as required.

Assume now that $\mathbf{x} \succsim O P G \mathbf{y}$. Take any integer $m \in\{1, \ldots, n\}$ and any $\left(r_{1}, \ldots, r_{k}\right) \in \Pi(\mathbf{x}, m)$. Define the set $F\left(\mathbf{y}, r_{1}, \ldots, r_{k}\right)$ by:

$$
F\left(\mathbf{y}, r_{1}, \ldots, r_{k}\right)=\left\{\left(i_{1}, \ldots, i_{k}\right) \in \Pi(\mathbf{y}, m): \sum_{g=1}^{h} i_{g} \geq \sum_{g=1}^{h} r_{g} \forall h, \sum_{g=1}^{k} i_{g}=m\right\}
$$

This set is nonempty. Indeed, if $m \in\left\{\sum_{g=1}^{h-1} n_{g}+1, \ldots, \sum_{g=1}^{h} n_{g}\right\}$ for some $h=1, \ldots, k$, then the combination of ranks $\left(i_{1}, \ldots, i_{k}\right):=\left(n_{1}, n_{2}, \ldots, n_{h-1}, m-\sum_{g=1}^{h-1} n_{g}, 0, \ldots, 0\right)$ belongs to $\Pi(\mathbf{y}, m)$ by construction. Moreover, we have

$$
\sum_{g=1}^{\widehat{h}} i_{g}=\sum_{g=1}^{\widehat{h}} n_{g} \geq \sum_{g=1}^{\widehat{h}} r_{g} \forall \widehat{h}=1, \ldots, h-1
$$

and

$$
\sum_{g=1}^{\widehat{h}} i_{g}=m \geq \sum_{g=1}^{\widehat{\hat{h}}} r_{g} \forall \widehat{\hat{h}}=h, \ldots, k
$$


Hence $\left(i_{1}, \ldots, i_{k}\right) \in F\left(\mathbf{y}, r_{1}, \ldots, r_{k}\right)$. The nonempty set $F\left(\mathbf{y}, r_{1}, \ldots, r_{k}\right)$ thus contains all admissible (as per set $\Pi$ ) lists of ranks for distribution $\mathbf{y}$ that satisfy Inequality (7) of Definition 5 vis-à-vis the list of ranks $\left(r_{1}, \ldots, r_{k}\right)$. Define now the vector of ranks $\left(r_{1}^{\prime}, \ldots, r_{k}^{\prime}\right) \in$ $\left\{0, \ldots, n_{1}\right\} \times \ldots \times\left\{0, \ldots, n_{k}\right\}$ by:

$$
\left(r_{1}^{\prime}, \ldots, r_{k}^{\prime}\right) \in \underset{\left(i_{1}, \ldots, i_{k}\right) \in F\left(\mathbf{y}, r_{1}, \ldots, r_{k}\right)}{\arg \min } \sum_{h=1}^{k} \sum_{i \leq i_{h}} y_{i}^{h} .
$$

Hence, the list of ranks $\left(r_{1}^{\prime}, \ldots, r_{k}^{\prime}\right)$ are those that define a set of $m$ "poorest" agents in situation $\mathbf{y}$, under the constraint that $\sum_{g=1}^{h} r_{g}^{\prime} \geq \sum_{g=1}^{h} r_{g}$ for any $h$. Consider now the vector of poverty lines $\mathbf{v}$ defined as follows (for any $h=1, \ldots, k)^{5}$ :

$$
v_{h}:=\max \left\{\left\{y_{r_{l}}^{l}: l \geq h\right\} \cup\left\{y_{r_{g}}^{g}: g<h \text { and } \sum_{e=1}^{j} r_{e}^{\prime}>\sum_{e=1}^{j} r_{e} \forall j=g, \ldots, h-1\right\}\right\} .
$$

This vector $\mathbf{v}$ is well defined because, for every $h$, at least one of sets $\left\{y_{r_{l}}^{l}: l \geq h\right\}$ or $\left\{y_{r_{g}}^{g}\right.$ : $g<h$ and $\left.\sum_{e=1}^{j} r_{e}^{\prime}>\sum_{e=1}^{j} r_{e} \forall j=g, \ldots, h-1\right\}$ is not empty. Let us first check that $\mathbf{v} \in \mathcal{V}$. This amounts to showing that if $h$ and $h^{\prime}$ are two categories such that $h<h^{\prime}$, then $v_{h} \geq$ $v_{h^{\prime}}$. But this is an immediate consequence of the fact that, for any $h$ and $h^{\prime}$ such that $h<$ $h^{\prime}$, set $\left\{y_{r_{l}}^{l}: l \geq h\right\} \cup\left\{y_{r_{l}}^{g}: g<h\right.$ and $\left.\sum_{e=1}^{j} r_{e}^{\prime}>\sum_{e=1}^{j} r_{e} \forall j=g, \ldots, h-1\right\}$ contains set $\left\{y_{r_{l}}^{l}: l \geq\right.$ $\left.h^{\prime}\right\} \cup\left\{y_{r_{l}}^{g}: g<h^{\prime}\right.$ and $\left.\sum_{e=1}^{j} r_{e}^{\prime}>\sum_{e=1}^{j} r_{e} \forall j=g, \ldots, h^{\prime}-1\right\}$ as a subset. As a result, the maximum taken over the larger set cannot be smaller than the maximum taken over the subset. We now prove that, for any $h=1, \ldots, k$, we have $v_{h} \in\left[y_{r_{h}}^{h}, y_{r_{h}+1}^{h}\right]$. By definition of $v_{h}$, the only inequality that needs to be established is $v_{h} \leq y_{r_{h}+1}^{h}$. By contradiction, assume that $v_{h}>y_{r_{h}+1}^{h}$. Since $\left(r_{1}^{\prime}, \ldots, r_{k}^{\prime}\right) \in \Pi(\mathbf{y}, m)$, we have $y_{r_{j}^{\prime}}^{j}<y_{r_{g}^{\prime}+1}^{g}$ for every two categories $g$ and $j$ such that $g<j$. Hence the fact that $v_{h}>y_{r_{h}+1}^{h}$ may only be due to the existence of a category $g<h$ such that $\sum_{e=1}^{l} r_{e}^{\prime}>\sum_{e=1}^{l} r_{e} \forall l=g, \ldots, h-1$ and $y_{r_{g}^{\prime}}^{g}>y_{r_{h}^{\prime}+1}^{h}$. Consider then the list of ranks $\left(r_{1}^{\prime \prime}, \ldots, r_{k}^{\prime \prime}\right) \in\left\{0, \ldots, n_{1}\right\} \times \ldots \times\left\{0, \ldots, n_{k}\right\}$ defined by $r_{e}^{\prime \prime}=r_{e}^{\prime}$ for $e \neq g, h, r_{h}^{\prime \prime}=r_{h}^{\prime}+1$ and $r_{g}^{\prime \prime}=r_{g}^{\prime}-1$ Observe that $\left(r_{1}^{\prime \prime}, \ldots, r_{k}^{\prime \prime}\right) \in F\left(y, r_{1}, \ldots, r_{k}\right)$. Now:

$$
\sum_{h=1}^{k} \sum_{i \leq r_{h}^{\prime \prime}} y_{i}^{h}=\sum_{h=1}^{k} \sum_{i \leq r_{h}^{\prime}} y_{i}^{h}+y_{r_{h}^{\prime}+1}^{h}-y_{r_{g}^{\prime}}^{g}<\sum_{h=1}^{k} \sum_{i \leq r_{h}^{\prime}} y_{i}^{h}
$$

(because $\left.y_{r_{g}^{\prime}}^{g}>y_{r_{h}^{\prime}+1}^{h}\right)$. But this contradicts the definition of $\left(r_{1}^{\prime}, \ldots, r_{k}^{\prime}\right)$ provided by (A.2).

Since $y_{r_{h}^{\prime}+1}^{h} \geq v_{h} \geq y_{r_{h}^{\prime}}^{h}$ for all $h$, we have:

$$
P^{\mathbf{y}}(\mathbf{v})=\sum_{h=1}^{k} \sum_{i \leq r_{h}^{\prime}}\left(v_{h}-y_{i}^{h}\right)=\sum_{h=1}^{k} r_{h}^{\prime} v_{h}-\sum_{h=1}^{k} \sum_{i \leq r_{h}^{\prime}} y_{i}^{h} .
$$

\footnotetext{
${ }^{5}$ Again with the convention that, if $r_{h}^{\prime}=0$, then $y_{r_{h}^{\prime}}^{h}=\underline{v}(\mathbf{x}, \mathbf{y})$.
} 
and:

$$
\begin{aligned}
P^{\mathbf{x}}(\mathbf{v}) & =\sum_{h=1}^{k} \sum_{i=1}^{n(h)} \max \left\{0, v_{h}-x_{i}^{h}\right\} \geq \sum_{h=1}^{k} \sum_{i \leq r_{h}} \max \left\{0, v_{h}-x_{i}^{h}\right\} \\
& \geq \sum_{h=1}^{k} \sum_{i \leq r_{h}}\left(v_{h}-x_{i}^{h}\right)=\sum_{h=1}^{k} r_{h} v_{h}-\sum_{h=1}^{k} \sum_{i \leq i_{h}} x_{i}^{h} .
\end{aligned}
$$

Now, as established above:

$$
\sum_{h=1}^{k} r_{h}^{\prime} v_{h}=\sum_{h=1}^{k} r_{h} v_{h}+\sum_{h=1}^{k-1}\left(v_{h}-v_{h+1}\right)\left(\sum_{g=1}^{h} r_{g}^{\prime}-\sum_{g=1}^{h} r_{g}\right) .
$$

Let $h \leq k-1$. By construction of the vector of poverty line $\mathbf{v}$, if $\sum_{g=1}^{h} r_{g}^{\prime}>\sum_{g=1}^{h} r_{g}$ then $v_{h}=v_{h+1}$. Indeed let $g_{0}$ be the category such that $\sum_{e=1}^{g} r_{e}^{\prime}>\sum_{e=1}^{g} r_{e}$ for $g=g_{0}, \ldots, h$ and $\sum_{e=1}^{g_{0}-1} r_{e}^{\prime}=\sum_{e=1}^{g_{0}-1} r_{e}$. Then $v_{h}=v_{h+1}=\max _{g \geq g_{0}} y_{i_{g}}^{g}$. It follows that $\sum_{h=1}^{k} r_{h}^{\prime} v_{h}=\sum_{h=1}^{k} r_{h} v_{h}$.

By assumption, $P^{\mathbf{y}}(\mathbf{v}) \geq P^{\mathbf{x}}(\mathbf{v})$. Hence

$$
\sum_{h=1}^{k} \sum_{i \leq r_{h}^{\prime}} y_{i}^{h}=\sum_{h=1}^{k} r_{h}^{\prime} v_{h}-P^{\mathbf{y}}(\mathbf{v}) \leq \sum_{h=1}^{k} r_{h} v_{h}-P^{\mathbf{x}}(\mathbf{v}) \leq \sum_{h=1}^{k} \sum_{i \leq r_{h}} x_{i}^{h}
$$

which proves the result.

A.4 Some Technical Lemmas. In this section, we prove some technical lemmas. Note that we assume without loss of generality that, in the two distributions $\mathbf{x}$ and $\mathbf{y}$ under consideration, we have $x_{i}^{h} \neq y_{j}^{h}$ for every type $h=1, \ldots, k$ and every $i, j \in \mathcal{N}(h)$. In effect, if this condition was not satisfied, that is, if there was a type $h$ for which $x_{i}^{h}=y_{j}^{h}$ for some $i, j \in \mathcal{N}(h)$, these two agents could be removed and we could proceed with the remaining population. Since the OPG criterion is additively separable, such a removal of agents with the same type and income from distributions $\mathbf{x}$ and $\mathbf{y}$ would not affect their ranking as per the OPG criterion.

The first auxiliary result of this section is the following lemma (proved, like all lemmas and formal claims in the Appendix), which says that if $\mathbf{x}$ is a distribution that dominates $\mathbf{y}$ for the OPG criterion, the poorest person in the worst category is weakly richer in $\mathbf{x}$ than in $\mathbf{y}$ and, conversely, the richest person in the best category is poorer in $\mathbf{x}$ than in $\mathbf{y}$.

Lemma A.1. Let $\mathbf{x}$ and $\mathbf{y}$ be distributions in $\mathcal{D}(I)$, for which $\mathbf{x} \succsim^{O P G} \mathbf{y}$. Then $y_{1}^{1}<x_{1}^{1}$ and $y_{n(k)}^{k}>x_{n(k)}^{k}$.

Proof. For the first statement, assume by contraposition that $y_{1}^{1}>x_{1}^{1}$. Consider then the vector of poverty lines $\left(y_{1}^{1}, \underline{v}(\mathbf{x}, \mathbf{y}), \ldots, \underline{v}(\mathbf{x}, \mathbf{y})\right) \in \mathcal{V}$. One has:

$$
\begin{aligned}
& P^{\mathbf{y}}\left(y_{1}^{1}, \underline{v}(\mathbf{x}, \mathbf{y}), \ldots, \underline{v}(\mathbf{x}, \mathbf{y})\right)=0 \text { and: } \\
& P^{\mathbf{x}}\left(y_{1}^{1}, \underline{v}(\mathbf{x}, \mathbf{y}), \ldots, \underline{v}(\mathbf{x}, \mathbf{y})\right) \geq y_{1}^{1}-x_{1}^{1}>0
\end{aligned}
$$

so that $x \succsim^{O P G} y$ does not hold, as required. The second statement holds by a mirror argument. 
The next lemma states that, if a distribution $\mathbf{x}$ dominates a distribution $\mathbf{y}$ by the OPG criterion, then the sum of incomes held by agents in the $\bar{h}$ lowest categories must be larger or equal in $\mathbf{x}$ than in $\mathbf{y}$ for any $\bar{h}$.

Lemma A.2. Let $\mathbf{x}$ and $\mathbf{y}$ be two distributions in $\mathcal{D}(I)$ for which $\mathbf{x} \succsim^{O P G} \mathbf{y}$. Then $\sum_{h=1}^{\bar{h}} \sum_{i=1}^{n(h)} x_{i}^{h} \geq \sum_{h=1}^{\bar{h}} \sum_{i=1}^{n(h)} y_{i}^{h}$ for all $\bar{h}=1, \ldots, k$.

Proof. For any type $\bar{h}=1, \ldots, k$, the vector of poverty lines $v^{\bar{h}} \in D^{k}$ defined by:

$$
\mathbf{v}^{\bar{h}}=(\underbrace{\bar{v}(\mathbf{x}, \mathbf{y}), \ldots, \bar{v}(\mathbf{x}, \mathbf{y})}_{\bar{h} \quad k-\bar{h}}, \underbrace{v(\mathbf{x}, \mathbf{y}), \ldots, \underline{v}(\mathbf{x}, \mathbf{y})})
$$

clearly belongs to $\mathcal{V}$. Hence, since $x \succsim^{O P G} y$, we have:

$$
\begin{aligned}
P^{\mathbf{x}}\left(\mathbf{v}^{\bar{h}}\right) & \leq P^{\mathbf{y}}\left(\mathbf{v}^{\bar{h}}\right) \\
& \Longleftrightarrow \\
\sum_{h=1}^{\bar{h}} n(h) \bar{v}(\mathbf{x}, \mathbf{y})-\sum_{h=1}^{\bar{h}} \sum_{i=1}^{n(h)} x_{i}^{h} & \leq \sum_{h=1}^{\bar{h}} n(h) \bar{v}(\mathbf{x}, \mathbf{y})-\sum_{h=1}^{\bar{h}} \sum_{i=1}^{n(h)} y_{i}^{h} \\
& \Longleftrightarrow \\
\sum_{h=1}^{\bar{h}} \sum_{i=1}^{n(h)} x_{i}^{h} & \geq \sum_{h=1}^{\bar{h}} \sum_{i=1}^{n(h)} y_{i}^{h} .
\end{aligned}
$$

We next state an important lemma that provides a sufficient condition for performing an FIP from distribution $\mathbf{y}$ in such a way that the distribution obtained after making such an FIP remains dominated by $\mathbf{x}$ as per the OPG criterion. For any income poverty threshold $t \in \mathbb{R}$ and any distribution $\mathbf{x}$, we also denote by $\overline{\mathcal{P}}^{\mathbf{x}}(h, t)$ and $\mathcal{P}^{\mathbf{x}}(h, t)$ the (possibly empty) sets of agents of type $h$ who are, respectively, weakly and strictly poor for threshold $t$ in distribution $\mathbf{x}$. These sets are defined by:

$$
\overline{\mathcal{P}}^{\mathbf{x}}(h, t)=\left\{i \in \mathcal{N}(h): x_{i}^{h} \leq t\right\} \text { and } \mathcal{P}^{\mathbf{x}}(h, t)=\left\{i \in \mathcal{N}(h): x_{i}^{h}<t\right\}
$$

whereas the number of poor that these sets contain are denoted, respectively, by $\bar{p}^{\mathbf{x}}(h, t)=$ $\# \overline{\mathcal{P}}^{\mathbf{x}}(h, t)$ and $p^{\mathbf{x}}(h, t)=\# \mathcal{P}^{\mathbf{x}}(h, t)$.

Lemma A.3. Let $\mathbf{x}$ and $\mathbf{y}$ be two distributions in $\mathcal{D}(I)$ such that $\mathbf{x} \succsim^{O P G} \mathbf{y}$. Assume that

- there exist $\mathbf{w} \in \mathcal{V}, i_{1} \in \mathcal{N}(1)$ and $h_{0} \in\{2, \ldots, k\}$ such that ${ }^{6}$

$$
P^{\mathbf{y}}(\mathbf{w})=P^{\mathbf{x}}(\mathbf{w}), y_{i_{1}}^{1}=w_{1}=\cdots=w_{h_{0}}>w_{h_{0}+1} ;
$$

- there exists a category $g_{0}$ such that: $2 \leq g_{0} \leq h_{0}$ and:

$$
\sum_{h=l+1}^{g_{0}} \bar{p}^{\mathbf{y}}\left(h, y_{i_{1}}^{1}\right)<\sum_{h=l+1}^{g_{0}} \bar{p}^{\mathbf{x}}\left(h, y_{i_{1}}^{1}\right) \forall l=1, \ldots, g_{0}-1 .
$$

Then there exists a distribution $\overline{\mathbf{x}} \in \mathcal{D}(I)$ such that $\overline{\mathbf{x}}$ is obtained from $\mathbf{y}$ by an FIP and $\mathbf{x} \succsim^{O P G}$ $\overline{\mathbf{X}}$.

\footnotetext{
${ }^{6}$ With the convention that $w_{k+1}=\underline{v}(\mathbf{x}, \mathbf{y})$
} 
Proof. By inequality (A.4) there exists at least one agent with income strictly larger than $y_{i_{1}}^{1}$ in one of the categories $\left\{2, \ldots, g_{0}\right\}$. Hence we can define $y^{*}:=$ $\min \left\{y_{i}^{g}: 2 \leq g \leq g_{0}, i \in \mathcal{N}(g), y_{i}^{g}>y_{i_{1}}^{1}\right\}$. We also define $\gamma \in\left\{2, \ldots, g_{0}\right\}$ and $i_{\gamma} \in \mathcal{N}(\gamma)$ by

$$
\gamma:=\min \left\{g \geq 2: \exists i \in \mathcal{N}(g) \text { such that } y_{i}^{g}=y^{*}\right\}, i_{\gamma}:=\min \left\{i \in \mathcal{N}(\gamma): y_{i}^{\gamma}=y^{*}\right\}
$$

Namely, $y_{i_{\gamma}}^{\gamma}$ corresponds to the smallest income in distribution $\mathbf{y}$, among the incomes that are strictly larger than $y_{i_{1}}^{1}$. We now prove that for any $\mathbf{v} \in \mathcal{V}$, we have:

$$
P^{\mathbf{y}}(\mathbf{v})-P^{\mathbf{x}}(\mathbf{v}) \geq \min \left\{y_{i_{\gamma}}^{\gamma}, v_{1}\right\}-\max \left\{y_{i_{1}}^{1}, v_{\gamma}\right\}
$$

If either $v_{1} \leq y_{i_{1}}^{1}$ or $v_{\gamma} \geq y_{i_{\gamma}}^{\gamma}$, inequality (A.5) trivially holds (because $\min \left\{y_{i_{\gamma}}^{\gamma}, v_{1}\right\}-$ $\max \left\{y_{i_{1}}^{1}, v_{\gamma}\right\} \leq 0$ in this case). Hence we suppose that $v_{1}>y_{i_{1}}^{1}$ and $v_{\gamma}<y_{i_{\gamma}}^{\gamma}$. We establish the result by considering three different cases.

Case (i): $y_{i_{1}}^{1} \leq v_{g_{0}} \leq v_{1} \leq y_{i_{\gamma}}^{\gamma}$.

By definition of $y_{i_{\gamma}}^{\gamma}$, we have that that:

$$
\bar{p}^{\mathrm{y}}(h, w)=\bar{p}^{\mathrm{y}}\left(h, w^{\prime}\right)
$$

for $h=2, \ldots, g_{0}$ and any $w$ and $w^{\prime} \in\left[y_{i_{1}}^{1}, y_{i_{\gamma}}^{\gamma}\right]$. Indeed, the number of poor in categories $2, . ., g_{0}$ at distribution $\mathbf{y}$ does not change when we move the poverty line applicable to all these categories from $y_{i_{1}}^{1}$ to $y_{i_{\gamma}}^{\gamma}$ ). Combining this with inequality (A.4) we obtain that

$$
\sum_{g=h}^{g_{0}}\left[\bar{p}^{\mathbf{y}}\left(g, v_{g}\right)-\bar{p}^{\mathbf{x}}\left(g, v_{g}\right)\right] \leq-1, \quad \forall h=2, \ldots, g_{0} .
$$

Thus, we have:

$$
\begin{aligned}
& \sum_{g=2}^{g_{0}} \sum_{i=1}^{n(g)}\left[\max \left(v_{1}-y_{i}^{g}, 0\right)-\max \left(v_{1}-x_{i}^{g}, 0\right)\right] \\
\leq & \sum_{g=2}^{g_{0}} \sum_{i=1}^{n(g)}\left[\max \left(v_{g}-y_{i}^{g}, 0\right)-\max \left(v_{g}-x_{i}^{g}, 0\right)\right] \\
+ & \sum_{g=2}^{g_{0}}\left[\bar{p}^{\mathbf{y}}\left(g, v_{g}\right)-\bar{p}^{\mathbf{x}}\left(g, v_{g}\right)\right]\left(v_{1}-v_{g}\right) \\
= & \sum_{g=2}^{g_{0}} \sum_{i=1}^{n(g)}\left[\max \left(v_{g}-y_{i}^{g}, 0\right)-\max \left(v_{g}-x_{i}^{g}, 0\right)\right] \\
+ & \sum_{h=2}^{g_{0}} \sum_{g=h}^{g_{0}}\left[\bar{p}^{\mathbf{y}}\left(g, v_{g}\right)-\bar{p}^{\mathbf{x}}\left(g, v_{g}\right)\right]\left(v_{h-1}-v_{h}\right) \\
\leq & \sum_{g=2}^{g_{0}} \sum_{i=1}^{n(g)}\left[\max \left(v_{g}-y_{i}^{g}, 0\right)-\max \left(v_{g}-x_{i}^{g}, 0\right)\right]+v_{g_{0}}-v_{1} .
\end{aligned}
$$


Hence:

$$
\begin{aligned}
P^{\mathbf{y}}(\mathbf{v})-P^{\mathbf{x}}(\mathbf{v})= & P^{\mathbf{y}}\left(v_{1}, \ldots, v_{1}, v_{g_{0}+1}, \ldots, v_{k}\right)-P^{\mathbf{x}}\left(v_{1}, \ldots, v_{1}, v_{g_{0}+1}, \ldots, v_{k}\right) \\
& -\sum_{g=2}^{g_{0}} \sum_{i=1}^{n(g)}\left[\max \left(v_{1}-y_{i}^{g}, 0\right)-\max \left(v_{1}-x_{i}^{g}, 0\right)\right] \\
& +\sum_{g=2}^{g_{0}} \sum_{i=1}^{n(g)}\left[\max \left(v_{g}-y_{i}^{g}, 0\right)-\max \left(v_{g}-x_{i}^{g}, 0\right)\right] \\
\geq & P^{\mathbf{y}}\left(v_{1}, \ldots, v_{1}, v_{g_{0}+1}, \ldots, v_{k}\right) \\
& -P^{\mathbf{x}}\left(v_{1}, \ldots, v_{1}, v_{g_{0}+1}, \ldots, v_{k}\right)+v_{1}-v_{g_{0}} \\
\geq & v_{1}-v_{g_{0}}\left(\operatorname{because}\left(v_{1}, \ldots, v_{1}, v_{s_{0}+1}, \ldots, v_{k}\right) \in \mathcal{V}\right) \\
\geq & v_{1}-v_{\gamma} \\
= & \min \left\{y_{i_{\gamma}}^{\gamma}, v_{1}\right\}-\max \left\{y_{i_{1}}^{1}, v_{\gamma}\right\}
\end{aligned}
$$

as required.

Case (ii): $v_{1}>y_{i_{\gamma}}^{\gamma}$ and $v_{g_{0}} \geq y_{i_{1}}^{1}$. In this case, there exists some $\underline{h} \in\{1, \ldots, \gamma-1\}$ such that $v_{1} \geq \ldots \geq v_{\underline{h}}>y_{i_{\gamma}}^{\gamma} \geq v_{\underline{h}+1} \geq \ldots \geq v_{k}$. Let $\tilde{v}=\left(y_{i_{\gamma}}^{\gamma}, \ldots, y_{i_{\gamma}}^{\gamma}, v_{\underline{h}+1}, \ldots, v_{k}\right)$. Then $\tilde{v}$ belongs to case $(i)$ and, consequently:

$$
P^{\mathbf{y}}(\tilde{\mathbf{v}})-P^{\mathbf{x}}(\tilde{\mathbf{v}}) \geq \tilde{v}_{1}-\tilde{v}_{\gamma}=y_{i_{1}}^{1}-v_{\gamma} .
$$

Moreover denoting $\hat{v}:=\left(v_{1}, \ldots, v_{\underline{h}}, v_{\underline{h}+1}^{c}, \ldots, v_{k}^{c}\right)$, we have, by definition of $v^{c}$ :

$$
\begin{aligned}
\left(P^{\mathbf{y}}(\tilde{\mathbf{v}})-P^{\mathbf{x}}(\tilde{\mathbf{v}})\right)-\left(P^{\mathbf{y}}(\mathbf{v})-P^{\mathbf{x}}(\mathbf{v})\right) & =\left(P^{\mathbf{y}}\left(\mathbf{v}^{c}\right)-P^{\mathbf{x}}\left(\mathbf{v}^{c}\right)\right)-\left(P^{\mathbf{y}}(\hat{\mathbf{v}})-P^{\mathbf{x}}(\hat{\mathbf{v}})\right) \\
& =P^{\mathbf{x}}(\hat{\mathbf{v}})-P^{\mathbf{x}}(\hat{\mathbf{v}}) \\
& \leq 0 .
\end{aligned}
$$

Hence we have:

$$
P^{\mathbf{y}}(\mathbf{v})-P^{\mathbf{x}}(\mathbf{v}) \geq P^{\mathbf{y}}(\tilde{\mathbf{v}})-P^{\mathbf{x}}(\tilde{\mathbf{v}}) \geq y_{i_{\gamma}}^{\gamma}-v_{\gamma}=\min \left\{y_{i_{\gamma}}^{\gamma}, v_{1}\right\}-\max \left\{y_{i_{1}}^{1}, v_{\gamma}\right\}
$$

as required.

Case (iii): $v_{g_{0}}<y_{i_{1}}$. (without any assumption on the relative standing of $v_{1}$ vis-à-vis $y_{i_{\gamma}}^{\gamma}$ ) In this case, there exists some $\bar{h}<\left\{1, \ldots, g_{0}-1\right\}$ such that $v_{\bar{h}} \geq y_{i_{1}}^{1}>v_{\bar{h}+1} \geq \ldots \geq v_{k}$. We first note that:

$$
\begin{aligned}
& \sum_{g=\bar{h}+1}^{k} \sum_{i=1}^{n(g)}\left[\max \left(v_{g}-y_{i}^{g}, 0\right)-\max \left(v_{1}-x_{i}^{g}, 0\right)\right] \\
\geq & \sum_{g=\bar{h}+1}^{k} \sum_{i=1}^{n(g)}\left[\max \left(w_{g}-y_{i}^{g}, 0\right)-\max \left(w_{1}-x_{i}^{g}, 0\right)\right]
\end{aligned}
$$


because assuming otherwise would imply that:

$$
\begin{aligned}
P^{\mathbf{y}}\left(w_{1}, \ldots, w_{\bar{h}}, v_{\bar{h}+1}, \ldots, v_{k}\right)-P^{\mathbf{x}}\left(w_{1}, \ldots, w_{\bar{h}}, v_{\bar{h}+1}, \ldots, v_{k}\right) & <P^{\mathbf{y}}(\mathbf{w})-P^{\mathbf{x}}(\mathbf{w}) \\
& =0
\end{aligned}
$$

and this inequality contradicts the statement that $x \succsim^{O P G} y$ (since the vector of poverty lines $\left(w_{1}, \ldots, w_{\bar{h}}, v_{\bar{h}+1}, \ldots, v_{k}\right)$ belongs to $\left.V\right)$.

Let $\tilde{v}:=\left(v_{1}, \ldots, v_{\bar{h}}, w_{\bar{h}+1}, \ldots, w_{k}\right) \in \mathcal{V}$. Observe with care that the vector $\tilde{v}$ so defined corresponds either to case (i) (if $v_{1} \leq y_{i_{\gamma}}^{\gamma}$ ) or to case (ii) (if $v_{1}>y_{i_{\gamma}}^{\gamma}$ ). Observe also that $\max \left\{y_{i_{1}}^{1}, \tilde{v}_{\gamma}\right\} \leq \max \left\{y_{i_{1}}^{1}, v_{\gamma}\right\}$. Indeed if $\tilde{v}_{\gamma} \leq y_{i_{1}}^{1}$ there is nothing to prove. If on the other hand $\tilde{v}_{\gamma}>y_{i_{1}}^{1}$, then $\tilde{v}_{\gamma}=v_{\gamma}$ by definition of $\bar{h}$ and the inequality $\max \left\{y_{i_{1}}^{1}, \tilde{v}_{\gamma}\right\} \leq \max \left\{y_{i_{1}}^{1}, v_{\gamma}\right\}$ also holds. Collecting these observations, we obtain that

$$
\begin{aligned}
P^{\mathbf{y}}(\mathbf{v})-P^{\mathbf{x}}(\mathbf{v}) & \geq P^{\mathbf{y}}(\widetilde{\mathbf{v}})-P^{\mathbf{x}}(\widetilde{\mathbf{v}}) \\
& \geq \min \left\{y_{i_{\gamma}}^{\gamma}, v_{1}\right\}-\max \left\{\tilde{v}_{\gamma}, y_{i_{1}}^{1}\right\} \text { (by cases (i) or (ii)) } \\
& \geq \min \left\{y_{i_{\gamma}}^{\gamma}, v_{1}\right\}-\max \left\{y_{i_{1}}^{1}, v_{\gamma}\right\},
\end{aligned}
$$

which proves (A.5) in that last case.

Let us now establish the existence of a distribution $\overline{\mathbf{x}} \in D(I)$ that is obtained from $\mathbf{y}$ by an FIP and that is such that $\mathbf{x} \succsim^{O P G} \overline{\mathbf{x}}$. Let $\overline{\mathbf{x}}$ be the distribution obtained from $\mathbf{y}$ by means of an FIP from agent $i^{\gamma} \in N(\gamma)$ to agent $i^{1} \in N(1)$. Let us show that $\mathbf{x} \succsim^{O P G} \overline{\mathbf{x}}$. Consider any vector $v \in \mathcal{V}$ of ordered poverty lines. If $v_{\gamma} \geq y_{i^{\gamma}}^{\gamma}$ or $v_{1} \leq y_{i^{1}}^{1}$, it is clear that $P^{\overline{\mathbf{x}}}(\mathbf{v})=P^{\mathbf{y}}(\mathbf{v}) \geq P^{\mathbf{x}}(\mathbf{v})$. If on the other hand $v_{\gamma}<y_{i^{\gamma}}^{\gamma}$ and $v_{1}>y_{i^{1}}^{1}$, by straightforward computations ${ }^{7}$ :

$$
\begin{aligned}
P^{\overline{\mathbf{x}}}(\mathbf{v})= & P^{\mathbf{y}}(\mathbf{v})-\max \left\{y_{i^{\gamma}}^{\gamma}-v_{\gamma}, 0\right\}-\max \left\{y_{i^{1}}^{1}-v_{1}, 0\right\} \\
& +\max \left\{y_{i^{1}}^{1}-v_{\gamma}, 0\right\}+\max \left\{y_{i^{\gamma}}^{\gamma}-v_{1}, 0\right\} \\
= & P^{\mathbf{y}}(\mathbf{v})-y_{i^{\gamma}}^{\gamma}+v_{\gamma}+\max \left\{y_{i^{1}}^{1}-v_{\gamma}, 0\right\}+\max \left\{y_{i^{\gamma}}^{\gamma}-v_{1}, 0\right\} \\
= & P^{\mathbf{y}}(\mathbf{v})-\left(y_{i^{\gamma}}^{\gamma}-\max \left\{y_{i^{\gamma}}^{\gamma}-v_{1}, 0\right\}\right)+\left(v_{\gamma}-\max \left\{y_{i^{1}}^{1}-v_{\gamma}, 0\right\}\right) \\
= & P^{\mathbf{y}}(\mathbf{v})-\min \left\{y_{i^{\gamma}}^{\gamma}, v_{1}\right\}+\max \left\{y_{i^{1}}^{1}, v_{\gamma}\right\} .
\end{aligned}
$$

Using the inequality (A.5) proved above, this implies that:

$$
P^{\overline{\mathbf{x}}}(\mathbf{v})-P^{\mathbf{x}}(\mathbf{v}) \geq P^{\mathbf{y}}(\mathbf{v})-P^{\mathbf{x}}(\mathbf{v})-\min \left\{y_{i^{\gamma}}^{\gamma}, v_{1}\right\}+\max \left\{y_{i^{\gamma}}^{1}, v_{\gamma}\right\} \geq 0,
$$

which proves the result.

Although this result is important, it is of limited immediate usefulness. There are actually no obvious ways to identify the poverty lines vector $\mathbf{w}$ that is required by this lemma. We will nonetheless use Lemma A.3 on two occasions in what follows.

\section{A.5 Case (A): Proof of Propositions 1 and 2}

We start by establishing the following important result: if an ordered list $\mathbf{v} \in \mathcal{V}$ of poverty lines is such that $v_{1}>y_{1}^{1}$ and $v_{h_{0}}<v_{h_{0}}^{c}$ for some $h_{0} \in\{2, \ldots, k\}$, then $P^{\mathbf{x}}(\mathbf{v})<P^{\mathbf{y}}(\mathbf{v})$. Specifically, we prove the following result. 
Lemma A.4. Let $\mathbf{x}$ and $\mathbf{y}$ be two distributions in $\mathcal{D}(I)$ such that $\mathbf{x} \succsim^{O P G} \mathbf{y}$. Suppose that $v_{1}^{c}>y_{1}^{1}$. Then, if $\mathbf{v} \in \mathcal{V}$ is such that $v_{1}>y_{1}^{1}$ and $v_{h_{0}}<v_{h_{0}}^{c}$ for some $h_{0} \in\{2, \ldots, k\}$, we will have $P^{\mathbf{x}}(\mathbf{v})<P^{\mathbf{y}}(\mathbf{v})$.

Proof. Define the two vectors of poverty lines $v^{-}$and $v^{+}$by:

$$
\begin{aligned}
& v_{h}^{-}=\min \left(v_{h}, v_{h}^{c}\right) \text { and }, \\
& v_{h}^{+}=\max \left(v_{h}, v_{h}^{c}\right) .
\end{aligned}
$$

It is clear that $v^{-}$and $v^{+}$both belong to $\mathcal{V}$. By definition of $v^{-}$and $v^{+}$, we have:

$$
\begin{aligned}
& P^{\mathbf{x}}\left(\mathbf{v}^{+}\right)-P^{\mathbf{y}}\left(\mathbf{v}^{+}\right)+P^{\mathbf{x}}\left(\mathbf{v}^{-}\right)-P^{\mathbf{y}}\left(\mathbf{v}^{-}\right) \\
= & P^{\mathbf{x}}\left(\mathbf{v}^{c}\right)-P^{\mathbf{y}}\left(\mathbf{v}^{c}\right)+P^{\mathbf{x}}(\mathbf{v})-P^{\mathbf{y}}(\mathbf{v})
\end{aligned}
$$

By definition of $v^{c}$, we have $P^{\mathbf{x}}\left(v^{c}\right)-P^{\mathbf{y}}\left(v^{c}\right)=0$. Assume therefore by contradiction that $P^{\mathbf{x}}(\mathbf{v})-P^{\mathbf{y}}(\mathbf{v})=0$ so that, using equality (A.6), we have:

$$
P^{\mathbf{x}}\left(\mathbf{v}^{+}\right)-P^{\mathbf{y}}\left(\mathbf{v}^{+}\right)+P^{\mathbf{x}}\left(\mathbf{v}^{-}\right)-P^{\mathbf{y}}\left(\mathbf{v}^{-}\right)=0
$$

As there exists $h_{0}$ such that $v_{h_{0}}<v_{h_{0}}^{c}$, we must have $v_{h_{0}}^{-}=v_{h_{0}}<v_{h_{0}}^{c}$. Moreover $v_{1}^{-}=$ $\min \left(v_{1}, v_{1}^{c}\right)>y_{1}^{1}$. Consequently, by the recursive definition of $v^{c}$, we must have that:

$$
P^{\mathbf{x}}\left(\mathbf{v}^{-}\right)-P^{\mathbf{y}}\left(\mathbf{v}^{-}\right)<0 .
$$

But this implies that:

$$
P^{\mathbf{x}}\left(\mathbf{v}^{+}\right)-P^{\mathbf{y}}\left(\mathbf{v}^{+}\right)>0
$$

a contradiction of the fact that $x \succsim^{O P G} y$ and that $v^{+}$belongs to $\mathcal{V}$.

We now state as a corollary of Lemma A.4 the following alternative definition of the critical vector $\mathbf{v}^{c}$.

Corollary A.1. Let $\mathbf{x}$ and $\mathbf{y}$ be two distributions in $\mathcal{D}(I)$ such that $\mathbf{x} \succsim^{O P G} \mathbf{y}$. Suppose that $v_{1}^{c}>y_{1}^{1}$. Then, for every $h=2, \ldots, k$, we have:

$$
v_{h}^{c}=\min _{v_{h}}\left\{\exists v_{-h} \in[\underline{v}(\mathbf{x}, \mathbf{y}), \bar{v}(\mathbf{x}, \mathbf{y})]^{k-1}: v_{1}>y_{1}^{1}, \mathbf{v}=\left(v_{h}, v_{-h}\right) \in \mathcal{V} \text { and } P^{\mathbf{x}}(\mathbf{v})=P^{\mathbf{y}}(\mathbf{v})\right\}
$$

Proof. Using the recursive definition of the vector $v^{c}$ provided by (4), it is clear that:

$$
v_{h}^{c} \geq \min _{v_{h}}\left\{\exists v_{-h}: v_{1}>y_{1}^{1}, \mathbf{v}=\left(v_{h}, v_{-h}\right) \in \mathcal{V} \text { and } P^{\mathbf{x}}(\mathbf{v})=P^{\mathbf{y}}(\mathbf{v})\right\}
$$

for all $h$. In order to prove that:

$$
v_{h}^{c} \leq \min _{v_{h}}\left\{\exists v_{-h}: v_{1}>y_{1}^{1}, \mathbf{v}=\left(v_{h}, v_{-h}\right) \in \mathcal{V} \text { and } P^{\mathbf{x}}(\mathbf{v})=P^{\mathbf{y}}(\mathbf{v})\right\}
$$

we simply note that, thanks to Lemma A.4, any vector $v \in \mathcal{V}$ such that $v_{1}>y_{1}^{1}$ and $P^{\mathbf{x}}(\mathbf{v})-$ $P^{\mathbf{y}}(\mathbf{v})=0$ must also satisfy $v_{h} \geq v_{h}^{c}$ for all $h \in\{2, \ldots, k\}$. 
The next lemma establishes an important comparative statement about adjacent sets of strictly and weakly poor agents in $\mathbf{x}$ and $\mathbf{y}$ when these sets are defined with respect to the vector of ordered poverty lines $\mathbf{v}^{c}$ where the poverty lines assigned to the adjacent categories are the same. Specifically, the next lemma establishes the following:

Lemma A.5. Let $\mathbf{x}$ and $\mathbf{y}$ be two distributions in $\mathcal{D}(I)$ such that $\mathbf{x} \succsim^{O P G} \mathbf{y}$. Suppose that $v_{1}^{c}>y_{1}^{1}$. Then, for any $h_{0} \in\{1, \ldots, k\}$ and $\bar{h} \in\left\{0, \ldots, k-h_{0}\right\}$ such that $v_{h_{0}-1}^{c}>v_{h_{0}}^{c}=v_{h_{0}+\bar{h}}^{c}>$ $v_{h_{0}+\bar{h}+1}^{c}$, we have, for any $l=0, \ldots, \bar{h}$ :

$$
\sum_{h=h_{0}}^{h_{0}+l} \bar{p}^{\mathbf{x}}\left(h, v_{h}^{c}\right) \leq \sum_{h=h_{0}}^{h_{0}+l} \bar{p}^{\mathbf{y}}\left(h, v_{h}^{c}\right)
$$

and:

$$
\sum_{h=h_{0}+l}^{h_{0}+\bar{h}} p^{\mathbf{x}}\left(h, v_{h}^{c}\right)>\sum_{h=h_{0}+l}^{h_{0}+\bar{h}} p^{\mathbf{y}}\left(h, v_{h}^{c}\right)
$$

Proof. We first note that, for any sufficiently small strictly positive number $\varepsilon$, the vector of poverty lines:

$$
(v_{1}^{c}, \ldots, v_{h_{0}-1}^{c}, \underbrace{v_{h_{0}}^{c}+\varepsilon, \ldots, v_{h_{0}}^{c}+\varepsilon}_{\bar{h}-l}, \underbrace{\varepsilon-h_{0}}, \underbrace{v^{c}}_{h_{h_{0}}^{c}, \ldots, v_{h_{0}}^{c}}, v_{h_{0}+\bar{h}+1}^{c}, \ldots, v_{k}^{c})
$$

belongs to the set $\mathcal{V}$. Hence, since $x \succsim^{O P G} y$, we have:

$$
\begin{aligned}
& P^{\mathbf{x}}\left(\mathbf{v}^{c}\right)+\varepsilon\left[\sum_{h=h_{0}}^{h_{0}+l} \bar{p}^{\mathbf{x}}\left(h, v_{h}^{c}\right)\right] \\
= & P^{\mathbf{x}}\left(v_{1}^{c}, \ldots, v_{h_{0}-1}^{c}, v_{h_{0}}^{c}+\varepsilon, \ldots, v_{h_{0}}^{c}+\varepsilon, v_{h_{0}}^{c}, \ldots, v_{h_{0}}^{c}, v_{h_{0}+\bar{h}+1}^{c}, \ldots, v_{k}^{c}\right) \\
\leq & P^{\mathbf{y}}\left(v_{1}^{c}, \ldots, v_{h_{0}-1}^{c}, v_{h_{0}}^{c}+\varepsilon, \ldots, v_{h_{0}}^{c}+\varepsilon, v_{h_{0}}^{c}, \ldots, v_{h_{0}}^{c}, v_{h_{0}+\bar{h}+1}^{c}, \ldots, v_{k}^{c}\right) \\
= & P^{\mathbf{y}}\left(\mathbf{v}^{c}\right)+\varepsilon\left[\sum_{h=h_{0}}^{h_{0}+l} \bar{p}^{\mathbf{y}}\left(h, v_{h}^{c}\right)\right],
\end{aligned}
$$

which, when combined with the fact that $P^{\mathbf{x}}\left(v^{c}\right)-P^{\mathbf{y}}\left(v^{c}\right)=0$ by definition of $v^{c}$, implies inequality (A.7). Similarly the vector of poverty lines:

$$
(v_{1}^{c}, \ldots, v_{h_{0}-1}^{c}, \underbrace{v_{h_{0}}^{c}, \ldots, v_{h_{0}}^{c}}_{\bar{h}-l}, \underbrace{v_{h_{0}}^{c}-\varepsilon, \ldots, v_{h_{0}}^{c}-\varepsilon}, v_{h_{0}+\bar{h}+1}^{c}, \ldots, v_{k}^{c})
$$

${ }^{8}$ Using if necessary the convention that $v_{0}^{c}=\bar{v}(\mathbf{x}, \mathbf{y})$ and $v_{k+1}^{c}=\underline{v}(\mathbf{x}, \mathbf{y})$. 
belongs to the set $\mathcal{V}$ for a small enough $\varepsilon$. By the recursive definition of $v^{c}$, we have:

$$
\begin{aligned}
& =P^{\mathbf{x}}\left(v_{1}^{c}, \ldots, v_{h_{0}-1}^{c}, v_{h_{0}}^{c}, \ldots, v_{h_{0}}^{c}, v_{h_{0}}^{c}-\varepsilon, \ldots, v_{h_{0}}^{c}-\varepsilon, v_{h_{0}+\bar{h}+1}^{c}, \ldots, v_{k}^{c}\right) \\
& <P^{\mathbf{y}}\left(v_{1}^{c}, \ldots, v_{h_{0}-1}^{c}, v_{h_{0}}^{c}, \ldots, v_{h_{0}}^{c}, v_{h_{0}}^{c}-\varepsilon, \ldots, v_{h_{0}}^{c}-\varepsilon, v_{h_{0}+\bar{h}+1}^{c}, \ldots, v_{k}^{c}\right)
\end{aligned}
$$

and, therefore:

$$
\begin{aligned}
& P^{\mathbf{x}}\left(\mathbf{v}^{c}\right)-\varepsilon\left[\sum_{h=h_{0}+l}^{h_{0}+\bar{h}} p^{\mathbf{x}}\left(h, v_{h}^{c}\right)\right] \\
= & P^{\mathbf{x}}\left(v_{1}^{c}, \ldots, v_{h_{0}-1}^{c}, v_{h_{0}}^{c}, \ldots, v_{h_{0}}^{c}, v_{h_{0}}^{c}-\varepsilon, \ldots, v_{h_{0}}^{c}-\varepsilon, v_{h_{0}+\bar{h}+1}^{c}, \ldots, v_{k}^{c}\right) \\
< & P^{\mathbf{y}}\left(v_{1}^{c}, \ldots, v_{h_{0}-1}^{c}, v_{h_{0}}^{c}, \ldots, v_{h_{0}}^{c}, v_{h_{0}}^{c}-\varepsilon, \ldots, v_{h_{0}}^{c}-\varepsilon, v_{h_{0}+\bar{h}+1}^{c}, \ldots, v_{k}^{c}\right) \\
= & P^{\mathbf{y}}\left(\mathbf{v}^{c}\right)-\varepsilon\left[\sum_{h=h_{0}+l}^{h_{0}+\bar{h}} p^{\mathbf{y}}\left(h, v_{h}^{c}\right)\right],
\end{aligned}
$$

which, when combined with $P^{\mathbf{x}}\left(v^{c}\right)-P^{\mathbf{y}}\left(v^{c}\right)=0$, implies inequality (A.8).

Lemma A.5 has the following important corollary, that will be quite useful in establishing the possibility of making a nonzero BTPIT to the poorest individual in the worst category of distribution $\mathbf{y}$ when critical value $v_{1}^{c}$ is strictly larger than the income $\left(y_{1}^{1}\right)$ of this individual. This corollary in fact establishes the existence of (potential donors) individuals in a weakly larger category who have, in distribution $\mathbf{y}$, an income of $v_{1}^{c}$.

Corollary A.2. Let $\mathbf{x}$ and $\mathbf{y}$ be two distributions in $\mathcal{D}(I)$ such that $\mathbf{x} \succsim^{O P G} \mathbf{y}$. Suppose that $v_{1}^{c}>y_{1}^{1}$. Let $h_{0} \in\{0, \ldots, k-1\}$ be such that $v_{1}^{c}=v_{h_{0}+1}^{c}>v_{h_{0}+2}^{c}$ Then, there exists some $j \in$ $\left\{1, \ldots, h_{0}+1\right\}$ for which $y_{i}^{j}=v_{1}^{c}$, for some $i \in \mathcal{N}(j)$.

Proof. If $v_{1}^{c}>v_{2}^{c}$, we can apply Lemma A.5 to the case where $h_{0}=1$ and $\bar{h}=0$. In this case, Inequalities (A.7) and (A.8) write:

$$
\bar{p}^{\mathbf{x}}\left(1, v_{1}^{c}\right) \leq \bar{p}^{\mathbf{y}}\left(1, v_{1}^{c}\right)
$$

and:

$$
p^{\mathbf{x}}\left(1, v_{1}^{c}\right)>p^{\mathbf{y}}\left(1, v_{1}^{c}\right)
$$

Hence, there must exist an agent $i \in N(1)$ such that $y_{i}^{1}=v_{1}^{c}$. More generally, if $v_{1}^{c}=v_{2}^{c}=\cdots=$ $v_{k+1}^{c}>v_{k+2}^{c}$, one applies Lemma A.5 to the case where $h_{0}=1$ (taking $l=\bar{h}$ in (A.7) and $l=0$ in (A.8)), which gives

$$
\sum_{h=1}^{\bar{h}+1} \bar{p}^{\mathbf{x}}\left(1, v_{1}^{c}\right) \leq \sum_{h=1}^{\bar{h}+1} \bar{p}^{\mathbf{y}}\left(1, v_{1}^{c}\right)
$$


and:

$$
\sum_{h=1}^{\bar{h}+1} p^{\mathbf{x}}\left(1, v_{1}^{c}\right)>\sum_{h=1}^{\bar{h}+1} p^{\mathbf{y}}\left(1, v_{1}^{c}\right)
$$

One then obtains the existence of some $j \in\{1, \ldots, \bar{h}+1\}$ and some $i \in N(j)$ such that $y_{i}^{j}=$ $v_{j}^{c}=v_{1}^{c}$.

The next lemma shows that, when critical value $v_{1}^{c}$ is strictly larger than $y_{1}^{1}$, we in fact have some leeway to perform a BTPIT while preserving OPG dominance. Specifically, the following lemma deals with ordered vectors of poverty lines that assign to the worst category a poverty line only marginally above the lowest income observed in that category. This lemma says, roughly, that for any such ordered vector of poverty lines, the poverty gap in the dominated distribution must exceed that of the dominating one by an even larger margin. The precise statement of this lemma is as follows:

Lemma A.6. Let $\mathbf{x}$ and $\mathbf{y}$ be two distributions in $\mathcal{D}(I)$ such that $\mathbf{x} \succsim^{O P G} \mathbf{y}$. Suppose that $v_{1}^{c}>$ $y_{1}^{1}$. Then, for some strictly positive but suitably small real number $\varepsilon_{1}$, we have:

$$
P^{\mathbf{y}}\left(y_{1}^{1}+\varepsilon_{1}, v_{2}, \ldots, v_{k}\right) \geq P^{\mathbf{x}}\left(y_{1}^{1}+\varepsilon_{1}, v_{2}, \ldots, v_{k}\right)+\varepsilon_{1},
$$

provided $\left(y_{1}^{1}+\varepsilon_{1}, v_{2}, \ldots, v_{k}\right) \in \mathcal{V}$

Proof. Given $\mathbf{x}$ and $\mathbf{y}$ two distributions, define the income support of these two distributions:

$$
\mathcal{I}(\mathbf{x}, \mathbf{y})=\left\{a: \exists h \in\{1, \ldots, k\}, i \in\{1, \ldots, n(h)\} \text { such that } x_{i}^{h}=a \text { or } y_{i}^{h}=a\right\}
$$

Then choose the strictly positive number small enough as to satisfy:

$$
\varepsilon_{1}<\min _{a, b \in \mathcal{I}(\mathbf{x}, \mathbf{y}), a \neq b}|a-b|
$$

and

$$
\varepsilon_{1}<v_{1}^{c}-y_{1}^{1}
$$

Consider then any numbers $v_{2}, \ldots, v_{k}$ such that $v=\left(y_{1}^{1}+\varepsilon_{1}, v_{2}, \ldots, v_{k}\right) \in \mathcal{V}$ and let $h_{0} \in$ $\{1, \ldots, k\}$ be such that $v_{h}>y_{1}^{1}$ for all $h \in\left\{1, \ldots, h_{0}\right\}$ and $v_{h} \leq y_{1}^{1}$ for all $j \in\left\{h_{0}+1, \ldots, k\right\}$ (if there are such $j$ ). One can then write the vector $v$ as:

$$
\mathbf{v}=\left(y_{1}^{1}+\varepsilon_{1}, y_{1}^{1}+\varepsilon_{2}, \ldots, y_{1}^{1}+\varepsilon_{h_{0}}, v_{h_{0}+1}, v_{h_{0}+2}, \ldots, v_{k}\right)
$$

for some (possibly empty) list $\varepsilon_{2}, \ldots, \varepsilon_{h_{0}}$ satisfying $\varepsilon_{1} \geq \varepsilon_{2} \ldots \geq \varepsilon_{h_{0}}>0$. Let us prove that:

$$
P^{\mathbf{y}}(\mathbf{v}) \geq P^{\mathbf{x}}(\mathbf{v})+\varepsilon_{1} .
$$

Clearly, for $\varepsilon_{1}$ satisfying (A.9) and (A.10), we have:

$$
P^{\mathbf{y}}(\mathbf{v})=P^{\mathbf{y}}\left(y_{1}^{1}, \ldots, y_{1}^{1}, v_{h_{0}+1}, v_{h_{0}+2}, \ldots, v_{k}\right)+\sum_{h=1}^{h_{0}} \varepsilon_{h} \bar{p}^{\mathbf{y}}\left(h, y_{1}^{1}\right)
$$


and:

$$
P^{\mathbf{x}}(\mathbf{v})=P^{\mathbf{x}}\left(y_{1}^{1}, \ldots, y_{1}^{1}, v_{h_{0}+1}, v_{h_{0}+2}, \ldots, v_{k}\right)+\sum_{h=1}^{h_{0}} \varepsilon_{h} \bar{p}^{\mathbf{x}}\left(h, y_{1}^{1}\right)
$$

and, therefore:

$$
\begin{gathered}
P^{\mathbf{y}}(\mathbf{v})-P^{\mathbf{x}}(\mathbf{v})=P^{\mathbf{y}}\left(y_{1}^{1}, \ldots, y_{1}^{1}, v_{h_{0}+1}, \ldots, v_{k}\right)-P^{\mathbf{x}}\left(y_{1}^{1}, \ldots, y_{1}^{1}, v_{h_{0}+1}, \ldots, v_{k}\right) \\
+\sum_{h=1}^{h_{0}} \varepsilon_{h}\left[\bar{p}^{\mathbf{y}}\left(h, y_{1}^{1}\right)-\bar{p}^{\mathbf{x}}\left(h, y_{1}^{1}\right)\right] .
\end{gathered}
$$

If

$$
P^{\mathbf{y}}\left(y_{1}^{1}, \ldots, y_{1}^{1}, v_{h_{0}+1}, \ldots, v_{k}\right)>P^{\mathbf{x}}\left(y_{1}^{1}, \ldots, y_{1}^{1}, v_{h_{0}+1}, \ldots, v_{k}\right)
$$

then there is nothing to prove. Indeed, from Lemma A.1 and the assumption that $y_{i}^{1} \neq x_{i}^{1}$ for all $i \in \mathcal{N}(1)$, we have that $\bar{p}^{\mathbf{y}}\left(1, y_{1}^{1}\right) \geq 1$ and $\bar{p}^{\mathbf{x}}\left(1, y_{1}^{1}\right)=0$. Hence:

$$
\begin{gathered}
P^{\mathbf{y}}\left(y_{1}^{1}, \ldots, y_{1}^{1}, v_{h_{0}+1}, v_{h_{0}+2}, \ldots, v_{k}\right)-P^{\mathbf{x}}\left(y_{1}^{1}, \ldots, y_{1}^{1}, v_{h_{0}+1}, v_{h_{0}+2}, \ldots, v_{k}\right) \\
+\varepsilon_{1}\left[\bar{p}^{\mathbf{y}}\left(1, y_{1}^{1}\right)-\bar{p}^{\mathbf{x}}\left(1, y_{1}^{1}\right)\right] \\
>\varepsilon_{1}
\end{gathered}
$$

for any $\varepsilon_{1}$ satisfying (A.9) and (A.10). Because of this, we can choose $\varepsilon_{1}$ sufficiently small so as make the numbers $\varepsilon_{2}, \ldots, \varepsilon_{h_{0}}$ sufficiently small for the inequality:

$$
\begin{aligned}
& P^{\mathbf{y}}\left(y_{1}^{1}, \ldots, y_{1}^{1}, v_{h_{0}+1}, v_{h_{0}+2}, \ldots, v_{k}\right)-P^{\mathbf{x}}\left(y_{1}^{1}, \ldots, y_{1}^{1}, v_{h_{0}+1}, v_{h_{0}+2}, \ldots, v_{k}\right) \\
& +\varepsilon_{1}\left[\bar{p}^{\mathbf{y}}\left(1, y_{1}^{1}\right)-\bar{p}^{\mathbf{x}}\left(1, y_{1}^{1}\right)\right]+\sum_{h=2}^{h_{0}} \varepsilon_{h}\left[\bar{p}^{\mathbf{y}}\left(h, y_{1}^{1}\right)-\bar{p}^{\mathbf{x}}\left(h, y_{1}^{1}\right)\right] \\
& \geq \varepsilon_{1}
\end{aligned}
$$

to hold. Suppose now that:

$$
P^{\mathbf{y}}\left(y_{1}^{1}, \ldots, y_{1}^{1}, v_{h_{0}+1}, v_{h_{0}+2}, \ldots, v_{k}\right)=P^{\mathbf{x}}\left(y_{1}^{1}, \ldots, y_{1}^{1}, v_{h_{0}+1}, v_{h_{0}+2}, \ldots, v_{k}\right) .
$$

In that case, it follows from (A.11) that:

$$
P^{\mathbf{y}}(\mathbf{v})-P^{\mathbf{x}}(\mathbf{v})=\sum_{h=1}^{h_{0}} \varepsilon_{h}\left[\bar{p}^{\mathbf{y}}\left(h, y_{1}^{1}\right)-\bar{p}^{\mathbf{x}}\left(h, y_{1}^{1}\right)\right] .
$$

This equality can equivalently be written as:

$$
P^{\mathbf{y}}(\mathbf{v})-P^{\mathbf{x}}(\mathbf{v})=\sum_{h=1}^{h_{0}}\left[\varepsilon_{h}-\varepsilon_{h+1}\right] \sum_{g=1}^{h}\left[\bar{p}^{\mathbf{y}}\left(g, y_{1}^{1}\right)-\bar{p}^{\mathbf{x}}\left(g, y_{1}^{1}\right)\right]
$$


using the convention that $\varepsilon_{h_{0}+1}=0$. Note that, by definition of $v_{1}^{c}$, we must have $P^{\mathbf{y}}(\mathbf{v})-$ $P^{\mathbf{x}}(\mathbf{v})>0$ if $\varepsilon_{1}$ satisfies (A.10). Note also that, for all $h \in\left\{1, \ldots, h_{0}\right\}$, we have:

$$
\sum_{g=1}^{h}\left[\bar{p}^{\mathbf{y}}\left(g, y_{1}^{1}\right)-\bar{p}^{\mathbf{x}}\left(g, y_{1}^{1}\right)\right] \geq 1 .
$$

Indeed, by definition of $v_{1}^{c}$, we have for every strictly positive $\delta \leq \varepsilon_{1}$ :

$$
\begin{gathered}
P^{\mathbf{y}}(\underbrace{y_{1}^{1}+\delta, \ldots, y_{1}^{1}+\delta}_{h}, \underbrace{y_{1}^{1}, \ldots, y_{1}^{1}}_{h_{0}-h}, v_{h_{0}+1}, v_{h_{0}+2}, \ldots, v_{k}) \\
>P^{\mathbf{x}}(\underbrace{y_{1}^{1}+\delta, \ldots, y_{1}^{1}+\delta}_{h}, \underbrace{y_{1}^{1}, \ldots, y_{1}^{1}}_{h_{0}-h}, v_{h_{0}+1}, v_{h_{0}+2}, \ldots, v_{k}) .
\end{gathered}
$$

Yet,

$$
\begin{aligned}
& P^{\mathbf{y}}(\underbrace{y_{1}^{1}+\delta, \ldots, y_{1}^{1}+\delta}_{h}, \underbrace{y_{1}^{1}, \ldots, y_{1}^{1}}_{h_{0}-h}, v_{h_{0}+1}, v_{h_{0}+2}, \ldots, v_{k}) \\
= & P^{\mathbf{y}}\left(y_{1}^{1}, \ldots, y_{1}^{1}, v_{h_{0}+1}, v_{h_{0}+2}, \ldots, v_{k}\right)+\delta \sum_{g=1}^{h} \bar{p}^{\mathbf{y}}\left(g, y_{1}^{1}\right)
\end{aligned}
$$

and:

$$
\begin{aligned}
& P^{\mathbf{x}}(\underbrace{y_{1}^{1}+\delta, \ldots, y_{1}^{1}+\delta}_{h}, \underbrace{y_{1}^{1}, \ldots, y_{1}^{1}}_{h_{0}-h}, v_{h_{0}+1}, v_{h_{0}+2}, \ldots, v_{k}) \\
= & P^{\mathbf{x}}\left(x_{1}^{1}, \ldots, x_{1}^{1}, v_{h_{0}+1}, v_{h_{0}+2}, \ldots, v_{k}\right)+\delta \sum_{g=1}^{h} \bar{p}^{\mathbf{x}}\left(g, y_{1}^{1}\right) .
\end{aligned}
$$

Hence, under the assumption that:

$$
P^{\mathbf{y}}\left(y_{1}^{1}, \ldots, y_{1}^{1}, v_{h_{0}+1}, v_{h_{0}+2}, \ldots, v_{k}\right)=P^{\mathbf{x}}\left(y_{1}^{1}, \ldots, y_{1}^{1}, v_{h_{0}+1}, v_{h_{0}+2}, \ldots, v_{k}\right)
$$

we have:

$$
\begin{aligned}
& P^{\mathbf{y}}\left(y_{1}^{1}+\delta, \ldots, y_{1}^{1}+\delta, y_{1}^{1}, \ldots, y_{1}^{1}, v_{h_{0}+1}, v_{h_{0}+2}, \ldots, v_{k}\right) \\
& -P^{\mathbf{x}}\left(y_{1}^{1}+\delta, \ldots, y_{1}^{1}+\delta, y_{1}^{1}, \ldots, y_{1}^{1}, v_{h_{0}+1}, v_{h_{0}+2}, \ldots, v_{k}\right) \\
= & \delta \sum_{g=1}^{h}\left[\bar{p}^{\mathbf{y}}\left(g, y_{1}^{1}\right)-\bar{p}^{\mathbf{x}}\left(g, y_{1}^{1}\right)\right]>0,
\end{aligned}
$$

which establishes Inequality (A.13). Together with (A.12), this leads to the conclusion that:

$$
P^{\mathbf{y}}(\mathbf{v})-P^{\mathbf{x}}(\mathbf{v}) \geq \varepsilon_{1}-\epsilon_{h_{0}+1}=\varepsilon_{1},
$$


as required.

Proof of Proposition 1. There exists some $h_{0} \in\{1, \ldots, k\}$ such that $v_{1}^{c}=v_{2}^{c}=\cdots v_{h_{0}}^{c}>$ $v_{h_{0}+1}^{c}$ (using, if necessary, the convention that $v_{k+1}^{c}=\underline{v}(\mathbf{x}, \mathbf{y})$ ). Then, using Corollary A.2, we conclude that there is some category $j \in\left\{1, \ldots, h_{0}\right\}$ and some individual $i^{j} \in \mathcal{N}(j)$ such that $y_{i j}^{j}=v_{1}^{c}$ and $\forall h \in\left\{j, j+1, \ldots, h_{0}\right\}, i \in \mathcal{N}(h), y_{i}^{h} \neq v_{1}^{c}$ (i.e., $j$ is the highest category in the set $\left\{1, \ldots, h_{0}\right\}$ for which there is an individual in distribution $\mathbf{y}$ whose income is equal to $v_{1}^{c}$.). It is important to note that we do not preclude the possibility that $j=1$. Let us show that there exists a distribution $\widehat{\mathbf{x}} \in \mathcal{D}(I)$ such that $\mathbf{x} \succsim^{O P G} \widehat{\mathbf{x}}$ and $\widehat{\mathbf{x}}$ is obtained from $\mathbf{y}$ by a BTPIT. For any strictly positive integer $m$, let $\widehat{\mathbf{x}}^{m}$ be the distribution obtained from distribution $\mathbf{y}$ by performing a BTPIT of an amount of $1 / m$ from agent $i^{j} \in \mathcal{N}(j)$ to agent $1 \in \mathcal{N}(1)$. We claim that there exists some $m$ sufficiently large that $\mathbf{x} \succsim O P G \widehat{\mathbf{x}}^{m}$. Assume by contradiction that no such $m$ exists. This implies the existence of a sequence of ordered poverty lines vectors $\mathbf{v}^{m} \in \mathcal{V}^{\prime}$ such that $P^{\widehat{\mathbf{x}}^{m}}\left(\mathbf{v}^{m}\right)<P^{\mathbf{x}}\left(\mathbf{v}^{m}\right)$. Note that, for every strictly positive real integer $m$, and whatever the ordered vector of poverty lines $\mathbf{v} \in \mathcal{V}^{\prime}$ is, we have:

$$
P^{\widehat{\mathbf{x}}^{m}}(\mathbf{v})=P^{\mathbf{y}}(\mathbf{v})-1 / m \text { if } v_{1} \geq y_{1}^{1}+1 / m \text { and } v_{j} \leq y_{i^{j}}^{j}-1 / m,
$$

$$
=P^{\mathbf{y}}(\mathbf{v})-\max \left(v_{1}-y_{1}^{1}, 0\right) \text { if } v_{1}<y_{1}^{1}+\frac{1}{m}, v_{j} \leq y_{i^{j}}^{j}-\frac{1}{m}
$$

$$
=P^{\mathbf{y}}(\mathbf{v})+\min \left(v_{j}-y_{i^{j}}^{j}, 0\right) \text { if } v_{1} \geq y_{1}^{1}+\frac{1}{m}, v_{j}>y_{i^{j}}^{j}-\frac{1}{m} .
$$

Because of this, we can assume without loss of generality that $v_{1}^{m} \geq y_{1}^{1}+1 / m$ and $v_{j}^{m} \leq v_{1}^{c}-$ $1 / m$. By compactness of $\mathcal{V}^{\prime}, \mathbf{v}^{m}$ admits a subsequence that converges to some vector of ordered poverty lines $\mathbf{v} \in \mathcal{V}^{\prime}$. By continuity, we must have $P^{\mathbf{y}}(\mathbf{v})=P^{\mathbf{x}}(\mathbf{v})$. Hence, by definition of critical value $v_{1}^{c}$,

$$
\text { either: (i) } v_{1}=y_{1}^{1} \text { or (ii) }
$$

$v_{1} \geq v_{1}^{c}$

- If case (i) holds, then we have:

$$
\begin{aligned}
P^{\widehat{\mathbf{x}}^{m}}\left(\mathbf{v}^{m}\right) & \geq P^{\mathbf{y}}\left(\mathbf{v}^{m}\right)-1 / m \text { by (A.14)-(A.16) } \\
& \geq P^{\mathbf{x}}\left(\mathbf{v}^{m}\right)-1 / m+v_{1}^{m}-y_{1}^{1} \text { by Lemma } A .6, \text { taking } v_{1}^{m}-y_{1}^{1}=\varepsilon_{1} \\
& \geq P^{\mathbf{x}}\left(\mathbf{v}^{m}\right),
\end{aligned}
$$

which is a contradiction.

- Now, if case (ii) holds and $v_{1} \geq v_{1}^{c}$ then by Corollary A.1, we must have $v_{h} \geq v_{h}^{c}$ for $h=$ $2, \ldots, k$. In particular, since $v_{j}^{m} \leq v_{1}^{c}-1 / m$ and $\mathbf{v}^{m}$ admits a subsequence that converges to $\mathbf{v}$, we must have $v_{j}=v_{j}^{c}=v_{1}^{c}$. We can actually assume without loss of generality that, for every $h=1, \ldots, h_{0}, v_{h}^{m} \in\left\{v_{1}^{c}-1 / m, v_{1}^{c}\right\}$ (for large enough $m, v_{1}^{c}-1 / m$ and $v_{1}^{c}$ are the only two incomes observed in distributions $\mathbf{y}, \widehat{\mathbf{x}}^{m}$ and $\mathbf{x}$ for poverty lines $v_{h}^{m}$ relevant for categories $\left.h=1, \ldots, h_{0}\right)$. Hence, for some $g \in\{1, \ldots, j\}$, we have: $v_{g}^{m}=\cdots=v_{j}^{m}=$ $\cdots v_{h^{0}}^{m}=v_{1}^{c}-1 / m, v_{1}^{m}=v_{2}^{m}=\cdots=v_{g-1}^{m}=v_{1}^{c}$. Since:

$$
\sum_{h=g}^{h_{0}} p^{\mathbf{x}}\left(h, v_{1}^{c}\right)>\sum_{h=g}^{h_{0}} p^{\mathbf{y}}\left(h, v_{1}^{c}\right)
$$


we have:

$$
\begin{aligned}
& \sum_{h=g}^{h_{0}} \sum_{i \in \mathcal{P}^{y}\left(h, v_{h}^{m}\right)}\left[v_{h}^{m}-y_{i}^{h}\right]-\sum_{h=g}^{h_{0}} \sum_{i \in \mathcal{P}^{x}\left(h, v_{h}^{m}\right)}\left[v_{h}^{m}-x_{i}^{h}\right]-1 / m \\
\geq & \sum_{h=g}^{h_{0}} \sum_{i \in \mathcal{P}^{y}\left(h, v_{1}^{c}\right)}\left[v_{1}^{c}-y_{i}^{h}\right]-\sum_{h=g}^{h_{0}} \sum_{i \in \mathcal{P}^{x}\left(h, v_{1}^{c}\right)}\left[v_{1}^{c}-x_{i}^{h}\right]
\end{aligned}
$$

and, therefore:

$$
\begin{aligned}
& P^{\mathbf{y}}\left(\mathbf{v}^{m}\right)-P^{\mathbf{x}}\left(\mathbf{v}^{m}\right)-1 / m \\
\geq & P^{\mathbf{y}}\left(v_{1}^{c}, \ldots, v_{1}^{c}, v_{h_{0}+1}^{c}, \ldots, v_{k}^{c}\right)-P^{\mathbf{x}}\left(v_{1}^{c}, \ldots, v_{1}^{c}, v_{h_{0}+1}^{c}, \ldots, v_{k}^{c}\right) \geq 0 .
\end{aligned}
$$

Finally $P^{\widehat{\mathbf{x}}^{m}}\left(\mathbf{v}^{m}\right) \geq P^{\mathbf{y}}\left(\mathbf{v}^{m}\right)-1 / m \geq P^{\mathbf{x}}\left(\mathbf{v}^{m}\right)$, a contradiction.

Proof of the claim in Example 2. Consider distribution $\widehat{\mathbf{x}}^{\varepsilon}$ defined by:

$$
\widehat{x}_{1}^{\varepsilon 1}=3+\varepsilon, \widehat{x}_{2}^{\varepsilon 2}=4-\varepsilon, \widehat{x}_{2}^{\varepsilon 1}=\widehat{x}_{2}^{1}=7 \text { and } \widehat{x}_{1}^{\varepsilon 2}=\widehat{x}_{1}^{2}=0 .
$$

Then, using the ordered vector $\mathbf{v}=(3+\varepsilon, 3+\varepsilon)$ of poverty lines, we have:

$$
\begin{aligned}
P^{\widehat{\mathbf{x}}^{\varepsilon}}(3+\varepsilon, 3+\varepsilon)= & \max (3+\varepsilon-(3+\varepsilon), 0)+\max (3+\varepsilon-7,0) \\
& +\max (3+\varepsilon-0,0)+\max (3+\varepsilon-(4-\varepsilon), 0) \\
= & 3+\varepsilon(\text { if } \varepsilon \in] 0,1 / 2]) \\
< & P^{\mathbf{x}}(3+\varepsilon, 3+\varepsilon) \\
= & \max (3+\varepsilon-5,0)+\max (3+\varepsilon-6,0) \\
& +\max (3+\varepsilon-1,0)+\max (3+\varepsilon-2,0) \\
= & 3+2 \varepsilon .
\end{aligned}
$$

Proof of Proposition 2. We prove this proposition using Lemma A.3. We note first that, since $x_{i}^{1}>v_{1}^{c} \forall i \in \mathcal{N}(1)$, we must have $v_{1}^{c}=v_{2}^{c}$. Suppose by contradiction that $v_{1}^{c}>v_{2}^{c}$. This means that, for any number $\varepsilon$ such that $v_{1}^{c}-v_{2}^{c}>\varepsilon>0$, we have that $\left(v_{1}^{c}-\varepsilon, v_{2}^{c}, \ldots, v_{k}^{c}\right) \in \mathcal{V}$ and

$$
\begin{aligned}
& P^{\mathbf{y}}\left(v_{1}^{c}-\varepsilon, v_{2}^{c}, \ldots, v_{k}^{c}\right)-P^{\mathbf{x}}\left(v_{1}^{c}-\varepsilon, v_{2}^{c}, \ldots, v_{k}^{c}\right) \\
= & -\varepsilon p^{\mathbf{y}}\left(1, v_{1}^{c}\right)+\varepsilon p^{\mathbf{x}}\left(1, v_{1}^{c}\right)+P^{\mathbf{y}}\left(v_{1}^{c}, v_{2}^{c}, \ldots, v_{k}^{c}\right)-P^{\mathbf{x}}\left(v_{1}^{c}, v_{2}^{c}, \ldots, v_{k}^{c}\right) \\
= & -\varepsilon p^{\mathbf{y}}\left(1, v_{1}^{c}\right) \\
< & 0
\end{aligned}
$$

since $p^{\mathbf{x}}\left(1, v_{1}^{c}\right)=0=P^{\mathbf{y}}\left(v_{1}^{c}, v_{2}^{c}, \ldots, v_{k}^{c}\right)-P^{\mathbf{x}}\left(v_{1}^{c}, v_{2}^{c}, \ldots, v_{k}^{c}\right) \quad$ (by definition of $\left.\mathbf{v}^{c}\right)$ and $p^{\mathbf{y}}\left(1, v_{1}^{c}\right) \geq 1$. But this is a contradiction of the fact that $\mathbf{x} \succsim^{O P G} \mathbf{y}$. It then follows that $h_{0} \geq 2$ and that the second bullet statement of the proposition (e.g., "For any category $h$ such that $h_{0} \geq h \geq 2$, we have $y_{i}^{h} \neq v_{1}^{c}$ ") is not empty. This fact and Corollary A.2 establish the existence 
of an agent $i_{1} \in \mathcal{N}(1)$ for which $y_{i_{1}}^{1}=v_{1}^{c}$. Hence vector of poverty lines $\mathbf{v}^{c}$ is just like vector $\mathbf{w}$ in the antecedent clause of Lemma A.3. It then follows from Lemma A.5 that:

$$
\sum_{h=l+1}^{h_{0}} \bar{p}^{\mathbf{y}}\left(h, y_{i_{1}}^{1}\right)=\sum_{h=l+1}^{h_{0}} p^{\mathbf{y}}\left(h, y_{i_{1}}^{1}\right)<\sum_{h=l+1}^{h_{0}} p^{\mathbf{x}}\left(h, y_{i_{1}}^{1}\right) \leq \sum_{h=l+1}^{h_{0}} \bar{p}^{\mathbf{x}}\left(h, y_{i_{1}}^{1}\right)
$$

for $l=1, \ldots, h_{0}-1$, which implies that Inequality (A.4) in the antecedent clause of Lemma A.3 holds. The existence of a distribution $\widehat{\mathbf{x}} \in \mathcal{D}(I)$ such that $\widehat{\mathbf{x}}$ is obtained from $\mathbf{y}$ by an FIP and $\mathbf{x} \succsim^{O P G} \widehat{\mathbf{x}}$ then immediately follows from this lemma.

A.6 Case (B) Proof of Proposition 3.

We start the analysis of this case by observing that requiring (5) to hold for all lists of poverty lines in the set $\mathcal{V}$ is equivalent to requiring this inequality to hold for the subset

$$
\mathcal{V}^{\prime}=\left\{\left(v_{1}, \ldots, v_{k}\right) \in \mathbb{R}^{k}: \bar{v}(\mathbf{x}, \mathbf{y}) \geq v_{1} \geq v_{2} \geq \ldots \geq v_{k} \geq \underline{v}(\mathbf{x}, \mathbf{y})\right\}
$$

of such lists of poverty lines, which is compact.

We also observe that, by the very definition of critical value $v_{1}^{c}$, there exists a sequence $\left\{\mathbf{w}^{m}\right\}$ of poverty lines vectors (with $\mathbf{w}^{m} \in \mathcal{V}^{\prime}$ for every $m$ ) such that $P^{\mathbf{y}}\left(\mathbf{w}^{m}\right)-P^{\mathbf{x}}\left(\mathbf{w}^{m}\right)=0$ and $w_{1}^{m}=$ $y_{1}^{1}+\varepsilon_{1}^{m}$, for $\varepsilon_{1}^{m}>0$, and $\varepsilon_{1}^{m} \rightarrow 0$. By compactness of $\mathcal{V}^{\prime}$ we can assume without loss of generality ${ }^{9}$ that the sequence $\mathbf{w}^{m}$ of ordered poverty lines vectors converges to some limit $\overline{\mathbf{w}} \in \mathcal{V}^{\prime}$. By continuity of the poverty gap function $P$, we have $P^{\mathbf{y}}(\overline{\mathbf{w}})-P^{\mathbf{x}}(\overline{\mathbf{w}})=0$.

Taking this limit vector $\overline{\mathbf{w}} \in \mathcal{V}^{\prime}$ of ordered poverty lines, we first establish the existence, in initial distribution $\mathbf{y}$, of some agent in a category strictly larger than 1 with an income strictly larger than the lowest income observed in category 1 . This agent will be a natural candidate for exchanging his/her higher income with that of the poorest agent in category 1. A crucial step for the identification of such an agent is the following lemma.

Lemma A.7. Let $\mathbf{x}$ and $\mathbf{y}$ be two distributions in $\mathcal{D}(I)$ such that $\mathbf{x} \succsim^{O P G} \mathbf{y}$. Suppose that $v_{1}^{c}=$ $y_{1}^{1}$. Then, there exists $h_{0} \geq 2$ such that $y_{1}^{1}=\bar{w}_{1}=\bar{w}_{2}=\cdots=\bar{w}_{h_{0}}>\bar{w}_{h_{0}+1}$. Moreover there exists $g_{0} \leq h_{0}$ such that $g_{0} \geq 2$,

$$
\sum_{h=1}^{g_{0}} \bar{p}^{\mathbf{y}}\left(h, y_{1}^{1}\right)=\sum_{h=1}^{g_{0}} \bar{p}^{\mathbf{x}}\left(h, y_{1}^{1}\right)
$$

and:

$$
\sum_{h=1}^{l} \bar{p}^{\mathbf{y}}\left(h, y_{1}^{1}\right)>\sum_{h=1}^{l} \bar{p}^{\mathbf{x}}\left(h, y_{1}^{1}\right)
$$

for all $l<g_{0}$.

Proof. Let $\overline{\mathbf{w}}$ be the vector of ordered poverty lines that is the limit of the sequence $\left\{w^{m}\right\}$ of ordered poverty lines vectors satisfying $w_{1}^{m}=y_{1}^{1}+\varepsilon_{1}^{m}$ with $\varepsilon_{1}^{m} \rightarrow 0$ and $P^{\mathbf{x}}\left(w^{m}\right)-$ $P^{\mathbf{y}}\left(w^{m}\right)=0$ for all $m$ that was mentioned in Case (B) of Subsection 3.1. We first show that $\bar{w}_{1}=\bar{w}_{2}$. By contradiction, suppose that $\bar{w}_{2}<\bar{w}_{1}$. Then, there exists a large enough $m$ for which $w_{2}^{m}<\bar{w}_{1}=y_{1}^{1}$. Also, for a large enough $m$, we have that:

$$
w_{1}^{m} \bar{p}^{\mathbf{x}}\left(1, w_{1}^{m}\right)-\sum_{i \in \overline{\mathcal{P}}^{\mathrm{x}}\left(1, w_{1}^{m}\right)} x_{i}^{1}=w_{1} \bar{p}^{\mathbf{x}}\left(1, y_{1}^{1}\right)-\sum_{i \in \overline{\mathcal{P}}^{\mathrm{x}}\left(1, y_{1}^{1}\right)} x_{i}^{1}=0
$$

${ }^{9}$ Taking a subsequence if necessary. 
thanks to Lemma A.1 and the fact that $y_{1}^{1} \neq x_{1}^{1}$. Moreover we have:

$$
\begin{aligned}
w_{1}^{m} \bar{p}^{\mathbf{y}}\left(1, w_{1}^{m}\right)-\sum_{i \in \overline{\mathcal{P}}^{\mathbf{y}}\left(1, w_{1}^{m}\right)} y_{i}^{1} & =\left(\varepsilon_{1}^{m}+y_{1}^{1}\right) \bar{p}^{\mathbf{y}}\left(1, y_{1}^{1}\right)-\sum_{i \in \overline{\mathcal{P}}^{\mathbf{y}}\left(1, w_{1}\right)} y_{i}^{1} \\
& >y_{1}^{1} \bar{p}^{\mathbf{y}}\left(1, y_{1}^{1}\right)-\sum_{i \in \overline{\mathcal{P}}^{\mathbf{y}}\left(1, w_{1}\right)} y_{i}^{1}=0
\end{aligned}
$$

because $\bar{p}^{\mathrm{y}}\left(1, y_{1}^{1}\right) \geq 1$. Hence:

$$
\begin{aligned}
& P^{\mathbf{y}}\left(\mathbf{w}^{m}\right)-P^{\mathbf{x}}\left(\mathbf{w}^{m}\right)=w_{1}^{m} \bar{p}^{\mathbf{y}}\left(1, w_{1}^{m}\right)-\sum_{i \in \overline{\mathcal{P}}^{\mathbf{y}}\left(1, w_{1}^{m}\right)} y_{i}^{1}+\sum_{h=2}^{k} P^{\mathbf{y}}\left(h, w_{h}^{m}\right) \\
& -\left[w_{1}^{m} \bar{p}^{\mathbf{x}}\left(1, w_{1}^{m}\right)-\sum_{i \in \overline{\mathcal{P}}^{\mathbf{x}}\left(1, w_{1}^{m}\right)} x_{i}^{1}+\sum_{h=2}^{k} P^{\mathbf{x}}\left(h, w_{h}^{m}\right)\right] \\
& =0 \\
& >y_{1}^{1} \bar{p}^{\mathbf{y}}\left(1, y_{1}^{1}\right)-\sum_{i \in \overline{\mathcal{P}}^{\mathbf{y}}\left(1, w_{1}\right)} y_{i}^{1}+\sum_{h=2}^{k} P^{\mathbf{y}}\left(h, w_{h}^{m}\right) \\
& -\left[w_{1} \bar{p}^{\mathbf{x}}\left(1, y_{1}^{1}\right)-\sum_{i \in \overline{\mathcal{P}}^{\mathbf{x}}\left(1, y_{1}^{1}\right)} x_{i}^{1}+\sum_{h=2}^{k} P^{\mathbf{x}}\left(h, w_{h}^{m}\right)\right] \\
& =P^{\mathbf{y}}\left(y_{1}^{1}, w_{2}^{m}, \ldots, w_{k}^{m}\right)-P^{\mathbf{x}}\left(y_{1}^{1}, w_{2}^{m}, \ldots, w_{k}^{m}\right)
\end{aligned}
$$

a contradiction. We now show that $w_{2}^{m}>y_{1}^{1}$. Indeed, for $m$ large enough, we have:

$$
P^{\mathbf{y}}\left(\mathbf{w}^{m}\right)=P^{\mathbf{y}}\left(y_{1}^{1}, w_{2}^{m}, \ldots, w_{k}^{m}\right)+\varepsilon_{1}^{m} p^{\mathbf{y}}\left(1, y_{1}^{1}\right)
$$

and:

$$
P^{\mathbf{x}}\left(\mathbf{w}^{m}\right)=P^{\mathbf{x}}\left(y_{1}^{1}, w_{2}^{m}, \ldots, w_{k}^{m}\right)+\varepsilon_{1}^{m} p^{\mathbf{x}}\left(1, y_{1}^{1}\right)
$$

Moreover, by Lemma A. 1 and because, $y_{i}^{1} \neq x_{i}^{1}$, we have that

$$
\bar{p}^{\mathbf{y}}\left(1, y_{1}^{1}\right) \geq 1>0=p^{\mathbf{x}}\left(1, y_{1}^{1}\right) .
$$

Hence, we have:

$$
\begin{aligned}
P^{\mathbf{y}}\left(\mathbf{w}^{m}\right)-P^{\mathbf{x}}\left(\mathbf{w}^{m}\right) & =0 \\
& \geq P^{\mathbf{y}}\left(y_{1}^{1}, w_{2}^{m}, \ldots, w_{k}^{m}\right)-P^{\mathbf{x}}\left(y_{1}^{1}, w_{2}^{m}, \ldots, w_{k}^{m}\right)+\varepsilon_{1}^{m} .
\end{aligned}
$$

Because of this (and the fact that $\varepsilon_{1}^{m}>0$ ), assuming that $w_{2}^{m} \leq y_{1}^{1}$ and, therefore, that the vector of poverty lines $\left(y_{1}^{1}, w_{2}^{m}, \ldots, w_{k}^{m}\right)$ belongs to $\mathcal{V}$ would be contradictory with the fact that $x \succsim^{O P G} y$. We also know that $w_{h_{0}+1}^{m}<y_{1}^{1}$. Let $l:=\min \left\{h \geq 1: w_{h}^{m} \leq y_{1}^{1}\right\}$. As we have just shown $3 \leq l \leq h_{0}+1$. For $h=1, \ldots, l$, we have $w_{h}^{m}=y_{1}^{1}+\varepsilon_{h}^{m}$ with $\varepsilon_{1}^{m} \geq \varepsilon_{2}^{m} \geq \ldots \geq \varepsilon_{l}^{m}>$ 
0 . We know already that $\bar{p}^{\mathrm{y}}\left(1, y_{1}^{1}\right) \geq 1>0=\bar{p}^{\mathrm{x}}\left(1, y_{1}^{1}\right)$. Suppose that the main claim of the lemma was false. In that case, we would have:

$$
\sum_{g=1}^{h} p^{\mathbf{y}}\left(g, y_{1}^{1}\right)>\sum_{g=1}^{h} p^{\mathbf{x}}\left(g, y_{1}^{1}\right)
$$

for all $h=1, \ldots, h_{0}$. Yet:

$$
\begin{aligned}
0 & =P^{\mathbf{y}}\left(\mathbf{w}^{m}\right)-P^{\mathbf{x}}\left(\mathbf{w}^{m}\right) \\
& =P^{\mathbf{y}}\left(y_{1}^{1}, \ldots, y_{1}^{1}, w_{l+1}^{m}, \ldots, w_{k}^{m}\right)-P^{\mathbf{x}}\left(y_{1}^{1}, \ldots, y_{1}^{1}, w_{l+1}^{m}, \ldots, w_{k}^{m}\right) \\
& +\sum_{h=1}^{l}\left[\bar{p}^{\mathbf{y}}\left(h, y_{1}^{1}\right)-\bar{p}^{\mathbf{x}}\left(h, y_{1}^{1}\right)\right] \varepsilon_{h}^{m} \\
& =P^{\mathbf{y}}\left(y_{1}^{1}, \ldots, y_{1}^{1}, w_{s+1}^{m}, \ldots, w_{k}^{m}\right)-P^{\mathbf{x}}\left(y_{1}^{1}, \ldots, y_{1}^{1}, w_{l+1}^{m}, \ldots, w_{k}^{m}\right) \\
& +\sum_{h=1}^{l-1}\left(\varepsilon_{h}^{m}-\varepsilon_{h+1}^{m}\right) \sum_{g=1}^{h}\left[\bar{p}^{\mathbf{y}}\left(g, y_{1}^{1}\right)-\bar{p}^{\mathbf{x}}\left(g, y_{1}^{1}\right)\right]+\varepsilon_{l}^{m} \sum_{g=1}^{l}\left[p^{\mathbf{y}}\left(g, y_{1}^{1}\right)-p^{\mathbf{x}}\left(g, y_{1}^{1}\right)\right] \\
& \geq \sum_{h=1}^{s-1}\left(\varepsilon_{h}^{m}-\varepsilon_{h+1}^{m}\right) \sum_{g=1}^{h}\left[\bar{p}^{\mathbf{y}}\left(g, y_{1}^{1}\right)-\bar{p}^{\mathbf{x}}\left(g, y_{1}^{1}\right)\right]+\varepsilon_{l}^{m} \sum_{g=1}^{l}\left[\bar{p}^{\mathbf{y}}\left(g, y_{1}^{1}\right)-\bar{p}^{\mathbf{x}}\left(g, y_{1}^{1}\right)\right] \\
\geq & \varepsilon_{l}^{m}>0
\end{aligned}
$$

a contradiction.

This lemma indeed identifies a category $g_{0}$ strictly larger than 1 in which a "potential donor" to the poorest agent in the worst category can be selected. As we now establish, this donor can transfer to the poorest agent in category 1 the whole income difference, while maintaining the dominance of distribution $\mathbf{x}$ over the distribution created by the FIP.

Proof of Proposition 3. We base the argument on Lemma A.3. We must therefore prove that the limit vector of poverty lines $\overline{\mathbf{w}}$ satisfies the conditions imposed on vector $\mathbf{w}$ of this lemma. From Lemma A.7, we have that $y_{1}^{1}=\bar{w}_{1}=\bar{w}_{2}=\cdots=\bar{w}_{h_{0}}>\bar{w}_{h_{0}}+1$ for some $h_{0} \geq 2$. We also know from Lemma A.7 that there is a category $g_{0} \leq h_{0}$ satisfying $g_{0} \geq 2$ for which we have:

$$
\sum_{h=1}^{g_{0}} \bar{p}^{\mathbf{y}}\left(h, y_{1}^{1}\right) \leq \sum_{h=1}^{g_{0}} \bar{p}^{\mathbf{x}}\left(h, y_{1}^{1}\right),
$$

and

$$
\sum_{h=1}^{l} \bar{p}^{\mathbf{y}}\left(h, y_{1}^{1}\right)>\sum_{h=1}^{l} \bar{p}^{\mathbf{x}}\left(h, y_{1}^{1}\right)
$$

for all $l=1, \ldots, g_{0}-1$. As a consequence we have:

$$
\sum_{h=l+1}^{g_{0}} \bar{p}^{\mathbf{y}}\left(h, y_{1}^{1}\right)<\sum_{h=l+1}^{g_{0}} \bar{p}^{\mathbf{x}}\left(h, y_{1}^{1}\right)
$$


for $l=1, \ldots, g_{0}-1$. and the conclusion of the proposition follows from Lemma A.3.

A.7 Proof of the Finiteness of the Algorithm of Subsection 3.2. Suppose by contradiction that the algorithm defined in Subsection 3.2 generates an infinite sequence $(\mathbf{x}(n))_{n \in \mathbb{N}}$. We proceed by first establishing the following claims.

Claim A.1. There exists some $n_{0} \in \mathbb{N}$ such that, for all $n \geq n_{0}$, either case (P2) or $(M T)$ of Subsection 3.2 holds, so that $x_{1}^{1}(n)<v_{1}^{c}(n)$.

Proof. Once an agent of a category higher than 1 has been involved in an FIP defined as in Proposition 3, his/her income becomes weakly smaller than that of the poorest income observed in category 1 . Since the income of an agent in any category $h \geq 2$ never increases through the algorithm described above, and since the number of agents in categories higher than 1 is finite, it follows that the the number of FIP of type $(P 1)$ in the algorithm is bounded above by the number of agents in categories $2, \ldots, k$.

An immediate consequence of this claim is that, for any $n \geq n_{0}$, we can also define the quantities $v_{h}^{c}(n)$ through expression (10).

Claim A.2. Let $n_{0}$ be the integer whose existence was established in Claim A.1. Then, for all $n \geq n_{0}$ and all categories $h=1, \ldots, k$, we have $v_{h}^{c}(n+1) \leq v_{h}^{c}(n)$.

Proof. By definition of the algorithm and the critical vector $\mathbf{v}^{\mathbf{c}}(\mathbf{n})$, we have $P^{\mathbf{x}(n)}\left(\mathbf{v}^{\mathbf{c}}(\mathbf{n})\right)=$ $P^{\mathbf{x}}\left(\mathbf{v}^{\mathbf{c}}(\mathbf{n})\right)$ which directly implies that:

$$
P^{\mathbf{x}(n)}\left(\mathbf{v}^{\mathbf{c}}(\mathbf{n})\right)=P^{\mathbf{x}(n+1)}\left(\mathbf{v}^{\mathbf{c}}(\mathbf{n})\right)=P^{\mathbf{x}}\left(\mathbf{v}^{\mathbf{c}}(\mathbf{n})\right) .
$$

We first observe that if the distribution $\mathbf{x}(n+1)$ is obtained from $\mathbf{x}(n)$ through a maximal transfer (MT), the donor's income is equal to $v_{1}^{c}(n)$ and therefore the recipient being the poorest agent in category 1 , we necessarily have $x_{1}^{1}(n+1)<v_{1}^{c}(n)$. On the other hand, if distribution $\mathbf{x}(n+1)$ is obtained from $\mathbf{x}(n)$ through an FIP of type $(P 2)$, the recipient has an income equal to $v_{1}^{c}(n)>x_{1}^{1}(n)$, so that $x_{1}^{1}(n)=x_{1}^{1}(n+1)<v_{1}^{c}(n)$. Hence, in either case, we have $x_{1}^{1}(n+1)<v_{1}^{c}(n)$. Now by definition of $v_{1}^{c}(n+1)$ as an infimum, identity (A.18) and the fact that $x_{1}^{1}(n+1)<v_{1}^{c}(n)$, we have $v_{1}^{c}(n+1) \leq v_{1}^{c}(n)$. Now combining $P^{\mathbf{x}}\left(v^{c}(n)\right)=$ $P^{\mathbf{x}(n+1)}\left(v^{c}(n)\right)$ and $P^{\mathbf{x}}\left(v^{c}(n+1)\right)=P^{\mathbf{x}(n+1)}\left(v^{c}(n+1)\right)$ on the one hand and Corollary A.1 on the other, it follows that $v_{h}^{c}(n+1) \leq v_{h}^{c}(n)$ holds for all $h$ as well.

In the next claim, we establish the existence of some step in the algorithm beyond which no FIP occurs.

Claim A.3. There exists $n_{1} \in \mathbb{N}$ such that, for any $n \geq n_{1}$, the distribution $\mathbf{x}(n+1)$ is obtained from $\mathbf{x}(n)$ by means of a maximal transfer.

Proof. We first observe that $\bar{p}^{\mathbf{x}(n)}\left(1, v_{1}^{c}(n)\right)$ is weakly decreasing for $n \geq n_{0}$, where $n_{0}$ is the integer whose existence was established in Claim A.1. Indeed $v_{1}^{c}(n)$ is weakly decreasing for $n \geq n_{0}$ and an agent in category 1 can be designated as the donor at step $n$ only if the algorithm prescribes a maximal transfer and his/her income is equal to $v_{1}^{c}(n)$. This proves that the number of agents in category 1 of distribution $\mathbf{x}(n)$ whose income is weakly smaller than $v_{1}^{c}(n)$ necessarily weakly decreases as $n$ increases.

Assume now that at some stage $n \geq n_{0}$ we are in case $(\mathrm{P} 2)$. In that case, the receiving agent's income is equal to $v_{1}^{c}(n)$. Hence $\bar{p}^{\mathbf{x}(n+1)}\left(1, v_{1}^{c}(n+1)\right)<\bar{p}^{\mathbf{x}(n)}\left(v_{1}^{c}(n)\right)$. As a result, there can be at most $n(1)$ operations of type (P2) in the algorithm after step $n_{0}$. 
We proved that, for any $n \geq n_{1}$, a maximal transfer of type $(M T)$ occurs at time $n$. Since the algorithm is infinite, no transfer can be equalizing as per Definition 6. Hence, the maximal transfers at every step must be either a breaking or a half transfer of Definition 6 . We next claim that at every step after $n_{1}$, if a breaking transfer is required by the algorithm, then the donor involved in the transfer will never be the donor again in a subsequent transfer. Although the proof of the claim is slightly cumbersome, the intuition behind it is relatively clear. Indeed, by its very definition, a breaking transfer is such that the donor cannot give more at this stage without breaking at least one of the OPG dominance inequalities. As $n$ increases, the (OPG) difference between distribution $\mathbf{x}(n)$ and distribution $\mathbf{x}$ gets smaller and smaller. Hence it becomes harder and harder to make a transfer without breaking some of the OPG inequalities.

Claim A.4. There exists $n_{2} \in \mathbb{N}$ such that, for any $n \geq n_{2}$, distribution $\mathbf{x}(n+1)$ is obtained from $\mathbf{x}(n)$ through a half transfer.

Proof. Let $n_{1}$ be as in Claim A.3. We need to establish that there can only be finitely many breaking transfers after stage $n_{1}$. Consider any $n \geq n_{1}$ and suppose that $\mathbf{x}(n+1)$ is obtained from $\mathbf{x}(n)$ through a breaking transfer of amount $\alpha>0$ from agent $j_{h} \in \mathcal{N}(h)$ (with $h \geq 1$ ) to agent $1 \in \mathcal{N}(1)$. Let $r_{+}^{1}(\alpha) \in \mathcal{N}(1)$ and $r_{-}^{h}(\alpha) \in \mathcal{N}(h)$ be as in Definition 1. Hence, $x_{r_{+}^{1}(\alpha)}^{1}(n+$ $1)=x_{1}^{1}(n)+\alpha$ and $x_{r_{-}^{h}(\alpha)}^{h}(n+1)=x_{j_{h}}^{h}(n)-\alpha$.

Let $\delta>0$. By definition of a breaking transfer, there exists $\mathbf{v}(\delta) \in \mathcal{V}^{\prime}$ such that:

$$
P^{\mathbf{x}(n+1)^{\delta}}(\mathbf{v}(\delta))<P^{\mathbf{x}}(\mathbf{v}(\delta))
$$

where $\mathbf{x}(n+1)^{\delta}$ denotes the distribution that would be obtained if the transfer at time $n$ was equal to $\alpha+\delta$ instead of $\alpha$. By compactness of $\mathcal{V}^{\prime}$, we may assume without loss of generality that $\lim _{\delta \rightarrow 0} \mathbf{v}(\delta)=\mathbf{v}^{*} \in \mathcal{V}^{\prime}$. By continuity, we then have $P^{\mathbf{x}(n+1)}\left(\mathbf{v}^{*}\right)=P^{\mathbf{x}}\left(\mathbf{v}^{*}\right)$. Note that without loss of generality, we can assume that $v_{1}(\delta) \geq x_{1}^{1}(n)+\alpha$. This implies that $v_{1}^{*} \geq x_{1}^{1}(n)+\alpha$.

We now show that $v_{1}^{*}>x_{1}^{1}(n+1)$. By contradiction assume that $v_{1}^{*} \leq x_{1}^{1}(n+1)$. Then, since $x_{1}^{1}(n+1) \leq x_{1}^{1}(n)+\alpha$, we necessarily have $v_{1}^{*}=x_{1}^{1}(n)+\alpha=x_{1}^{1}(n+1)$, that is, the poorest agent in category 1 remains the poorest agent after receiving $\alpha$ at step $n$. Thus at next step (step $n+1)$ the algorithm identifies him as the recipient again. Let $h^{\prime} \geq 1$ and $j_{h^{\prime}} \in \mathcal{N}\left(h^{\prime}\right)$ be the donor at next step $n+1$ and suppose he/she transfers $\delta>0$. Since $v_{h^{\prime}}^{*} \leq v_{1}^{*}=x_{1}^{1}(n+1)<$ $x_{j_{h^{\prime}}}^{h^{\prime}}(n+1)$, we have:

$$
P^{\mathbf{x}(n+2)}(\mathbf{v}(\delta))=P^{\mathbf{x}(n+1)^{\delta}}(\mathbf{v}(\delta))<P^{\mathbf{x}}(\mathbf{v}(\delta)),
$$

a contradiction. Since $v_{1}^{*}>x_{1}^{1}(n+1)$, we must have $v_{h}^{c}(n+1) \leq v_{h}^{*}$ for any $h$ by Corollary A.1. By Claim 2, this implies that $v_{h}^{c}(m) \leq v_{h}^{*}$ for any $m \geq n+1$.

We now claim that for $m>n$, if the donor at step $m$ is in category $h$, his/her income can not be equal to $x_{r_{-}^{h}(\alpha)}^{h}(n+1)$. Given the finiteness of the population, this will conclude the proof, because it will exclude the donor at stage $n$ from donating again at a future step. Suppose, to the contrary, that there exists $m \geq n+1$ and $l_{h} \in \mathcal{N}(h)$ such that $v_{1}^{c}(m)=v_{h}^{c}(m)=x_{l_{h}}^{h}(m)=$ $x_{r_{-}^{h}}^{h}(n+1)$, and that agent $l_{h}$ transfers $\delta_{0}>0$ to agent $1 \in \mathcal{N}(1)$ at stage $m$. We then have:

$$
x_{1}^{1}(m)<x_{l_{h}}^{h}(m)=x_{r_{-}^{h}(\alpha)}^{h}(n+1)=v_{1}^{c}(m) \leq v_{1}^{*} .
$$

Assume without loss of generality that $\delta_{0}$ is small enough so that $x_{1}^{1}(m) \leq v_{1}\left(\delta_{0}\right)-\delta_{0}$. Then:

$$
P^{\mathbf{x}(m+1)}\left(\mathbf{v}\left(\delta_{0}\right)\right)-P^{\mathbf{x}(m)}\left(\mathbf{v}\left(\delta_{0}\right)\right)=P^{\mathbf{x}(n+1)^{\delta_{0}}}\left(\mathbf{v}\left(\delta_{0}\right)\right)-P^{\mathbf{x}(n+1)}\left(\mathbf{v}\left(\delta_{0}\right)\right)
$$


(both quantities are equal to $-\delta_{0}+\max \left\{0, v_{h}\left(\delta_{0}\right)-\left(x_{r_{-}^{h}(\alpha)}^{h}(n+1)-\delta_{0}\right)\right\}-\max \left\{0, v_{h}\left(\delta_{0}\right)-\right.$ $\left.\left.x_{\left.r_{-}^{h}(\alpha)\right)}^{h}(n+1)\right\}\right)$. Since $P^{\mathbf{x}(m)}\left(\mathbf{v}\left(\delta_{0}\right)\right) \leq P^{\mathbf{x}(n+1)}\left(\mathbf{v}\left(\delta_{0}\right)\right)$, we have

$$
P^{\mathbf{x}(m+1)}\left(\mathbf{v}\left(\delta_{0}\right)\right) \leq P^{\mathbf{x}(n+1)^{\delta_{0}}}\left(\mathbf{v}\left(\delta_{0}\right)\right)<P^{\mathbf{x}}\left(v\left(\delta_{0}\right)\right)
$$

a contradiction.

We now establish that none of the donors involved in the half transfers that remain after all breaking transfers have been performed can be in category 1 .

Claim A.5. For any $n \geq n_{2}$, the distribution $\mathbf{x}(n+1)$ is obtained from $\mathbf{x}(n)$ through a half transfer whose donor is not in category 1.

Proof. Let $n \geq n_{2}$. We proved already that the operation at stage $n$ is necessarily a half transfer. Let $h_{0}$ be the category such that $v_{1}^{c}(n)=v_{h_{0}}^{c}(n)>v_{h_{0}+1}^{c}(n)$. Suppose by contradiction that the algorithm designates $i \in \mathcal{N}(1)$ to be the donor at stage $n$. By Proposition 1, it implies that $\forall h \in\left\{2, \ldots, h_{0}\right\}, \forall i \in \mathcal{N}(h)$, we must have $x_{i}^{h}(n) \neq v_{1}^{c}(n)$ because, otherwise, an agent in category $h>1$ would be the donor. Note also that, by the very definition of a maximal transfer, we must have $x_{i}^{1}>v_{1}^{c}(n)$ for any $i \in \mathcal{N}(1)$ because assuming otherwise would make the transfer equalizing, which it can not be. Consequently the conditions of (P2) hold, which is a contradiction.

We are now ready to complete the proof that our algorithm is indeed finite. By Claim A.5, if the algorithm is infinite, there is some $n_{2} \in \mathbb{N}$ such that, for $n \geq n_{2}, \mathbf{x}(n+1)$ is obtained from $\mathbf{x}(n)$ through a half transfer, the donor of which is not in category 1 . Yet, once an agent in category 1 has received a half transfer from an agent of a higher category, his/her income becomes equal to that of the donating agent. Hence, the donating agent cannot be selected again by the algorithm to donate to that same receiving agent. Since the number of agents is finite, this completes the proof.

Proof of Theorem 2. (i) $\Rightarrow$ (ii) follows from the proof in the homogeneous settings, along with the fact that $U^{h^{\prime}} \geq U^{h}$ for $h^{\prime} \geq h$ to handle the categorical increments.

(ii) $\Rightarrow$ (iii) the proof of the corresponding implication in Subsection A.2 can be applied here without any change.

$($ iii $) \Rightarrow($ i) This implication is not trivial. Suppose that (iii) holds:

$$
\Delta(\mathbf{v}) \leq 0 \forall \mathbf{v} \in \mathcal{V}
$$

where $\Delta(\mathbf{v}):=\sum_{h=1}^{k} \sum_{i \in \mathcal{N}_{\mathbf{x}}(h)} \max \left(v_{h}-x_{i}^{h}, 0\right)-\sum_{h=1}^{k} \sum_{i \in \mathcal{N}_{\mathbf{y}}(h)} \max \left(v_{h}-y_{i}^{h}, 0\right)$. Then we claim that

$$
\sum_{h=1}^{g} n_{\mathbf{y}}(h) \geq \sum_{h=1}^{g} n_{\mathbf{x}}(h), \quad \forall g=1, \ldots, k
$$

Let us prove this claim. Suppose that there exists some $g^{*}$ such that $\sum_{h=1}^{g^{*}} n_{\mathbf{y}}(h)<\sum_{h=1}^{g^{*}} n_{\mathbf{x}}(h)$. For any $\alpha \geq \bar{v}(\mathbf{x}, \mathbf{y})$ let $\mathbf{v}(\alpha) \in \mathcal{V}$ be given by $v(\alpha)_{h}:=\underline{v}(\mathbf{x}, \mathbf{y})$ for $h=g^{*}+1, \ldots, k$ and $v(\alpha)_{h}=$ $\alpha$ for $h=1, \ldots, g^{*}$. We then have

$$
\sum_{h=1}^{k} \sum_{i=1}^{n_{\mathbf{x}}(h)} \max \left(v(\alpha)_{h}-x_{i}^{h}, 0\right)=\sum_{h=1}^{g^{*}} \sum_{i=1}^{n_{\mathbf{x}}(h)}\left(\alpha-x_{i}^{h}\right)=\alpha \sum_{h=1}^{g^{*}} n_{\mathbf{x}}(h)-\sum_{h=1}^{g^{*}} \sum_{i=1}^{n_{\mathbf{x}}(h)} x_{i}^{h} .
$$


As a consequence

$$
\Delta(\mathbf{v}(\alpha))=\alpha\left(\sum_{h=1}^{g^{*}} n_{\mathbf{x}}(h)-\sum_{h=1}^{g^{*}} n_{\mathbf{y}}(h)\right)+\sum_{h=1}^{g^{*}}\left(\sum_{i=1}^{n_{\mathbf{y}}(h)} y_{i}^{h}-\sum_{i=1}^{n_{\mathbf{x}}(h)} x_{i}^{h}\right) .
$$

As a goes to $+\infty$, the right-hand side term goes to $+\infty$, which contradicts (iii), and proves the claim.

If $\sum_{h=1}^{g} n_{\mathbf{y}}(h)=\sum_{h=1}^{g} n_{\mathbf{x}}(h), \forall g=1, \ldots, k$ then there is nothing to prove. Let us assume that this is not the case and let $h^{*} \in\{1, \ldots, k-1\}$ be the lowest category such that $\sum_{h=1}^{g} n_{\mathbf{y}}(h)>$ $\sum_{h=1}^{g} n_{\mathbf{x}}(h)$. Let $\mathbf{y}$ be the distribution obtained from $\mathbf{y}$ through an increment of agent $y_{n_{\mathbf{y}}\left(h^{*}\right)}$ to category $h^{*}+1$. We claim that $\overline{\mathbf{y}} \precsim O P G \mathbf{x}$. This will conclude the proof by induction. Let $\mathbf{v} \in \mathcal{V}$. If $v_{h^{*}} \leq y_{n_{\mathbf{y}}\left(h^{*}\right)}^{h^{*}}$ then $P^{\mathbf{x}}(\mathbf{v})-P^{\overline{\mathbf{y}}}(\tilde{\mathbf{v}})=P^{\mathbf{x}}(\mathbf{v})-P^{\mathbf{y}}(\mathbf{v}) \leq 0$. If $v_{h^{*}}>y_{n_{\mathbf{y}}\left(h^{*}\right)}^{h^{*}}$ then

$$
P^{\mathbf{y}}(\mathbf{v})-P^{\overline{\mathbf{y}}}(\mathbf{v})=v_{h^{*}}-y_{n_{\mathbf{y}}\left(h^{*}\right)}^{h^{*}}-\max \left\{0, v_{h^{*}+1}-y_{n_{\mathbf{y}}\left(h^{*}\right)}^{h^{*}}\right\}
$$

On the other hand, let $\tilde{\mathbf{v}}:=\left(v_{1}, \ldots, \max \left\{y_{n_{\mathbf{y}}\left(h^{*}\right)}^{h^{*}}, v_{h^{*}+1}\right\}, v_{h^{*}+1}, \ldots v_{k}\right)$. Clearly $\tilde{\mathbf{v}} \in \mathcal{V}$ and

$$
\begin{aligned}
P^{\mathbf{y}}(\mathbf{v})-P^{\mathbf{x}}(\mathbf{v}) & \geq P^{\mathbf{y}}(\tilde{\mathbf{v}})-P^{\mathbf{x}}(\tilde{\mathbf{v}})+\left(n_{\mathbf{y}}\left(h^{*}\right)-n_{\mathbf{x}}\left(h^{*}\right)\right)\left(v_{h^{*}}-\max \left\{y_{n_{\mathbf{y}}\left(h^{*}\right)}^{h^{*}}, v_{h^{*}+1}\right\}\right) \\
& \geq v_{h^{*}}-\max \left\{y_{n_{\mathbf{y}}\left(h^{*}\right)}^{h^{*}}, v_{h^{*}+1}\right\} \\
& =v_{h^{*}}-y_{n_{\mathbf{y}}\left(h^{*}\right)}^{h^{*}}-\max \left\{0, v_{h^{*}+1}-y_{n_{\mathbf{y}}\left(h^{*}\right)}^{h^{*}}\right\} \\
& =P^{\mathbf{y}}(\mathbf{v})-P^{\overline{\mathbf{y}}}(\mathbf{v}) .
\end{aligned}
$$

Consequently $P^{\overline{\mathbf{y}}}(\mathbf{v}) \geq P^{\mathbf{x}}(\mathbf{v})$, which concludes the proof.

$($ iii $) \Leftrightarrow($ iv $)$ : the proof of the corresponding implication in Subsection 3.2 can be applied here without any change.

\section{REFERENCES}

Anderson, G., "Nonparametric Tests of Stochastic Dominance in Income Distributions," Econometrica 64 (1996), 1183-93.

AtKinson, A., "On the Measurement of Poverty," Econometrica 55 (1987), 749-64.

Atrinson, A. B., And F. Bourguignon, "The Comparison of Multi-dimensioned Distribution of Economic Status," Review of Economic Studies 49 (1982), 183-201.

- "Income Distributions, and Differences in Needs," in G. R. Feiwel, ed., Arrow and the Foundation of the Theory of Economic Policy (London: Macmillan, 1987).

Beach, C. M., AND R. Davidson, "Distribution-Free Statistical Inference with Lorenz Curves and Income Shares," Review of Economic Studies 50 (1983), 723-35.

Berge, C., Espaces topologiques et fonctions multivoques (Paris: Dunod, 1959).

Bishop, C. M., S. Chakraborti, and P. Thistle, "Asymptotically Distribution-Free Statistical Inference for Generalized Lorenz Curves," Review of Economics and Statistics 71 (1989a), 725-27.

—, AND J. P. Formby, "Test of Significance for Lorenz Partial Orders," in J. Silber, ed., Handbook of Inequality Measurement (Kluwer Academic Press, 1999).

$\longrightarrow,-, \ldots$ AND W. SMITH, "International Comparisons of Income Inequality:Tests for Lorenz Dominance across Nine Countries," Economica 58 (1989b), 461-77.

Bourguignon, F., "Family Size and Social Utility: Income Distribution Dominance Criteria," Journal of Econometrics 42 (1989), 67-80.

Chakravarty, S., and P. Muliere, "Welfare Indicators: A Review and New Perspectives," Metron 61 (2003), 457-97.

Cowell, F. A., Measuring Inequality, 3rd edition (Oxford: Oxford University Press, 2011).

Dalton, H., "The Measurement of the Inequality of Incomes," Economic Journal 30 (1920), 348-61. 
Dasgupta, P., A. K. Sen, and D. Starrett, "Notes on the Measurement of Inequality," Journal of Economic Theory 6 (1973), 180-87.

Davidson, R., AND J. Y. Duclos, "Statistical Inference for Stochastic Dominance and for the Measurement of Poverty and Inequality," Econometrica 58 (2000), 1435-65.

DecancQ, K., "Elementary Multivariate Frechet Rearrangements and Stochastic Dominance on a Frechet Class," Journal of Economic Theory 147 (2012), 1450-59.

Decoster, A., And E. Ooghe, "A Bounded Index Test to Make Robust Heterogenous Welfare Comparisons," Review of Income and Wealth 52 (2006), 361-76.

Duclos, P. Y., D. Sahn, and S. D. Younger, "Robust Multidimensional Poverty Comparisons," The Economic Journal 116 (2006), 943-68.

Eвert, U., "Social Welfare When Needs Differ: An Axiomatic Approach," Economica 64 (1997), 23344.

Epstein, L., And S. M. TAnny, "Increasing Generalized Correlation: A Definition and Some Economic Consequences," Canadian Journal of Economics 13 (1980), 16-34.

Fields, G., AND J. FeI, “On Inequality Comparisons,” Econometrica 46 (1978), 305-16.

Fleurbaey, M., C. Hagneré, and A. Trannoy, "Welfare Comparisons with Bounded Equivalence Scales," Journal of Economic Theory 110 (2003), 309-36.

Foster, J., "Inequality Measurement," in H. P. Young, ed., Fair Allocation (Providence, RI: American Mathematical Society, 1985), 38-61.

Gravel, N., And P. Moyes, "Ethically Robust Comparisons of Bi-dimensionnal Distributions with an Ordinal Attribute," Journal of Economic Theory 147 (2012), 1384-1426.

_, "Utilitarianism or Welfarism: Does It Make a Difference?," Social Choice and Welfare 40 (2013), 529-51.

,-- AND B. TARroux, "Robust International Comparisons of Distributions of Disposable Income and Access to Regional Public Goods," Economica 76 (2009), 432-61.

mensional Answer," Journal of Economic Inequality 8 (2010), 173-95.

Hadar, J., ANd W. Russell, "Stochastic Dominance in Choice under Uncertainty," in M. S. Balch, D. L. McFadden and S. Wu, eds., Essays on Economics Behavior under Uncertainty (Amsterdam, UK: North Holland, 1974).

Hardy, G. H., J. E. LitTlewood, and G. Polya, Inequalities, 2nd edition (Cambridge, UK: Cambridge University Press, 1952).

Kolm, S. C., "The Optimal Production of Social Justice," in H. Guitton and J. Margolis, eds.,Public Economics (London: Macmillan, 1969).

_ , "Multidimensional Egalitarianisms," Quarterly Journal of Economics 91 (1977), 1-13.

Koshevoy, G., "Multivariate Lorenz Majorization," Social Choice and Welfare 12 (1995), 93-102.

Levhari, D., J. Paroush, and B. Peleg, "Efficiency Analysis for Multivariate Distributions," Review of Economic Studies 42 (1975), 87-91.

Marinacci, M., And L. Montrucchio, "Ultramodular Functions," Mathematics of Operation Research 30 (2005), 311-32.

Moyes, P., "Comparaisons of Heterogeneous Distributions and Dominance Criteria," Journal of Economic Theory 147 (2012), 1351-83.

Muller, A., AND M. SCARsini, "Fear of Loss, Inframodularity and Transfers," Journal of Economic Theory 147 (2012), 1490-1500.

Osterdal, L. P., "The Mass Transfer Approach to Multivariate Discrete First Order Stochastic Dominance: Direct Proof and Implications," Mathematical Social Sciences 46 (2010), 1222-28.

Quirk, J. D., AND R. SAPOSNiK, "Admissibility and Measurable Utility Functions," Review of Economic Studies 29 (1962), 140-46.

SEN, A. K., On Economic Inequality (Oxford: Clarendon, 1973).

Shorrocks, A. F., "Ranking Income Distributions," Economica 50 (1983), 3-17.

Tchen, A. H., "Inequalities for Distributions with Given Marginals," Annals of Probability 8 (1980), 814-27.

Tsui, K. Y., "Multidimensional Inequality and Multidimensionnal Generalized Entropy Measures: An Axiomatic Derivation," Social Choice and Welfare 16 (1999), 145-57.

Zheng, B., "Unit Consistent Poverty Indices," Economic Theory 31 (2007), 113-42. 